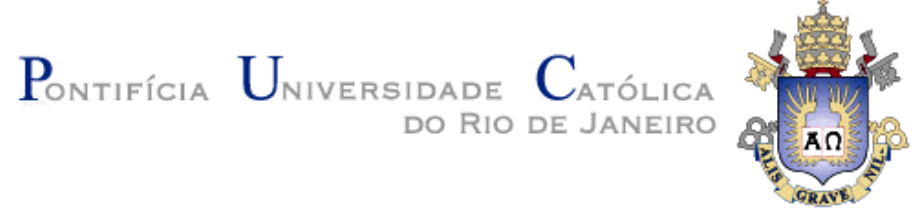

Sergio Gutiérrez Escobar

\title{
Humidity sensor based on MEMS SAW Technology
}

\section{Dissertação de Mestrado}

Dissertation presented to the Programa de Pósgraduação em Engenharia Mecânica of PUC-Rio in partial fulfillment of the requirements for the degree of Mestre em Engenharia Mecânica.

Advisor: Prof. Arthur Martins Barbosa Braga

Co-Advisor: Dr. Sully Milena Mejia Quintero

Rio de Janeiro

September 2016. 
Sergio Gutiérrez Escobar

\section{Humidity sensor based on MEMS SAW Technology}

Dissertation presented to the Programa de Pós-graduação em Engenharia Mecânica of PUC-Rio in partial fulfillment of the requirements for the degree of Mestre em Engenharia Mecânica.

Approved by the undersigned Examination Committee.

Prof. Arthur Martins Barbosa Braga

Advisor

Departamento de Engenharia Mecânica- PUC-Rio

Dr. Sully Milena Mejia Quintero

Co- Advisor

Departamento de Engenharia Mecânica- PUC-Rio

Dr. Serguei Balachov

Researcher CTI Renato Archer

Dr. Manoel Feliciano da Silva Junior

Cenpes/Petrobras

Prof. Márcio da Silveira Carvalho

Vice Dean of Graduate Studies

Centro Técnico Científico - PUC-Rio

Rio de Janeiro, September 13th, 2016 
All rights reserved

Sergio Gutiérrez Escobar

Graduated in Mechanical Engineering from Universidad Industrial de Santander (UIS), Bucaramanga - Colombia in 2013. Currently pursuing a master's degree in mechanical engineering from PUC-Rio.

Bibliographic data

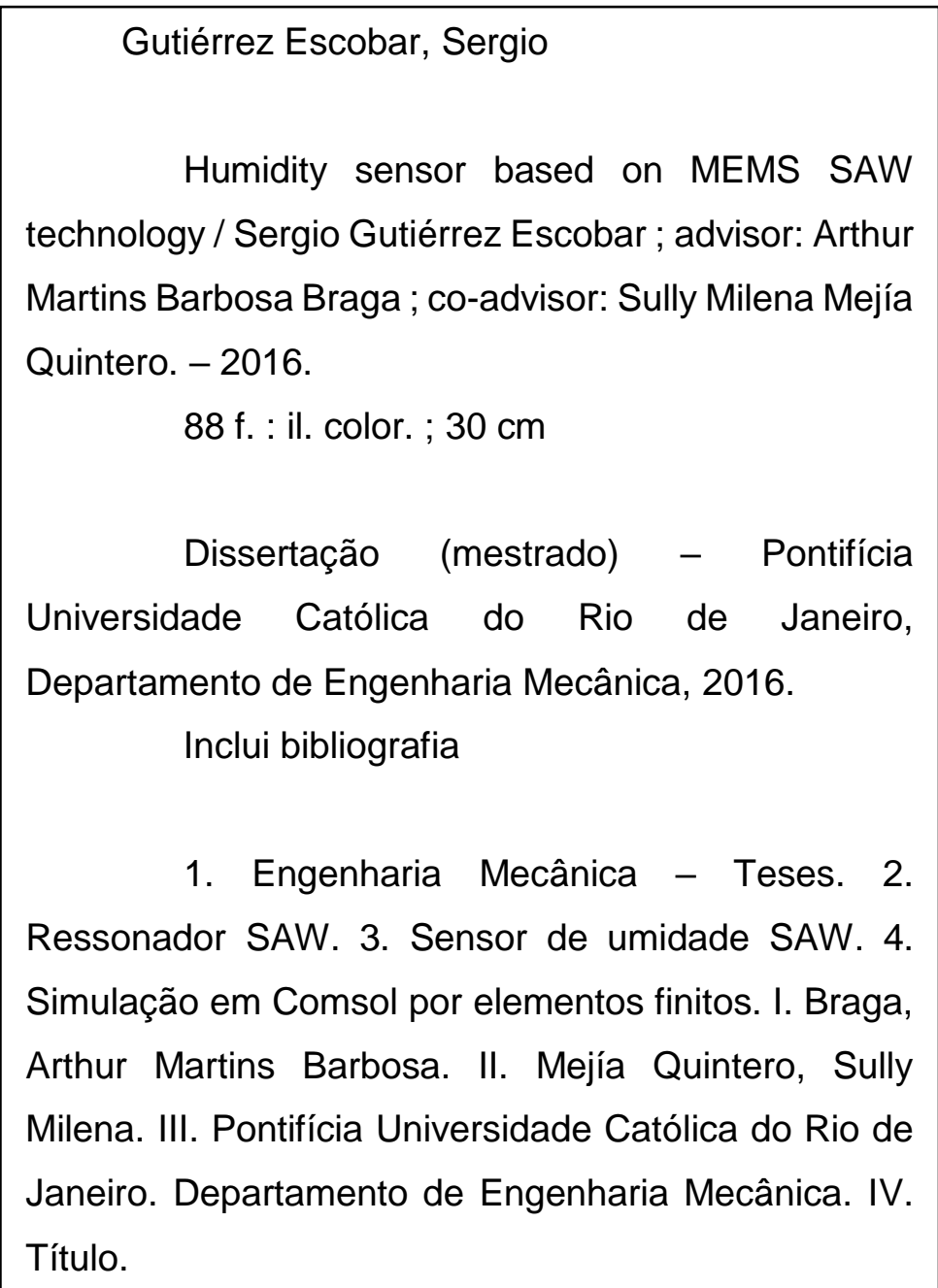




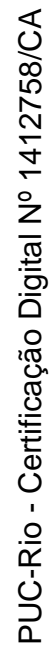

I dedicate the Dissertation to my parents Luis Alfonso Gutiérrez and Martha Patricia Escobar 


\section{Acknowledgments}

To God for his guide, support, strength and blessing through my life.

To my parents, Martha Escobar and Luis A. Gutierrez for all their love, dedication, support and guidance.

To my brothers Renato and Alfonso Gutierrez Escobar for all their support, help and love.

To my Girlfriend Hayane Maciel for her patience, support, love and to believe in me.

To my advisors, Arthur Braga for his support and help, to Sully Mejia for her support, guidance, patience, dedication, help and friendship, this achievement is also yours. To both, the knowledge given.

To my colleagues of the LSFO, Victor, Savio, André, Guilherme, Rafael, and the rest of the team for their help.

To all the team of the Cremona's Lab for their help and patience.

To all colleagues of DEM PUC-Rio.

To the ANP for their support. 


\section{Abstract}

Gutiérrez Escobar, Sergio; Braga, Arthur Martins Barbosa (Advisor); Mejía Quintero, Sully Milena (Co-advisor). Humidity sensor based on MEMS

SAW Technology. Rio de Janeiro, 2016. 88p. MSc. DissertationDepartamento de Engenharia Mecânica, Pontifícia Universidade Católica do Rio de Janeiro.

Micro electromechanical systems (MEMS) are devices that combine mechanical structures with electrical circuits at the micro scale, to function as sensors or actuators. One type of MEMS are the surface acoustic waves (SAW) devices, which uses the surface wave velocity or propagation path variations to measure the variable of interest. One important application in chemical processes is related to environment condition control, specifically humidity measurement. With that purpose, a commercial SAW was purchased and coated with a polymer layer in its surface. The PolyVynil Alcohol (PVA) was chosen to be the sensing layer in the SAW due to water vapor absorption properties, that increases the mass over the surface and decrease the wave velocity, leading to sense this humidity changes. 5.6-wt \% PVA solution was prepared and deposited through spin coating. Therefore, a series of tests were carried out in a climatic chamber, varying the humidity and temperature conditions, with the aim to analyze the sensor behavior by measuring its frequency shift. These results were compared with an analytical model and a finite element simulation. The analytical model presented by Sielman determines how the polymer density changes with humidity. These density values were inserted into the Wohltjen equation, which gives the frequency shift of the SAW due to gas absorption. Regarding the finite element simulation, it was carried out in the Comsol Multiphysics software, by solving the different resonating frequencies as a function of the increase in the polymer density due to the insets of humidity values.

\section{Keywords}

SAW Resonator; Humidity SAW sensor; Comsol SAW Model, 


\section{Resumo}

Gutiérrez Escobar, Sergio; Braga, Arthur Martins Barbosa; Mejía Quintero, Sully Milena. Sensor de umidade Baseado em Tecnologia MEMS SAW. Rio de Janeiro, 2016. 88p. Dissertação de Mestrado - Departamento de Engenharia Mecânica, Pontifícia Universidade Católica do Rio de Janeiro.

Os sistemas micro eletromecânicos são dispositivos na escala dos micras que combinam estruturas mecânicas com circuitos elétricos, e são usados como sensores ou atuadores. Dentro destes dispositivos, estão os de onda superficial acústica (SAW em inglês) que usam variações na velocidade ou percurso de propagação da onda para fazer a detecção da variável a medir. Uma aplicação importante em processos químicos, é no acondicionamento de ambientes, monitorando a umidade. Para isso um sensor SAW comprado, foi coberto em sua superfície com uma camada de um polímero absorvente de vapor de agua. No qual o aumento na massa do polímero na superfície diminui a velocidade da onda. Por tanto o PolyVinyl Álcool foi escolhido para absorver o vapor de agua e foi preparado com $5.6 \mathrm{wt} \%$, para ser depositado por meio de spin coating. Então uma serie de experimentos foram feitos numa câmara climática variando tanto a umidade como a temperatura, com o fim de avaliar o comportamento do sensor medindo a sua variação da frequência. Estes resultados foram comparados com um modelo analítico e uma simulação por elementos finitos. O modelo analítico foi presentado por Sielman, o qual determina como muda a densidade e espessura no polímero com a umidade. Estes valores foram substituídos na equação de Wohltjen que dá a variação da frequência de um SAW devido a absorção de gases. Em quanto a simulação por elementos finitos foi feita em Comsol Multiphysics achando a frequência para a qual o SAW ressona, com o aumento da densidade na camada acima do SAW para as umidades inseridas.

\section{Palavras-chave}

Ressonador SAW; Sensor de umidade SAW; Modelo SAW em Comsol. 


\section{Contents}

1 Introduction 13

1.1 Problem definition 13

1.2 Motivation and Objectives 13

1.3 Dissertation outline 14

2 MEMS Technology 15

2.1 MEMS fabrication process 17

2.1.1 Deposition methods: 18

2.1.1.1 Epitaxy: 18

2.1.1.2 Oxidation: $\quad 18$

2.1.1.3 Sputtering: $\quad 19$

2.1.1.4 Evaporation: 20

2.1.1.5 Chemical Vapor Deposition (CVD): $\quad 20$

2.1.2 Patterning: 21

2.1.2.1 Photolithography: 21

2.1.3 Etching: 21

2.1.3.1 Dry Or Wet etching: 22

2.2 MEMS sensing principle 23

2.2.1 Piezoelectric MEMS: 23

2.2.2 Piezoresistive MEMS: 23

2.2.3 Capacitive MEMS: 24

2.2.4 Examples of MEMS sensors: 25

2.3 MEMS Packaging 27

2.3.1 Zero level package $\quad 27$

2.3.2 First Level packaging: $\quad 28$

2.3.3 Packaging types 29

2.4 Application to Humidity measurements 31

3 SAW Technology 35

3.1 Physics of surface acoustic waves devices 40

3.1.1 Acoustic waves 40

3.1.1.1 Wave equation 42

3.1.2 The Piezo electricity effect 44

3.1.3 Piezoelectric crystals 45

3.1.3.1 Crystal structure $\quad 45$

3.1.3.2 Crystal cuts 49

3.2 Wave modes 51 
4 Experiments 56

4.1 Sensing Polymer 56

4.1.1 Diffusion and Fick's Law 57

4.1.2 Preparation of Poly(vinyl Alcohol) Films 58

4.1.3 PVA deposition 58

4.1.4 PVA Film mechanical properties characterization 59

4.1.5 Measurement system diagram of the for the humidity sensor based on SAW 62

4.2 Wired Interrogation System for Saw Sensors 63

4.3 Experimental setup 64

4.4 Methodology and Results 65

4.4.1 Test 1: $\quad 66$

4.4.2 Test 2: $\quad 67$

4.4.3 Test 3: $\quad 68$

4.4.4 Teste 4- 5: $\quad 69$

4.5 Analytical SAW Mass-only Response $\quad 70$

4.5.1 Partial density Method: 72

5 Finite Element model for SAW devices $\quad 74$

5.1 Finite Element Analysis (FEA) for SAW Devices 74

5.1.1 Survey of important characteristics of the SAW and coating film 74

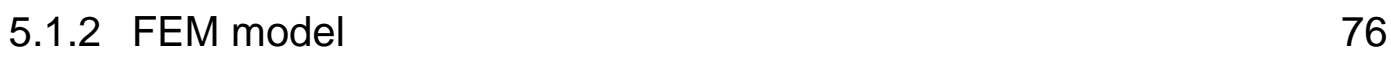

5.1.3 Simulation Results 77

6 Conclusions and Future Work 81

7 Bibliography 83

A SAW data sheet $\quad 88$ 


\section{List of Figures}

Figure 2.1 Schematic structure of MEMS 16

Figure 2.2 History of MEMS 16

Figure 2.3 Typical MEMS fabrication process 18

Figure 2.4 Epitaxy process scheme 18

Figure 2.5 Oxidation chamber 19

Figure 2.6 Sputtering process 19

Figure 2.7 Evaporation process scheme 20

Figure 2.8 CVD process scheme 20

Figure 2.9. Photolithography process 21

Figure 2.10 Etching types process 22

Figure 2.11 MEMS fabrication Example 22

Figure 2.12 Piezoelectric MEMS example 23

Figure 2.13 Distribution of piezoresistive elements on the substrate 24

Figure 2.14 Capacitive beam resonators 24

Figure 2.15. a DEFT Resonator, b. DEFT Accelerometer. 25

Figure 2.16 Active piezoelectric tactile sensor 26

Figure 2.17 Strain gauges structure. 26

Figure 2.18 Packaging levels 27

Figure 2.19 Zero level package 28

Figure 2.21 Connections techniques 29

Figure 2.22 Ceramic package level 1 fabrication 29

Figure 2.23 Metal packages $\quad 30$

Figure 2.24 Plastic packages used in communications industry $\quad 30$

Figure 2.25 SAW MEMS Device. 33

Figure 3.1 SAW structure and its dimensions. 36

Figure 3.2 Substrate deformation shape per period. 36

Figure 3.3 Wireless SAW sensor mode. 36

Figure 3.4 SAW resonator configurations 37

Figure 3.5 Two-port delay line configuration. 38

Figure 3.6. Dimension characteristics of an SAW two port resonators. 38

Figure 3.7 Finger dimensions of an SAW IDT 40

Figure 3.8 Longitudinal and shear waves 41

Figure 3.9 Propagation zones of a wave 41

Figure 3.10 Left and right crystal quartz 46

Figure 3.11 Angle between faces of a crystal 46

Figure 3.13. Rotation operations a. Types, b. Restrictions 48

Figure 3.14 Three Fold rotation representation 48

Figure 3.15 Crystal systems 49

Figure 3.16 Crystal standards definitions. @Comsol. 50 
Figure 3.17. Quartz crystal characteristics 51

Figure 3.18 Wave power spreading 52

Figure 3.19. Surface acoustic wave 52

Figure 3.20. Particle displacement 53

Table 3.3 SAW properties and applications 53

Figure 3.21 Acoustic wave modes relations.

Figure 4.1 PVA film preparation in molds 58

Figure 4.2 The SAW resonator unit without packaging type TO-39 59

Figure 4.3 The SAW resonator before the PVA film and with the

PVA film. $\quad 59$

Figure 4.4. PVA film for mechanical test 60

Figure 4.5 PVA films mechanical test 60

Figure 4.6 Example of a Tension stress- strain curve for sample $1 \quad 61$

Figure 4.8.a Colpitts schematic circuit. b. Schematic circuit

and real oscillator circuit (top and bottom views). 63

Figure 4.9 Diagram of the measurement system for the SAW

humidity sensor $\quad 64$

Figure 4.10 Experimental setup for humidity measurements. 65

Figure 4.11 Test $1 \quad 67$

Figure 4.12 Test $2 \quad 68$

Figure 4.13 Test $3 \quad 69$

Figure 4.14. Test $4 \quad 70$

Figure 4.18 Frequency shift results using partial density method compared to Experimental SAW Humidity sensor $\quad 73$

Figure 5.1 Photo of SAW dimensions $\quad 75$

Figure 5.2 Model dimensions used in the simulations. 76

$\begin{array}{ll}\text { Figure 5.3 Mesh and boundary conditions applied } & 77\end{array}$

Figure 5.4 Symmetric and antisymmetric SAW modes 78

Figure 5.5 SAW admittance $\quad 79$

Figure 5.6 Numerical and experimental results comparisons $\quad 80$ 


\section{List of Tables}

Table 2.1 Mechanical and Electrical properties of MEMS materials. 15

Table 2.3 Humidity sensors review 32

Table 2.2 MEMS sensing principle general comparison. 24

Table 3.1 Crystal system and classes 49

Table 3.2 Piezoelectric substrate properties 51

Table 3.4 Different Wave types performance comparison 55

Table 4.1 Different acoustic wave sensors properties 56

Table 4.2 Young modulus of the 15 samples in GPA. 61

Table 4.3 Electrical specifications of the D02. 62

Table 4.4 Operation parameters of characterization tests 66

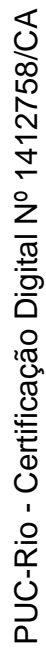

Table 5.2 PVA material properties $\quad 76$

Table 5.3 Boundary conditions of the simulation model 77 


\section{Introduction}

\section{1}

\section{Problem definition}

In many cases, humidity measurements are essential to control the environment atmosphere in chemical reactions and physical processes. High temperatures, corrosive substances or difficult access could make this objective hard to achieve with conventional sensors. However, the Micro ElectroMechanical Systems (MEMS) technology evolution have been providing a wide range of sensors that represent a possible solution for this purpose.

\section{2}

\section{Motivation and Objectives}

MEMS sensors technology have been in a progressive product development driven mostly by the smartphone or internet of things industry. Successfully implemented sensors vary from inertial motion sensors to pressure sensors, which typically are based on piezoelectric, piezoresistive and capacitive principles. Surface Acoustic Wave (SAW) devices are an example of MEMS grounded on the piezoelectric principle, which makes it capable of being passive structures, meaning that a continuous energy supply or batteries shouldn't be necessary. Instead, it can be energized through a wireless RF pulse and communicate in wireless mode. Another important feature is the harsh environment resistant properties, which provides ability to work in temperatures ranging from $-150^{\circ} \mathrm{C}$ to $300^{\circ} \mathrm{C}$.

In this study, humidity-monitoring sensor based on SAW MEMS technology was developed. Experimental results were compared with an analytical model and a finite element simulation in order to evaluate the sensor behavior and technology feasibility. 
Aiming to perform humidity measurements, the followings tasks were implemented:

- Conduct a bibliographic review about MEMS technology

- Research about SAW devices and applications

- Develop a humidity sensor based on SAW resonator

- Perform humidity sensor tests in a climatic chamber

- Study an analytical model of SAW gas sensors

- Evaluate the SAW frequency response through a numerical analysis as a function of relative humidity

- Comparison between experimental, analytical and numerical results.

\section{3}

\section{Dissertation outline}

This work is divided in 6 chapters, including the introduction as chapter 1 . In chapter 2 a bibliographic review is carried out about MEMS technology, with a brief consideration about fabrication processes, sensing principles and packaging technologies, also some commercial MEMS examples and applications are shown.

In chapter 3 the theory related to SAW devices is presented, their design and physics, specifically the piezoelectric effect and crystal structure are treated. Also acoustic wave modes and some applications are shown.

In chapter 4 the experimental set up used is described, showing the climatic chamber, circuit layout and signal processing. In addition, the different tests carried out are listed, giving their characteristic humidity and temperature variation. Finally, test results are presented together with a comparison of the sensor performance with an analytical model studied for the PVA coated SAW humidity sensor.

Finally, in chapter 5 the finite element simulation carried out in the Comsol Multiphysics software is presented. All the analysis, boundary conditions, loads applied, material specifications and mesh used are described. Simulation results and a comparison against the experimental test are also shown here. 


\section{2}

\section{MEMS Technology}

MEMS (Micro electro mechanical systems) are sensing or actuator devices made on a micrometric scale and mostly of a silicon substrate. But, for harsh environment applications other substrate materials are preferred [1] due to more adequate properties, as shown in table 2.1. MEMS devices combine mechanical (e.g. plates, beams) with electrical structures (circuit) [2], integrated through the use of microfabrication techniques adapted from the semiconductor industry. This allows the implementation of complete miniaturized systems such as the one depicted in figure 2.1.

Table 2.1 Mechanical and Electrical properties of MEMS materials. (Azevedo, 2011).

\begin{tabular}{|c|c|c|c|c|}
\hline Property & $\mathbf{S i}$ & $\mathbf{S i}_{3} \mathbf{N i}_{4}$ & Diamond & SiC $^{*}$ \\
\hline Young's Modulus E [GPa] & 190 & 304 & 1035 & 448 \\
\hline Density, [Kg/cm ${ }^{3}$ ] & 2330 & 3300 & 3510 & 3300 \\
\hline Fracture strength, [GPa] & $2-4$ & $5-8$ & $8-10$ & $4-10$ \\
\hline E/p, [GN/Kg.m] & 72 & 92 & 295 & 130 \\
\hline
\end{tabular}

\begin{tabular}{|c|c|c|c|}
\hline Property & $\begin{array}{c}\text { Energy } \\
\text { Bandgap [cV] }\end{array}$ & $\begin{array}{c}\text { Electron } \\
\text { Mobility }\end{array}$ & $\begin{array}{c}\text { Relative Dielectric } \\
\text { constant }\end{array}$ \\
\hline Si & 1,12 & 1200 & 11,9 \\
\hline GaAs $^{*}$ & 1,43 & 6500 & 13,1 \\
\hline
\end{tabular}

* Materials: Gallium arsenide "GaAs", Silicon carbide "SiC".

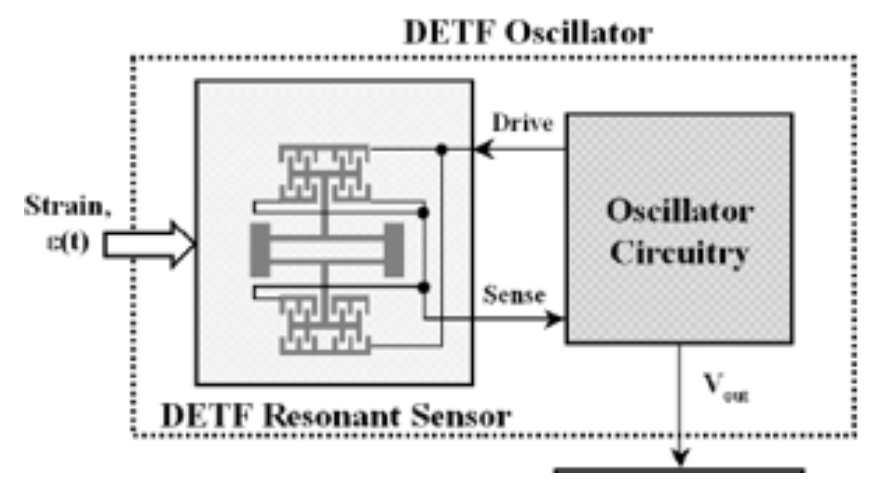


Figure 2.1 Schematic structure of MEMS (Ahmed, 2006).

The history of MEMS is linked to the Integrated Circuit (IC) industry. In fact, it could be considered as its spin-off, since many of the fabrication processes currently used are derived from the semiconductor development in the fifties. In figure 2.2 is shown a timeline for the MEMS technology development.

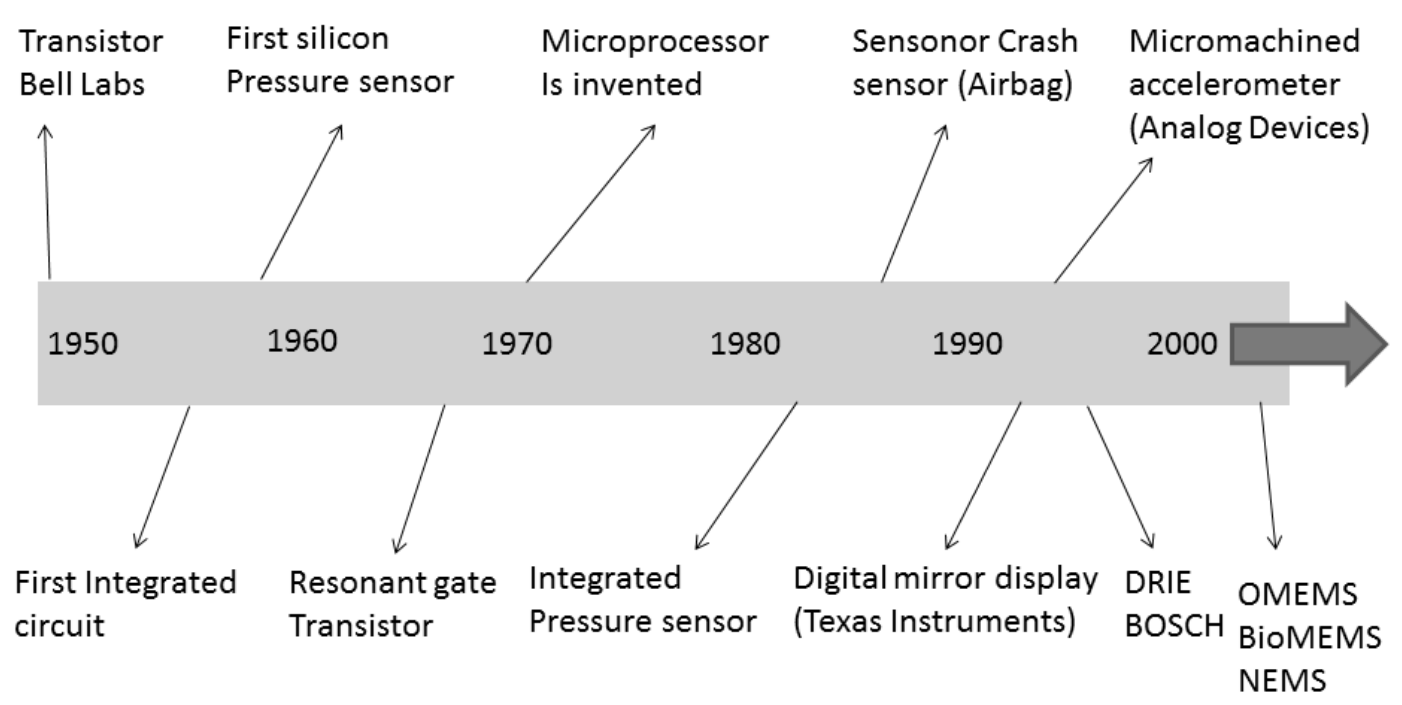

Figure 2.2 History of MEMS

Some of the milestones of MEMS development are pointed out below:

In 1948, the invention of the Germanium transistor at Bell Labs (William Shockley) started a revolution on the electronic world as everything could be condensed then in small devices, which would be the philosophy of the micromachining process leading the inventions of the MEMS later.

Furthermore in 1954, the Piezoresistive effect in Germanium and Silicon was found by C.S. Smith, and in 1958 the first integrated circuit (IC) was built by J.S. Kilby and Robert Noyce. In 1959, the famous talk given by R. Feynman, "There's Plenty of Room at the Bottom" about the micromachines, changed the way the world imagined them, showing that it was not a science fiction movie anymore.

The first silicon pressure sensor demonstrated in 1959 by Kulite and the Resonant Gate Transistor Patented in 1968 by H. Nathanson et.al, confirmed Feynman's forecast. In the following years, the use of Surface and Bulk Micromachining Processes created in the 70's, allowed pressure sensors to be fabricated in Bulk etched silicon wafers, grounding the next micro-devices generation. Other important facts are shown below:

- In 1971: invention of the microprocessor; 
- In 1979: HP created a micromachined ink-jet nozzle used in printing machines;

- In 1982: Disposable blood pressure transducer;

- In 1983: Integrated pressure sensor by Honeywell;

- In 1985: the first Crash sensor (Airbag) by Sensonor;

- In 1988: Batch fabricated pressure sensors via wafer bonding by Nova Sensor;

- In 1993: Digital mirror display by Texas Instruments;

- In 1993: First surface micromachined accelerometer in high volume production by Analog Devices; and

- From the 2000's the optical MEMS and BioMEMS boom.

Later on some process like the LIGA process (acronym for $\mathrm{x}$-ray electroplating and molding in German) by KFK in 1982 Germany, the Silicon wafer bonding by M. Shimbo in 1986, and the Bosch process for Deep Reactive Ion Etching patented in 1994, were created. In addition, the MUMP (Multi-user MEMS process) created in a foundry service by MCNC (Microelectronics Center in North Caroline) in 1993 intended to standardize MEMS fabrication. All of them were fundamental parts of the MEMS history.

\section{1}

\section{MEMS fabrication process}

Many of the MEMS fabrication processes have been adapted from the semiconductor industry [3]. Their fabrication begin with the addition of one by one subsequent structural or insulator layers over a silicon substrate, until the final MEMS distribution is completed. Later on patterning process implants the design model in the superficial layer and finally the etching process transfers this pattern to the subsequent layers, as can be seen in figure 2.3.

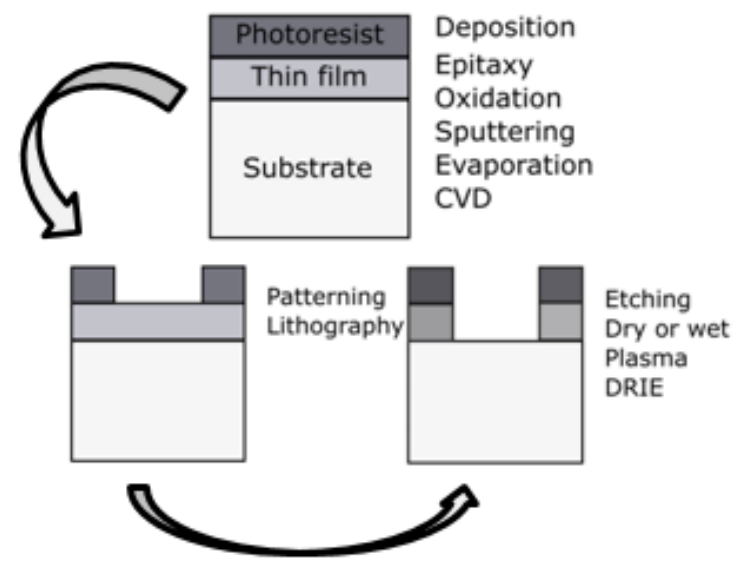


Figure 2.3 Typical MEMS fabrication process

In the following sections a brief review of these processes are presented.

\subsection{1}

\section{Deposition methods:}

The first stage in MEMS fabrication is the insertion of layers through deposition methods, some of these processes are going to be described together with their schematic representation, as follows.

\subsubsection{1}

\section{Epitaxy:}

Epitaxy is referred to the deposition of atoms in a crystal form upon a crystal substrate. Process is carried out in a vapor phase chemical deposition reactor, as shown in figure 2.4. A dissociation or hydrogen reduction occurs at high temperatures, while silicon tetrachloride $\mathrm{SiCl}_{4}$ is one of the types of source gas commonly employed to form the epitaxial layers [1].

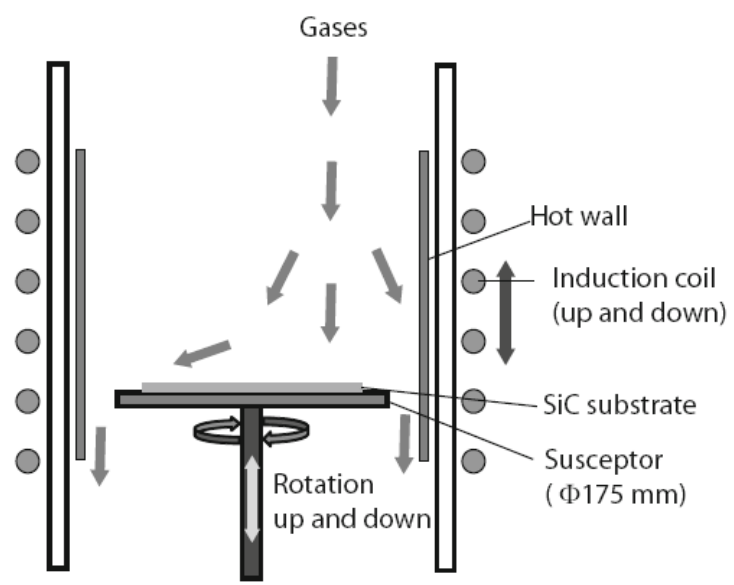

Figure 2.4 Epitaxy process scheme (Wijesundara, 2011).

\subsubsection{2}

\section{Oxidation:}

In this process a silicon dioxide layer is deposited over the substrate surface [4], this material is a high-quality electrical insulator used as a barrier material. The reaction to oxidize the substrate wafer is achieved heating it in an atmosphere of 
pure oxygen or water vapor, at temperatures of $700-1,200^{\circ} \mathrm{C}$, as shown in figure 2.5 .

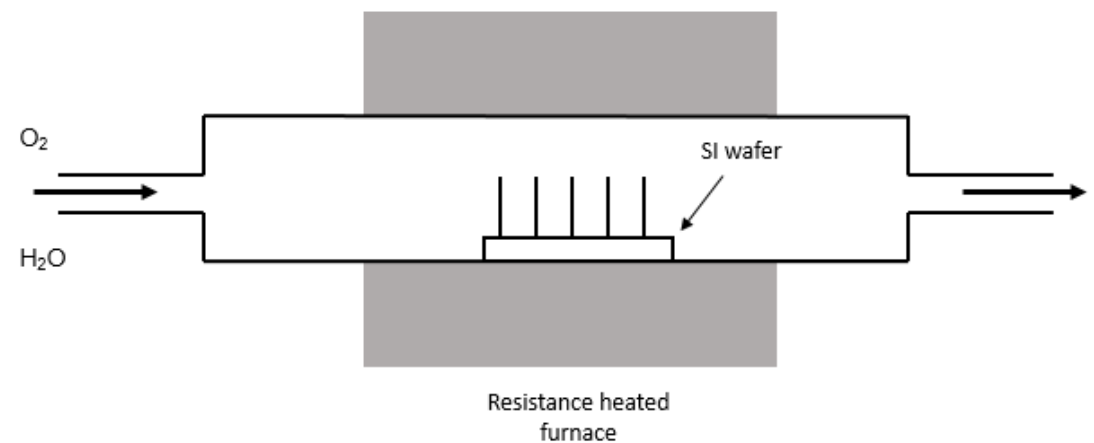

Figure 2.5 Oxidation chamber (Hu, 2009)

\subsubsection{3}

\section{Sputtering:}

The target material to be deposited is physically bombarded by a flux of inert gas ions (usually argon) [4] as shown in figure 2.6. Atoms are ejected towards the silicon wafer and deposited on it in a vacuum chamber. Usually Direct Current (DC) power supply can be used when depositing metals, but an Radio Frequency (RF) pulse supply is necessary when depositing insulating films.

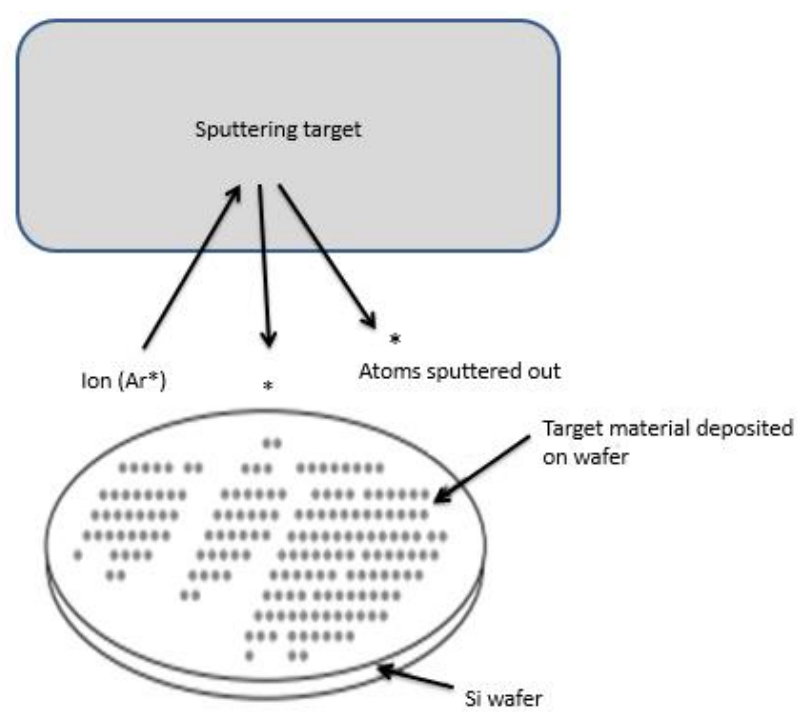

Figure 2.6 Sputtering process (Hu, 2009) 


\subsubsection{4}

\section{Evaporation:}

This process consists of heating a source material to generate the vapor that is later deposited on a substrate, forming the film of the target material in a vacuum chamber, as shown in Figure 2.7.

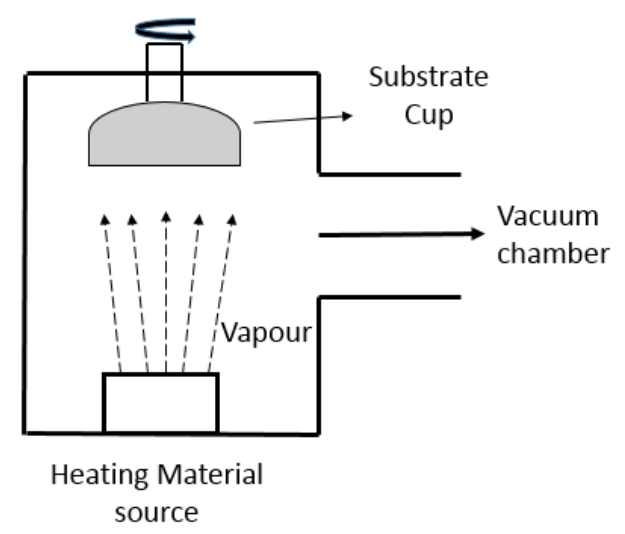

Figure 2.7 Evaporation process scheme (Jeol, 2015).

\subsubsection{5 \\ Chemical Vapor Deposition (CVD):}

A chemical reaction between two source gases is undertaken in a controlled atmosphere over the surface of a heated substrate (above $3000^{\circ} \mathrm{C}$ ), as shown in figure 2.8. This process is commonly at atmospheric pressure (APCVD), Low pressure (LPCVD) or with Plasma Enhanced (PECVD). CVD process is routinely used to deposit films of $\mathrm{SiO}_{2}, \mathrm{Si}_{3} \mathrm{~N}_{4}$, and dielectrics with excellent chemical and electrical stability [4].

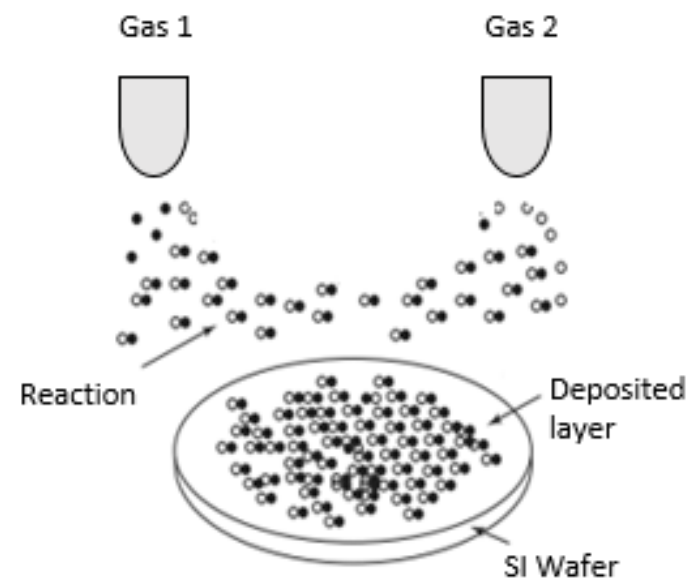

Figure 2.8 CVD process scheme (Hu, 2009). 


\subsection{2}

\section{Patterning:}

The way through which a designed distribution of structures is implanted in the superficial layers of a MEMS, is typically by the use of patterning processes. A brief description of the most common type of pattering is given below.

\subsubsection{1 \\ Photolithography:}

The removal of selective areas is done through the application of UV light over a photoresist material and through the chemical reaction with a solvent. The removal of the exposed or unexposed regions depends on the type of resist used (positive or negative), as can be seen in figure 1.9. The pattern to be formed is transmitted by a photomask with opaque regions blocking the UV light, leading to protect the region and thus no reaction is produced [4].

a.

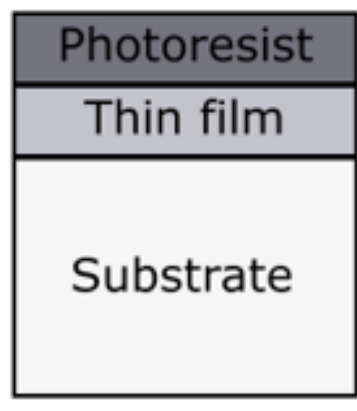

b.

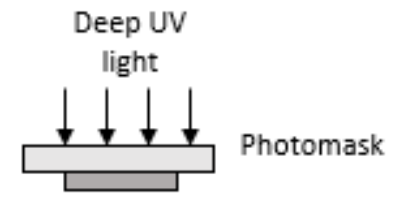

Positive resist

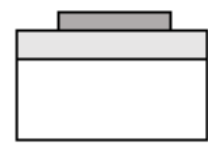

Negative resist

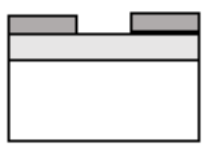

Figure 2.9. Photolithography process: (a) application of resistive material; (b) exposure through a mask and development of exposed photoresist (Hu, 2009).

\subsection{3}

\section{Etching:}

In order to form a functional MEMS structure on a substrate, it is necessary to etch the thin films previously deposited and patterned. In general, there are two classes of etching processes, dry and wet etching as described below. 


\subsubsection{1 \\ Dry Or Wet etching:}

This pattern formed by lithography is often transferred to underlying layers in two ways, the first is called wet etching if this material is removed with acid (isotropic if there is not preferential direction) [5]. The second is dry etching - called plasma etching or Reactive lon Etching (RIE) as well - these ions react chemically with the material to be etched, in this case, material removal is preferentially vertical and the etch rate is anisotropic, as shown in figure 2.10 .

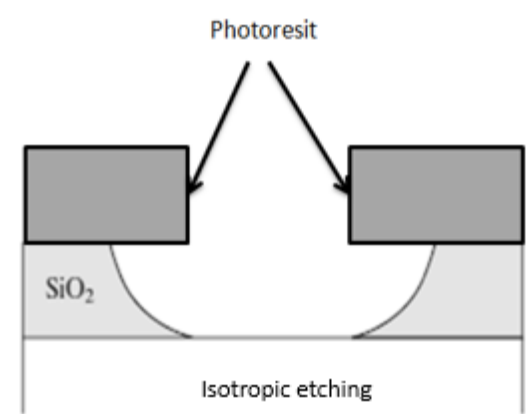

a.

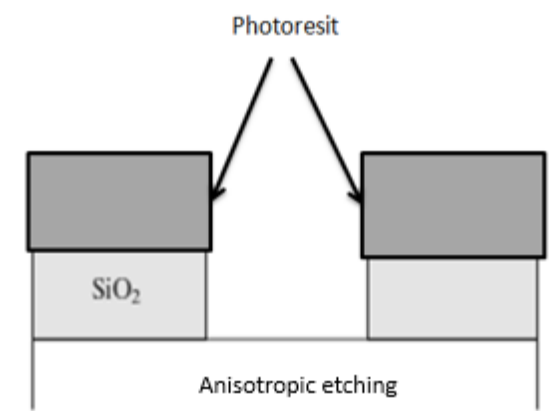

b.

Figure 2.10 Etching types of etching process: a. Normally the acid used in wet etching is Fluoridric acid (HF), b. in dry etching are fluorine or chlorine-containing plasmas.

One example of a MEMS fabrication process is shown in figure 2.11 with the steps to produce it.

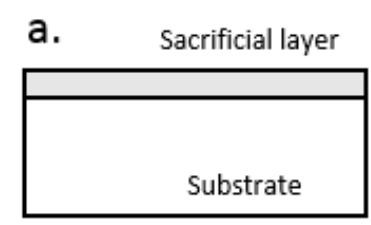

b.

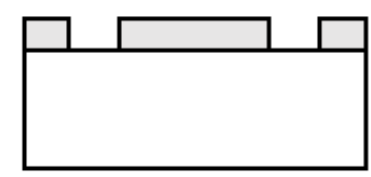

C. Structural layer

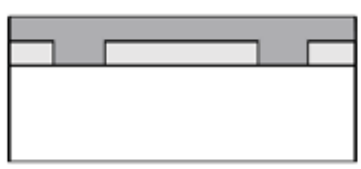

d.

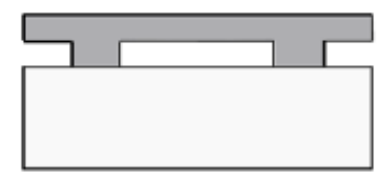

Figure 2.11 MEMS fabrication Example. a. Deposition, b. Patterning, c. Sputtering, d. Etching. 


\section{2}

\section{MEMS sensing principle}

MEMS sensors are mainly based on piezoelectric, piezoresistive and capacitive technologies. The first two (piezoresistive and piezoelectric effects) use material properties to detect changes in the variable(s) of interest, these three principles are explained below.

\subsection{1}

\section{Piezoelectric MEMS:}

This sensing method uses electrical material characteristics to detect changes in the variable to be measured. The material generates an electrical field when the substrate is subjected to deformation or mechanical force, as shown in figure 1.12. It is interesting to many applications in which passive elements are needed or energy supply is limited. This effect can be reversible, since the material expands or contracts in response to an externally applied voltage [6].

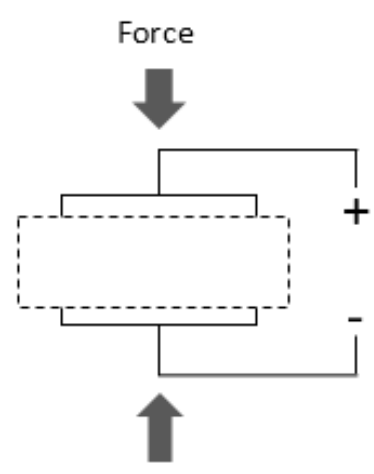

Figure 2.12 Piezoelectric MEMS example

\subsection{2}

Piezoresistive MEMS:

In this case, application of any mechanical force or deformation varies the resistance of the material [7]. An external circuit senses the variation of this doped materials, as shown in figure 2.13. The disadvantage is the need of a constant voltage supply to keep it working in most applications. 


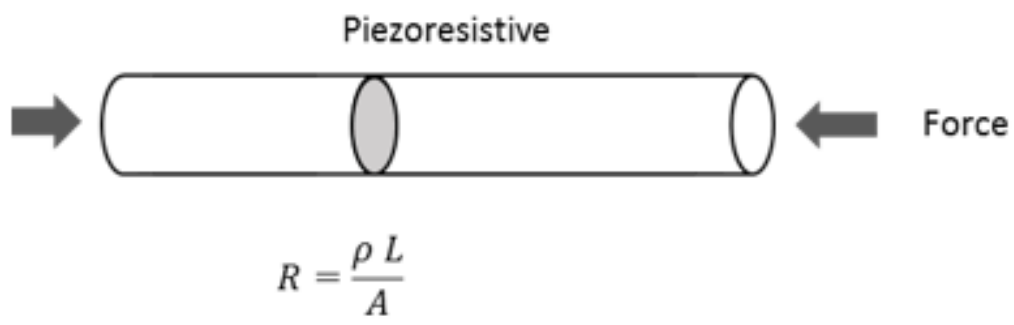

Figure 2.13 Distribution of piezoresistive elements on the substrate

\subsection{3}

\section{Capacitive MEMS:}

These devices use the variation of the capacitance in the gap between two plates [8], when a DC voltage is applied to the structure, as shown in figure 2.14. An external circuit senses this capacitance shift and the variable can be obtained through correlations.

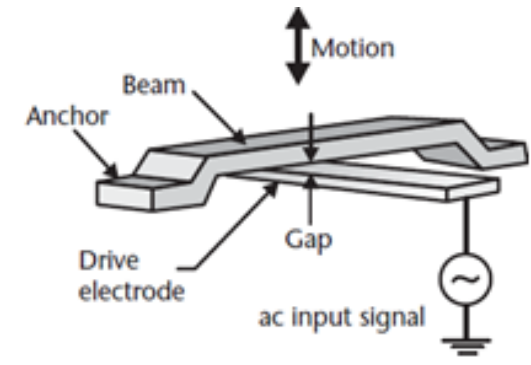

Figure 2.14 Capacitive beam resonators (Debbie G. Jones, et.al. 2011).

The table 2.2 presents a comparison of general sensing parameters of piezoresistive, piezoelectric and capacitive sensor[9][10]

Table 2.2 MEMS sensing principle general comparison.

\begin{tabular}{|c|c|c|c|}
\hline Measuring range & Piezoresitive & Piezoelectric & Capacitive \\
\hline Sensitivity & High & Wide & Limited \\
\hline $\begin{array}{c}\text { Temperature } \\
\text { Dependance }\end{array}$ & High & High & Low \\
\hline $\begin{array}{c}\text { Conditioning } \\
\text { circuitry }\end{array}$ & Simple & Complex & Complex \\
\hline $\begin{array}{c}\text { Consumed } \\
\text { power }\end{array}$ & Low & Low & High \\
\hline $\begin{array}{c}\text { Output signal } \\
\text { strength }\end{array}$ & High & High & Low \\
\hline
\end{tabular}




\begin{tabular}{|c|c|c|c|}
\hline Signal stability & Stable & Unstable & Stable \\
\hline Microfabrication & Well developed & Undeveloped & Developed \\
\hline
\end{tabular}

\subsection{4}

\section{Examples of MEMS sensors:}

Among the different sensing principles, one type of resonating structure is the Double Ended Tuning Fork (DETF). MEMS Devices with this structure bases their operation principle in the resonant frequency variation, induced by modifications in their structure dimensions (due to deformations or displacements) [1], to obtain the variable to be measured, as shown in figure 2.15.a. They are used in accelerometers [11] (figure 2.15.b), gyroscopes, pressure sensor, strain sensor and in RF communications as oscillators.

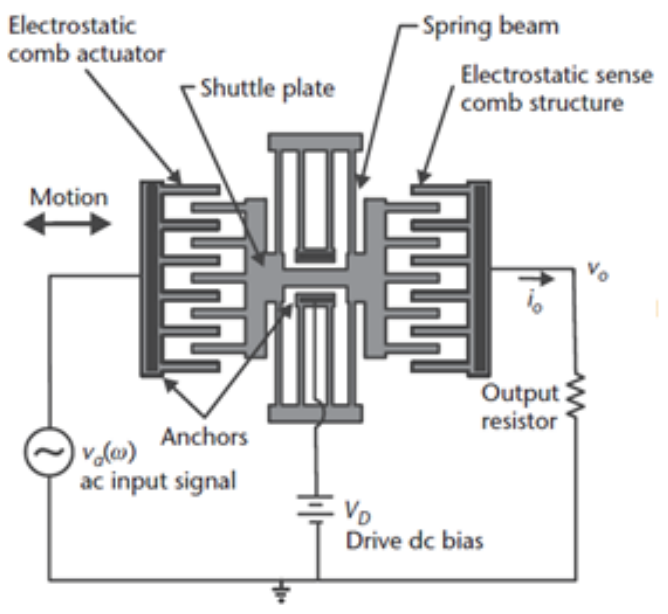

a.

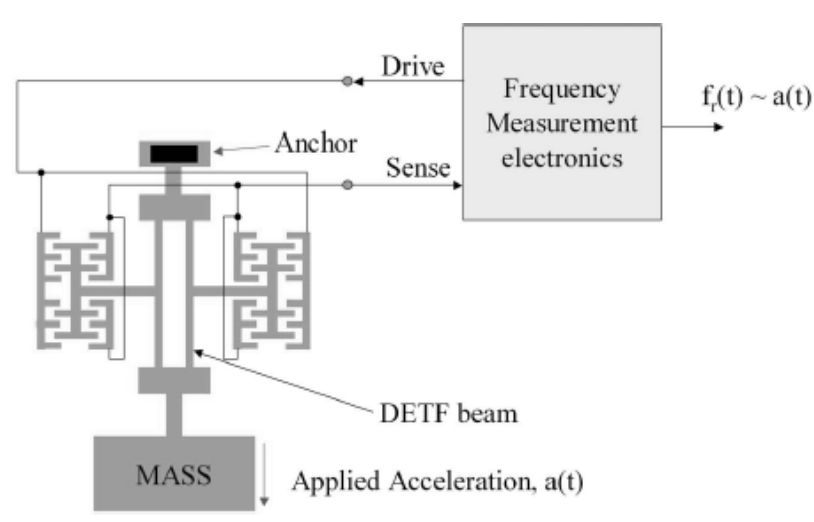

b.

Figure 2.15 a. DEFT Resonator, b. DEFT Accelerometer.

Active piezoelectric tactile sensor: This sensor can be fabricated with piezoelectric films and coupled acoustically with a center layer in a hamburger 
configuration, as shown in the figure 2.16. The alternate current (AC) signal produced by the oscillator generates a contraction between the top and bottom piezoelectric layers. When a force is applied to the upper layer, the mechanical coupling of the three layers is modified, affecting the amplitude and the phase of the output signal that can be detected as a variable voltage [12].

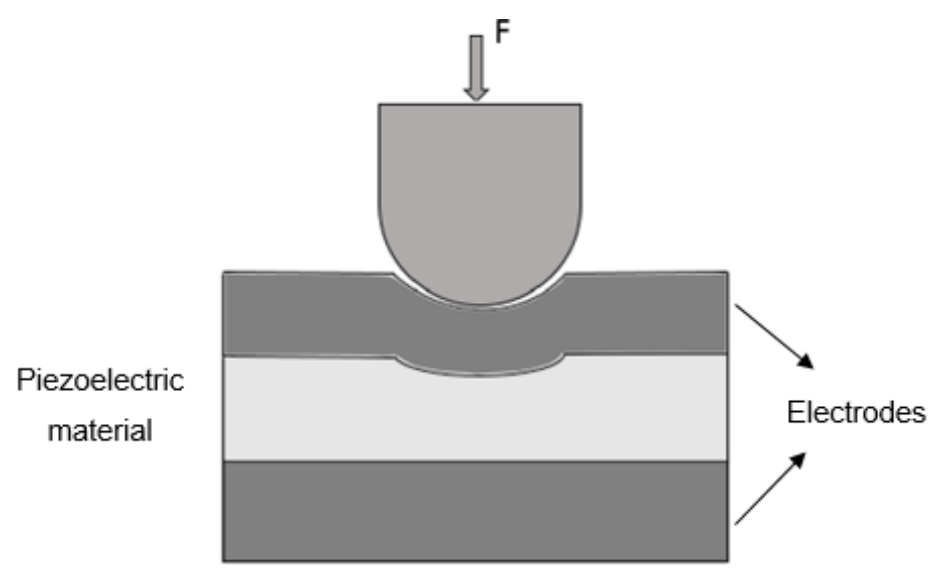

Figure 2.16 Active piezoelectric tactile sensor

Piezoresistive Strain gage: One of the first applications of piezoresistive materials, were as metal strain gauges to measure strain in structures [3]. In these devices the deformation of the substrate also induces deformation on the doped piezoresistive elements (shown in figure 2.17) laying on their surfaces. The voltage variation can be detected by an external circuit and correlated with the strain suffered by the structure.

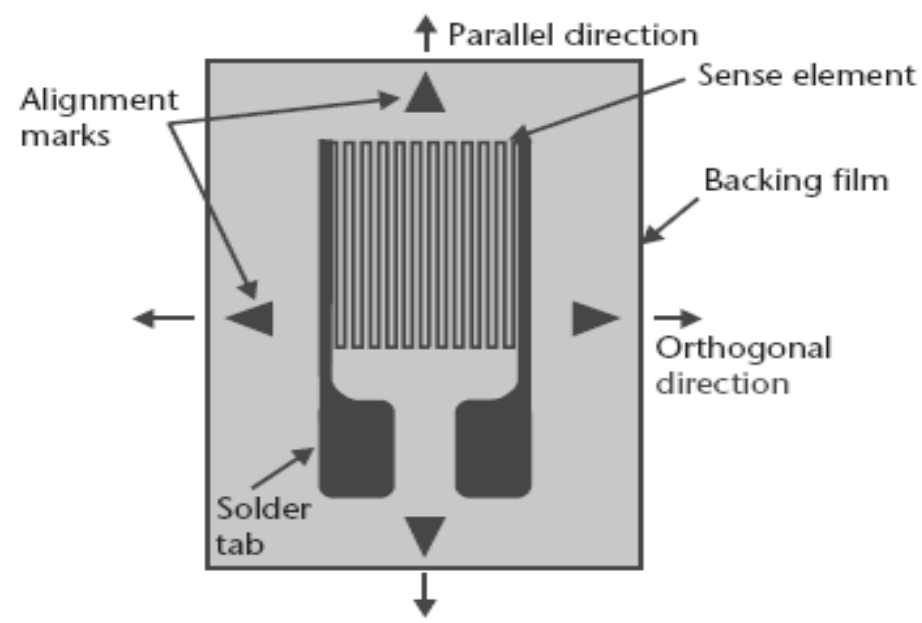

Figure 2.17 Strain gauges structure. 


\section{3}

\section{MEMS Packaging}

As an industry derived from semiconductor technology, MEMS packaging also has foundation in those packages implemented by the Integrated Circuit (IC) industry in all the electronics applications. This package is rarely a simple box casing the entire sensor; it is, for example: hermetic, vacuum-sealed and corrosion resistant structure. All these characteristics, in some cases, increase the price of the MEMS commercial products from 75 to $95 \%$ [1].

General packaging techniques vary according with the level of protection necessary, as shown in figure 2.18. They have protection at level 0 that covers the MEMS, passing to the level 1 that houses the MEMS, until the level 2 and 3 that are in the scale of the wafers package. Each type of level is described below.

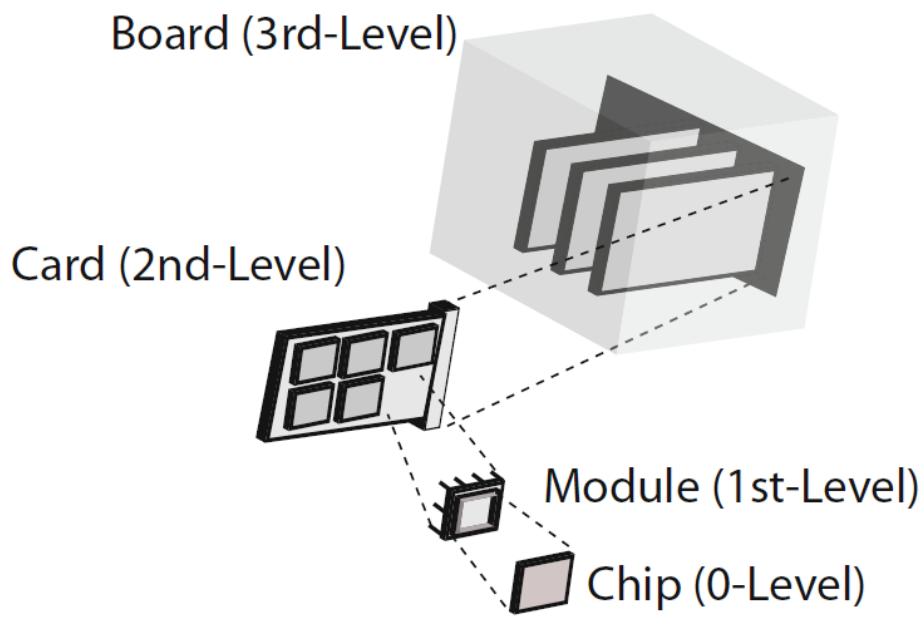

Figure 2.18 Packaging levels (Azevedo, 2011)

\subsection{1}

\section{Zero level package}

Techniques commonly used in this level are divided in two classes: bonding techniques and deposition techniques. In the former technique, a lid with a cavity is placed and bonded over the MEMS device, as shown in figure 2.19.a. In the last technique, additional layers are added over the top layer of the MEMS to place a lid or to form a corrosion resistant coating, as depicted in figure 2.19.b. 


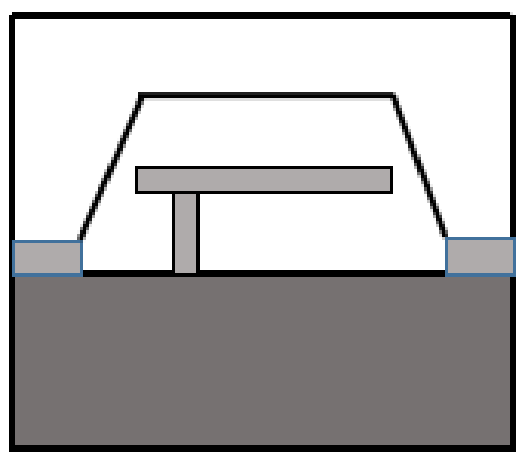

a.

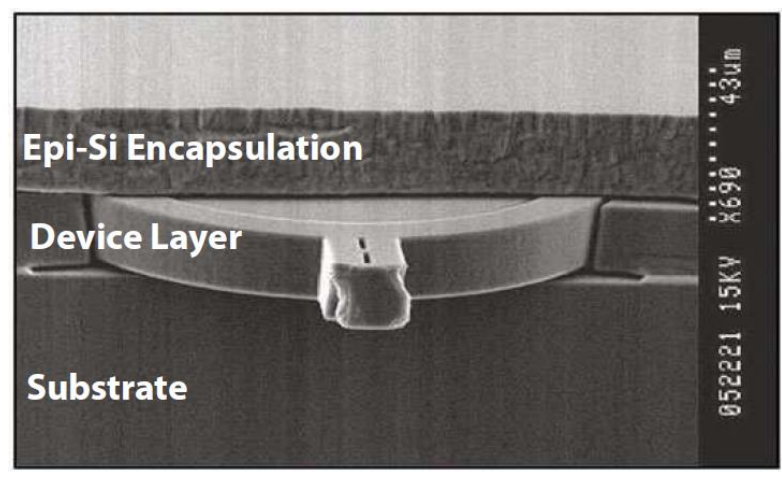

b.

Figure 2.19. Zero level package [1] a. Vacuum zero level package, b. Casing layers.

\subsection{2}

\section{First Level packaging:}

This level complements 0 -Level package as a module interconnected to a printed circuit board, that is inserted into a socket or by direct soldering, as shown in figure 2.20 .

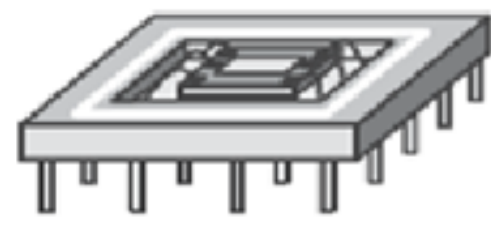

Wire bond

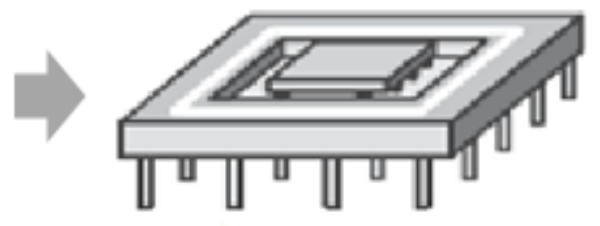

Flip chip

Figure 2.20 Flip-chip package [3].

The electrical connection is made with bump-bonding, as displayed in figure 2.21.a or wire bonding (usually gold material) [3], as shown in figure 2.21.b. 


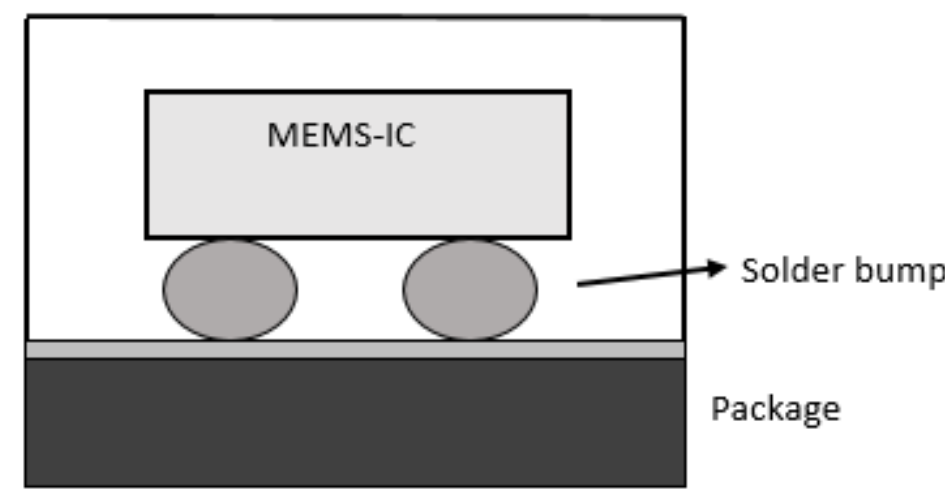

a.

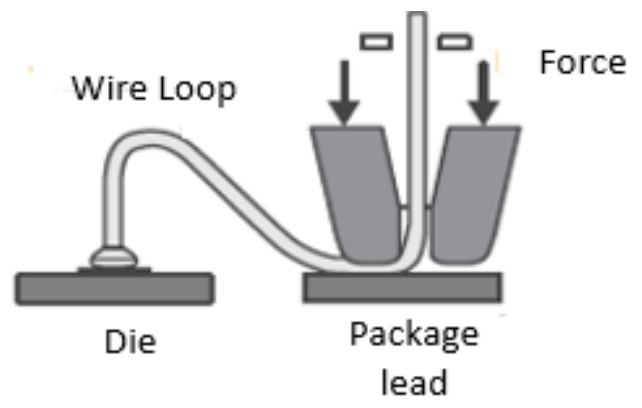

b.

Figure 2.21. Connections techniques: a. Bump bonding b. Wire bonding (Nadim, 2004)

\subsection{3}

Packaging types

Three categories can be distinguished regarding the material used for these MEMS packages.

Ceramic package: As shown in Figure 2.22, they are more expensive than a plastic package but less than a metallic one [3]. They are completely customizable and commonly used material is Alumina $\left(\mathrm{AL}_{2} \mathrm{O}_{3}\right)$.

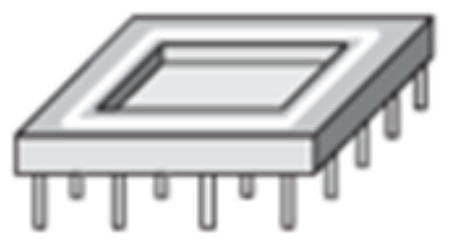

Figure 2.22 Ceramic package level 1 fabrication (Nadim, 2004).

Metal package: They are hermetic when sealed and can cost almost 10 times a plastic one, usually over US\$1 [3]. The material frequently used is stainless steel as shown in figure 2.23, especially in applications with contact with the environment, for instance, pressure measurements in flow lines of industrial fluids. 

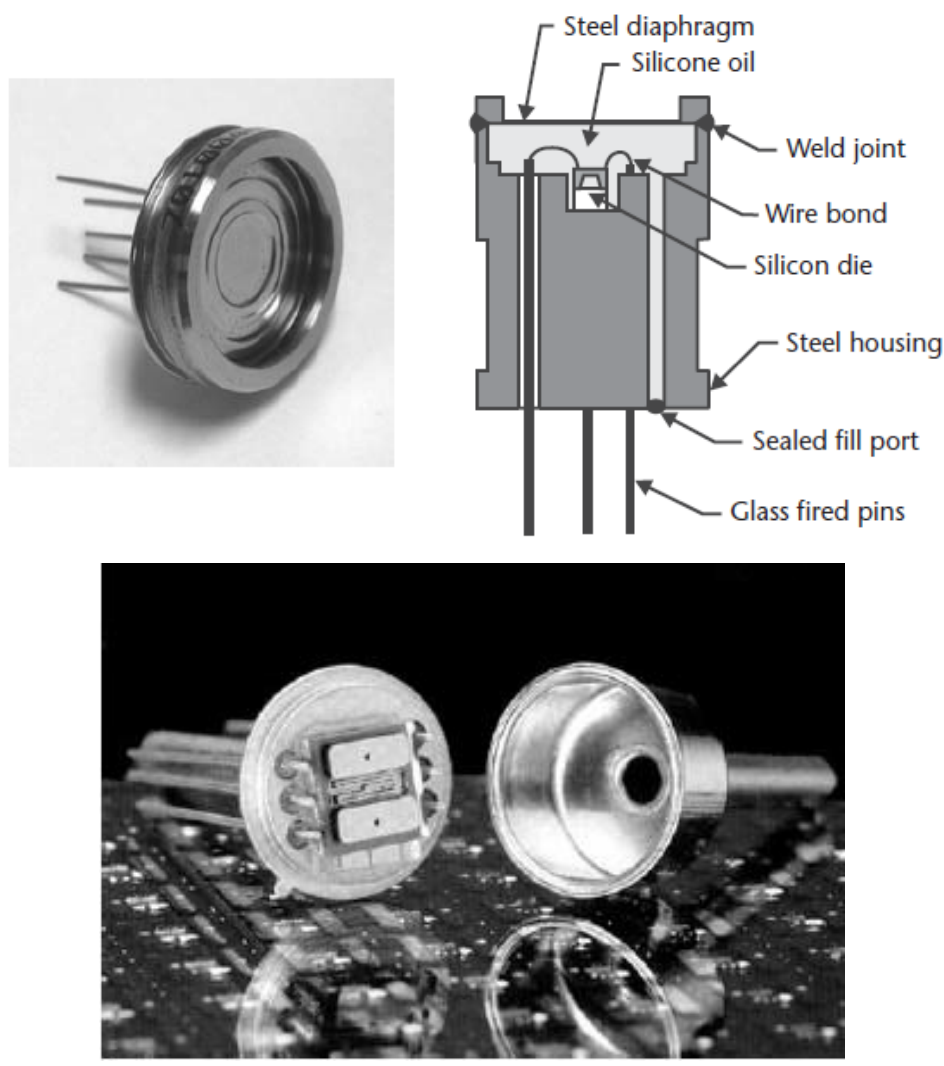

Figure 2.23 Metal packages (Nadim, 2004).

Plastic package: This is a low cost and sometimes small size solution package, but unfortunately also inadequate for harsh environments. Rather than the others, this package type is not an hermetic one. For instance, commercial pressure or acceleration sensors package is usually below US $\$ 5$, and the material frequently used is an epoxy such as Novolac, which is preferred due to its improved heat resistance. Some of these packages are shown in figure 2.24.

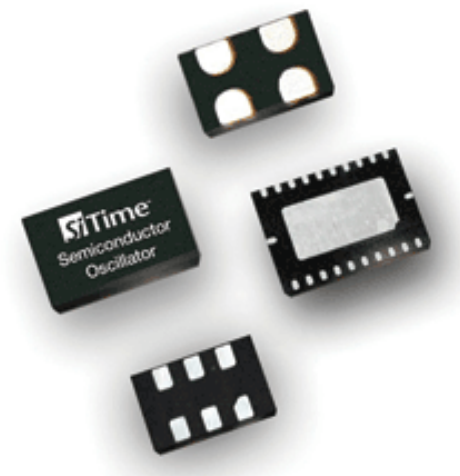

Figure 2.24 Plastic packages used in communications industry, @SITime 
Then, depending on the MEMS application, the decision about which package to choose varies between the work conditions, cost, life cycle and even the MEMS design itself.

\section{4}

\section{Application to Humidity measurements}

Humidity is defined as the amount of water vapour in an atmosphere of air or other gases and it is used in several industry applications such as chemical processing, environmental monitoring, agriculture, medical and laboratory instrumentation, semiconductors and even the Internet of Things (IoT). Humidity sensors have gained attention as a tool to increase devices and processes performance.

The different sensors commercially available fulfill particular operation conditions from each field, depending also in which type of measurement technique are used, if it is a relative or an absolute humidity measure, having the following common units:

Relative humidity $(\mathrm{RH})$ : This measurement expressed as a percentage, represents the ratio of the partial pressure of water vapour in a solution $P_{v}$, to the water vapour saturation pressure at a given temperature $P_{s}$. Because $\mathrm{RH}$ is dependent of temperature then it is a relative measurement. This type of measurement covers applications with higher humidity ranges and is given by:

$$
\% \mathrm{RH}=\frac{P_{v}}{P_{S}} * 100
$$

Dew/Frost point (D/F PT): These are temperature points, at which the water vapour condenses to liquid water referring to Dew point (above $0^{\circ} \mathrm{C}$ ) and when water vapour condenses to ice is referred to the Frost point (below $0^{\circ} \mathrm{C}$ ). The difference with the above $\mathrm{RH}$ measure is that, this measure is a function of the gas pressure and is independent of temperature, thus it can be called as absolute humidity measurement. This type can cover applications in the entire humidity range.

Parts Per Million (PPM): This is another absolute measurement representing the water vapour content by volume fraction or by the ratio of water molecular weight to that of air. This type of measurement covers applications with low humidity range. 
Absolute humidity in general is defined as a ratio of water vapour mass in air to the volume of air:

$$
A B=\frac{m_{v}}{v}
$$

Thus, sensors are then separated into relative and absolute humidity sensors with different sensing materials and detection ranges, where the first type $\mathrm{RH}$ are based on ceramic, semiconductor and polymer materials. The second type also named hygrometers are commonly solid moisture and mirror chilled hygrometers, which are compared in the following table 2.3.

Table 2.3 Humidity sensors review [13][14]

\begin{tabular}{|c|c|c|c|c|c|c|c|}
\hline \multirow{2}{*}{ Type } & \multirow{2}{*}{$\begin{array}{l}\text { Fabrication } \\
\text { technology }\end{array}$} & \multicolumn{3}{|c|}{$\begin{array}{l}\text { Sensing } \\
\text { Material }\end{array}$} & \multicolumn{2}{|c|}{$\begin{array}{c}\text { Transduction } \\
\text { type }\end{array}$} & \multirow[t]{2}{*}{ Cost } \\
\hline & & El & Pol & Cer & $\mathbf{R}$ & C & \\
\hline \multirow{4}{*}{$\mathbf{R H}$} & $\begin{array}{c}\text { Conventional } \\
\text { Ceramic/Semicondutor } \\
\text { Processing }\end{array}$ & $A$ & NA & A & A & $A$ & Low \\
\hline & Thick Film, LTCC & NA & $A$ & A & A & NA & Medium \\
\hline & Thin Film & NA & $A$ & A & A & $A$ & Medium \\
\hline & p-n Heterojunction & NA & NA & A & A & $A$ & $\begin{array}{c}\text { Medium } \\
\text { High }\end{array}$ \\
\hline \multirow{2}{*}{ AB } & Solid Moisture $\left(\mathrm{Al}_{2} \mathrm{O}_{3}\right)$ & NA & NA & A & & & $\begin{array}{c}\text { Medium } \\
\text { Low }\end{array}$ \\
\hline & $\begin{array}{c}\text { mirror chilled } \\
\text { D/F Point }\end{array}$ & NA & NA & NA & & & High \\
\hline
\end{tabular}

In the table NA means Not Available, A is Available, El is electrolyte, Pol is Polymers and Cer is Ceramics. Also $R$ and $C$ are the resistive and capacitive transduction mechanisms.

Most of sensors works with principles like proton conductive, characterized by changes in electrical conductivity on the surface (where electrons are 
concentrated), derived from chemical or physical absorption of water vapour molecules.

Other principle is the electronic or ionic type (charge carriers), classified into the conduction type sensors due to their electrical transport mechanism. In this case water adsorption changes the electrical properties like resistance, capacitance or electrolytic conduction [13], [14].

No matter which transduction type shown above is going to be used in the sensor, in all of them a sensing film is deposited over the structure having properties such as resistivity, dielectric constants or mass affected with humidity variations.

Finally, another transduction mechanism studied is the piezoelectric effect which is the principle behind the Acoustic Waves devices used for humidity or gas sensing [15]. Between these devices are the Surface Acoustic Wave (SAW) shown in figure 2.25. They use interdigitated electrodes that can excite, detect and reflect Rayleigh waves launched by an external RF pulse in a piezoelectric substrate.

If a sensing film is deposited over the substrate, then a wave velocity variation is detected when water vapour absorption occurs and the humidity can be measured through correlations. As the substrate is piezoelectric, the RF pulse finally is reconverted into electrical signal that can be transmitted wirelessly to a interrogation unit for analysis.

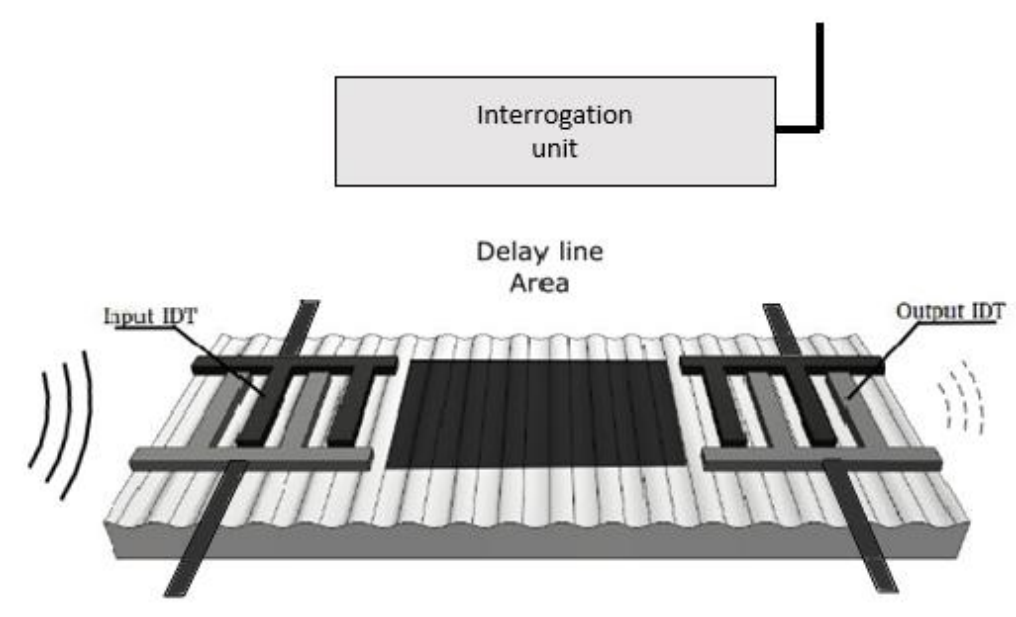

Figure 2.25 SAW MEMS Device.

Therefore, the Surface Acoustic Wave (SAW) devices have been chosen as the most suitable MEMS in this application having the following characteristics:

They are passive structures, which means do not need a continuous energy supply or batteries. In other words as they work with the piezoelectric principle, 
they can be energized and interrogated through a wireless Radio Frequency (RF) pulse in a wireless mode.

Another important feature is the small size with thin footprint and even environmentally tough, as they can work in temperatures varying from $-150{ }^{\circ} \mathrm{C}$ to $300{ }^{\circ} \mathrm{C}$ with no corrosion problems. Taking into account, these characteristics together with a low cost (if a mass production exist) was definitive to choose the SAWs to develop the humidity system, where detailed information about this technology is presented below. 


\section{3 \\ SAW Technology}

SAW devices consist of a system that generates a wave propagating along the plane surface of an elastic solid. This type of wave was discovered by Lord Rayleigh in 1885 [16]. These devices have gained importance in sensing applications as they have several excellent characteristics when compared to other technologies. Some of them are listed as follows: they can be passive structures that do not need constant power supply, can be interrogated wirelessly through an antenna enabling remote monitoring in extreme conditions (which is very useful for industries with harsh environments). Their design and fabrication in a scale of microns, makes it a small, compact and low cost component. They have outstanding stability and high sensitivity, compatibility with Complementary MetalOxide-Semiconductor (CMOS) integrated circuits technology, and finally also offers a real time response. [17]-[19]

For instance, a one port SAW resonator is shown in the figure 3.1, depicting that SAW devices consist of an input interdigital transducer (IDT), acting as a wave transmitter. In this case, this IDT also acts as an output IDT, being the receiver of the reflected waves. IDTs are like comb-finger electrode structure made of a metallic conductor material, deposited on a piezoelectric substrate. In addition, two arrays of reflectors consisting of periodic narrow metal-shorted electrodes electrically connected, are placed on both sides of the IDTs. In 1965, White and Voltmer [20] demonstrated the basic SAW IDT structure, and since then they have been used extensively for electronic analog signal processing working as filters.

In figure 3.1, $\mathrm{N}_{1}$ and $\mathrm{N}_{2}$ are the number of electrodes in the IDT and reflectors arrays, respectively. $P_{1}, w_{1}$ and $P_{2}, w_{2}$ are the pitch and width of the IDT and reflectors arrays,respectively. Finally, $s$ is the separation between arrays, and $A$ and $\mathrm{M}$ are the electrodes length 


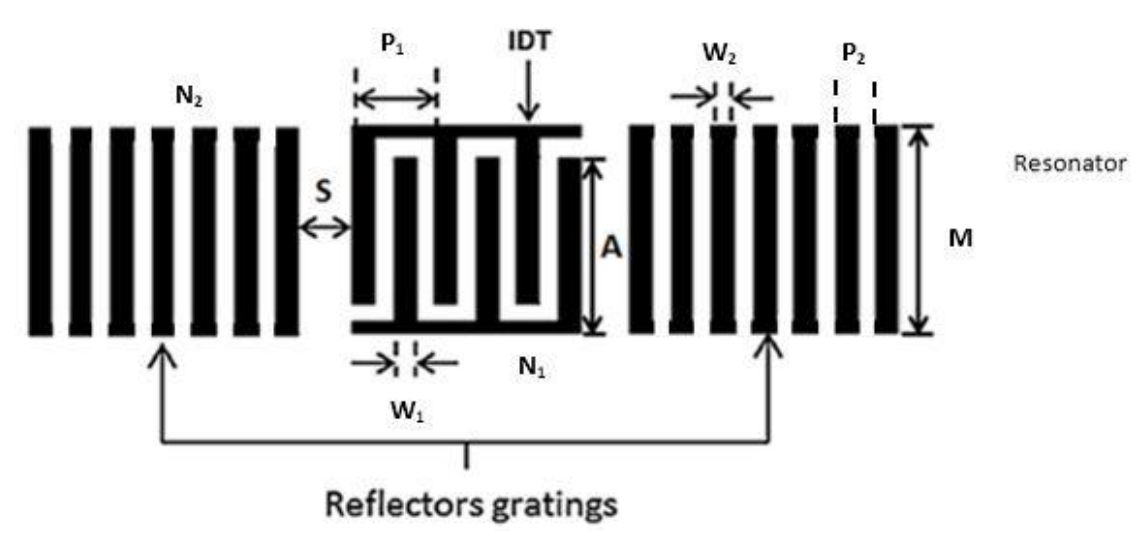

Figure 3.1 SAW structure and its dimensions.

The SAW works upon application of a voltage at the input IDT. Electrical charges accumulate at the IDT depending on the capacitance of the structure. The resulting electric field produced between the differently polarized fingers generates stress into the substrate due to the (reverse) piezoelectric effect. If an AC input voltage is applied, the continuously changing polarity of the charges will excite an SAW (Rayleigh wave) traveling through the substrate, as shown in figure 3.2.

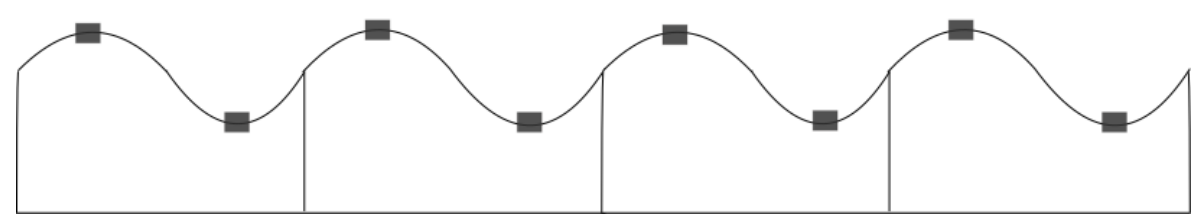

Figure 3.2 Substrate deformation shape per period.

This propagating mechanical wave is partly reflected back and partly transmitted by the array of reflectors on the sides and finally, the reflected train of SAW pulses is detected by the output IDT. Due to the current induced at the fingers by the piezoelectric effect, the wave is reconverted into sinusoidal electrical signal and transmitted through the antenna to the reader if wireless mode is used, as can be seen in figure 3.3 [21][22][23][24].

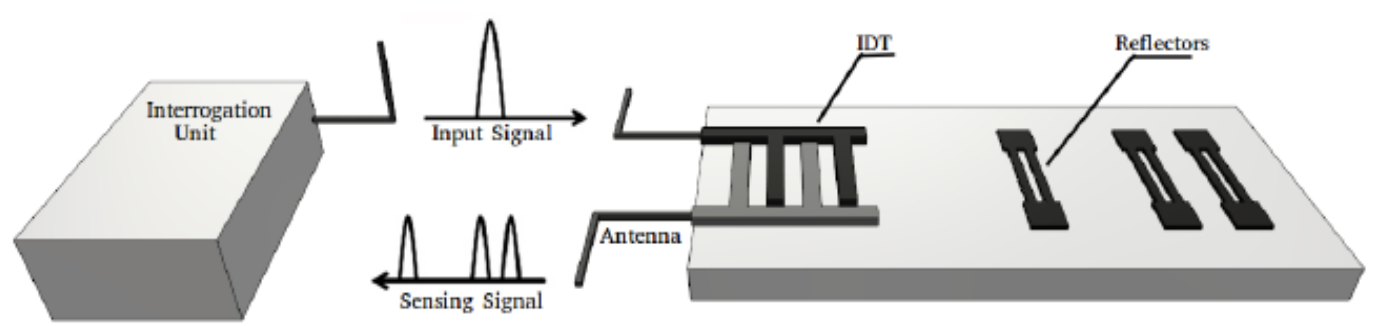

Figure 3.3 Wireless SAW sensor mode. 
For sensing purposes, changes in strain or temperature cause shifts in the acoustic wave velocity and/or the path length [25], enabling SAW devices to act as sensors for measuring temperature [26][27], pressure [26][28], stress, acceleration, strain [4][29], torque [17][30]. Some applications such tire pressure monitoring [31], gases detection [18], monitoring of humidity [32] and even pathogens were already implemented. In electronic applications, SAW devices have been mostly used as filters, rather than sensors, which are a more stablished commercial use.

SAW sensing devices are classified depending on their application as follows:

One Port Resonators: as shown in figure 3.4.a, consists of a single IDT generating and receiving the SAW with two grating reflectors, which reflect the SAW in phase at the center frequency, generating standing waves between these two reflectors.

Two Port Resonators: in this case, the structure is formed by two IDTs, as seen in figure 3.4.b. One IDT generates the SAW and the other receive it. In addition, two arrays of reflectors reflect the SAWs, confining them between the IDTs, inducing resonance.

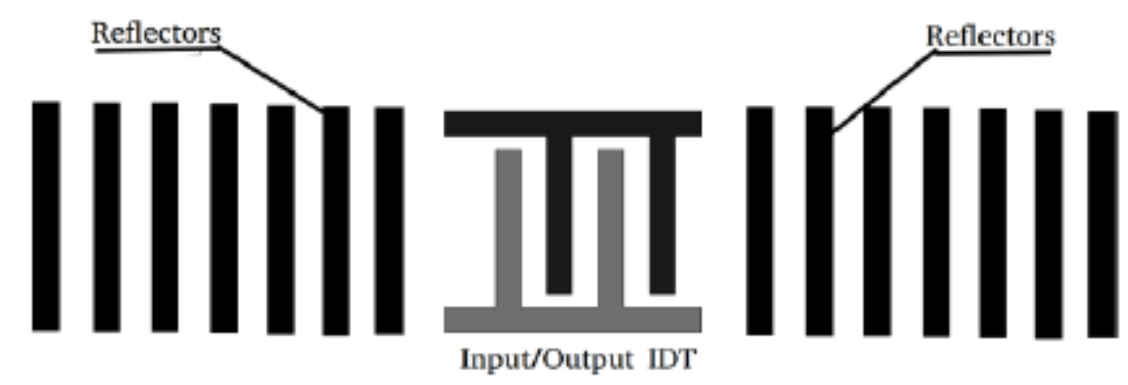

a.

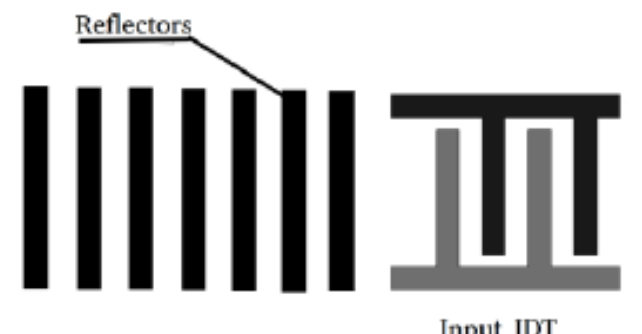

Input IDT

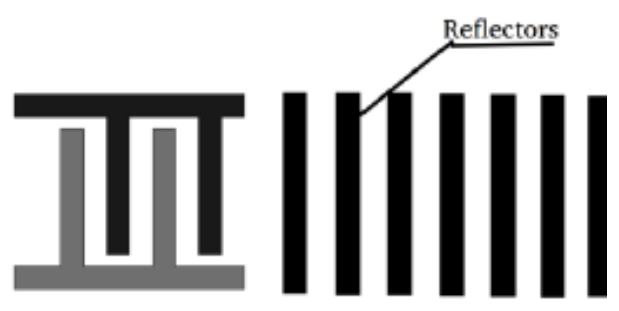

Output IDT

b.

Figure 3.4 SAW resonator configurations a. One-Port and b. Two-Port 
Delay Lines: in this device, the requested signal is separated from the response signal by a time difference or a path length. This is caused by the area between the IDTs often called the delay line, as shown in figure 3.5.

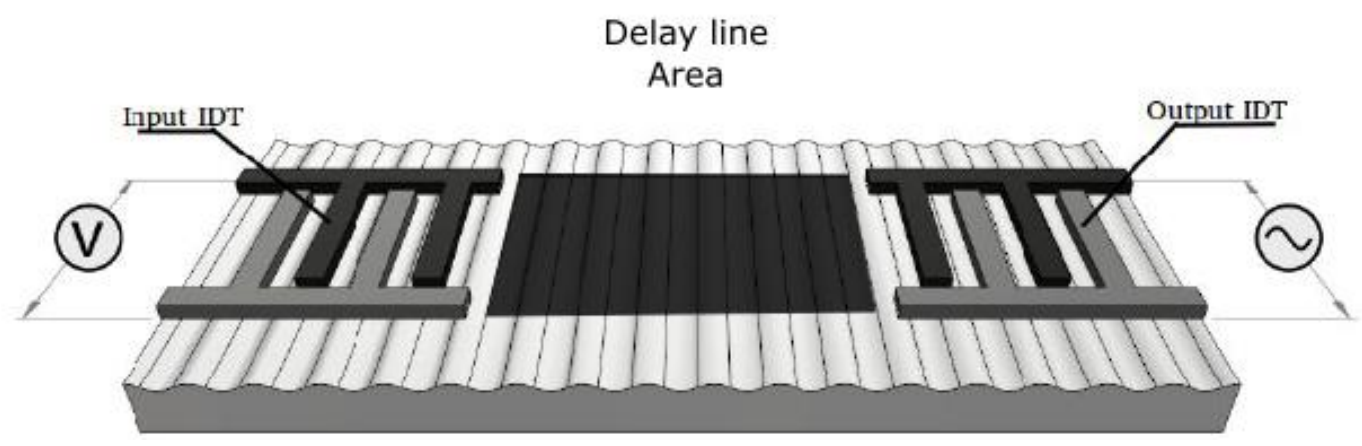

Figure 3.5 Two-port delay line configuration.

SAW resonates according to Bragg's frequency condition. This condition states that resonance will be strong when requirements $p=\lambda / 2$ and $N * r_{S}>1$ are achieved. In the expressions, $p$ is the finger pitch or periodicity, representing the distance from one finger to the next one in each comb group, and $\lambda$ is the wavelength, as shown in figure 3.6. $\mathrm{N}$ is the number of reflectors (typically around 200 or more) and $r_{s}$ is the reflection coefficient of one strip, which is about $2 \%$ [4]. The waves traveling in either directions are reflected constructively and destructively at two discrete frequencies forming a stopband.

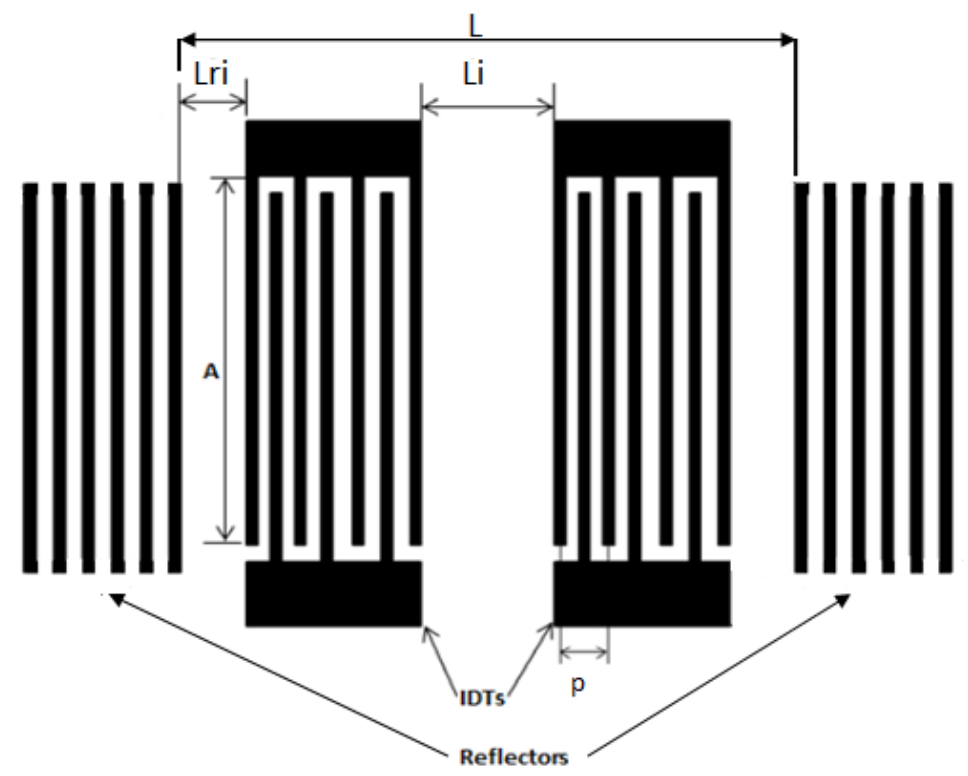

Figure 3.6. Dimension characteristics of an SAW two port resonators. 
The operating (resonant) frequency $f$ of the SAW device is chosen in relation to the wave velocity $v$ (that is a substrate dependent parameter). These parameters are plugged in equation 3.1 to compute the pitch value $p$ of the device IDT and consequently its wavelength $\lambda$.

$$
\begin{aligned}
& p=\frac{v}{f * S} \\
& \lambda=S * p
\end{aligned}
$$

where $S$ is the number of electrodes per period in the IDT that defines the total reflection and transduction of the device. Its common value is 2 because a greater number would reduce the inner reflection [23]. The acoustic aperture A is typically around 30-50 wavelengths (figure 3.6).

It is worth to point out that according to (Ramli, 2011), the optimum spacing between two IDT (figure 3.6), $L_{i}$, must be an integer number of half wavelength [22], as follows.

$$
L i=(n) \lambda / 2
$$

It is noted, as well, that the distance between reflectors, L, should be an integer number half wavelengths apart, as in equation 3.4.

$$
L=(n-1) \lambda / 2
$$

In addition, the distance between reflector grating and adjacent IDT, $L_{r i}$, will affect the transfer response of the resonator. The smaller the distance between them, the smaller the insertion loss produced [23].

Another important factor is the metallization ratio found in equation 3.5. It is given as a fraction of the electrode width, $a$, and the pitch, $p$, as can be seen in figure 3.7. This ratio is commonly equal to 0,5 to make the transducer highly reflective and also with a highly transduction per period. At last, the metal thickness or electrode height is given as a fraction of the wavelength in percentage as in equation 3.6.

$$
\begin{aligned}
& \text { Metallization ratio }=\frac{a}{p} \\
& \text { Metal thickness }=\frac{h}{\lambda} * 100
\end{aligned}
$$




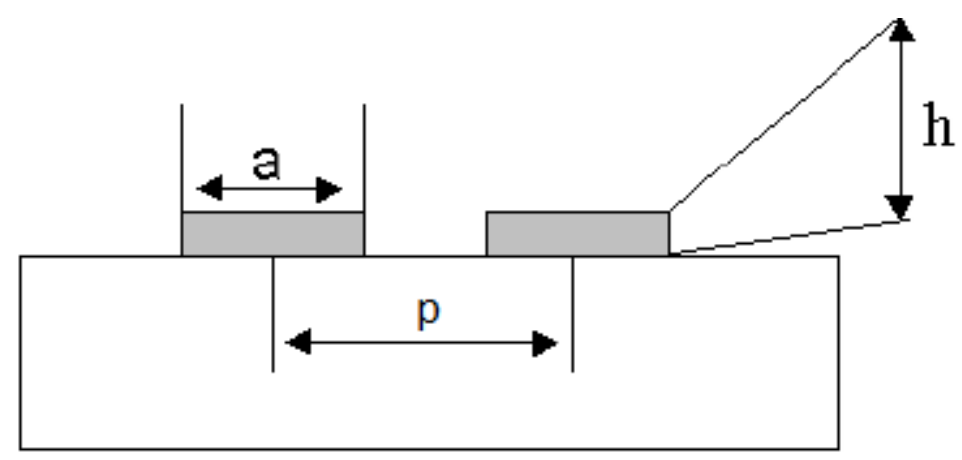

Figure 3.7 Finger dimensions of an SAW IDT

Regarding the number of electrodes in the reflectors array, a high number would minimize losses as they contain the reflected waves into a resonance cavity. The separation between reflectors array can be an integer number of half wavelength, generating standing waves and leading to a higher mechanical displacement.

\section{1 \\ Physics of surface acoustic waves devices}

In this section, theoretical description of acoustic waves is presented along with its mathematical formulation and generation in piezoelectric materials. In addition, comments on surface acoustics waves variations and its applications are made.

\subsection{1}

\section{Acoustic waves}

Transferring energy (supplied by an excitation source) in the form of oscillation or vibration, from one point to another is achieved through a wave propagating in space and time. These waves are elastic if propagate without causing permanent deformation to the solid and are characterized by their polarization. It is defined as the displacement direction of the particles in a particular coordinate system [17][23][33].

Waves are classified according to their polarization into two types, called longitudinal (compression) and transverse (shear) waves. In the first one, particle displacement occurs parallel to the propagation direction, and exhibits a volume change with propagation. In the second, particles move perpendicular to the propagation direction and do not exhibit volume change. As shown in figure 3.8. 
Longitudinal Waves

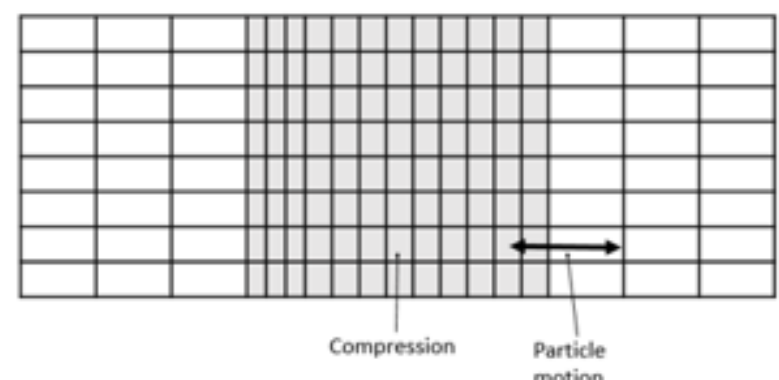

Shear Waves

Propagation

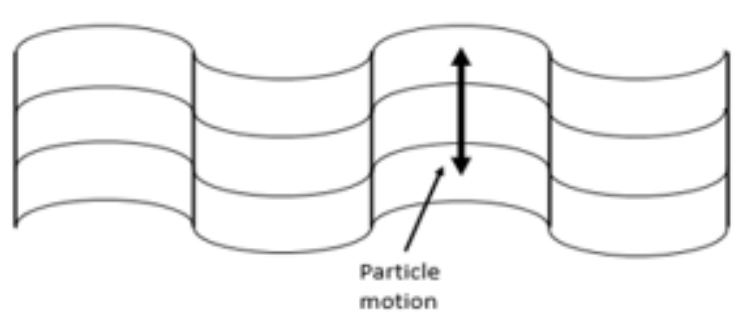

Figure 3.8 Longitudinal and Transverse (shear) waves

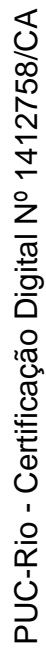

The illustration of a wave propagating in a medium is shown in figure 3.9, there two zones with different behaviors are presented. The wave spreading is due to a phenomenon called diffraction [33].

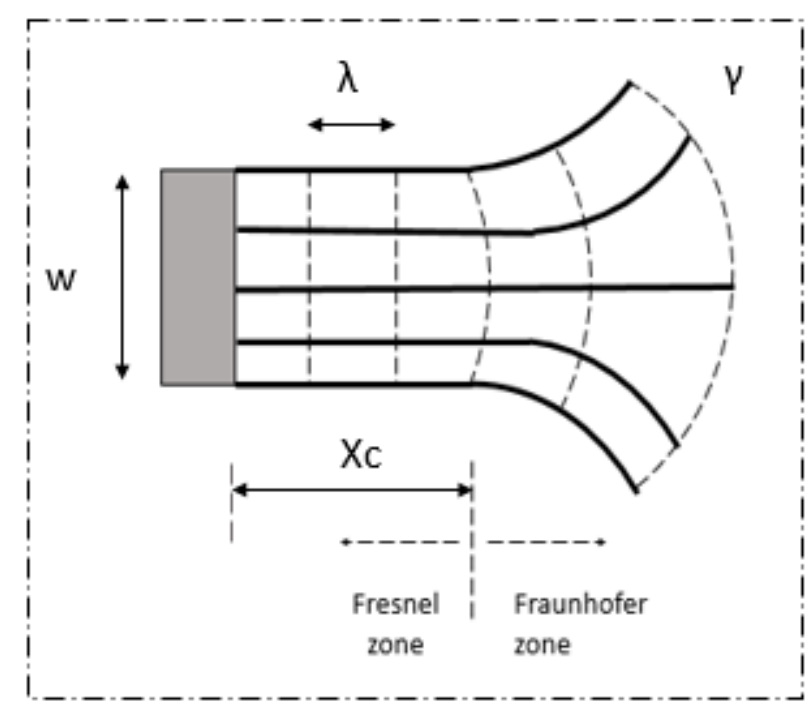

Figure 3.9 Propagation zones of a wave

In the first zone, defined as the Fresnel zone, wave propagates as a homogeneous plane wave, after which circular propagation occurs in the second zone named the Fraunhofer zone. The critical length $X c$ measured from the 
transducer, bounds those regions given by equation 3.7. This value is interesting for devices that require the homogenous propagation part of the wave.

$$
X c=(1+\gamma) w^{2} / \lambda
$$

where $w$ is the width of the transducer or the aperture, and $\gamma$ is a factor determined by the anisotropy of the media, being zero for isotropic materials.

\subsubsection{1}

\section{Wave equation}

To model the wave propagation, the proportional relation between mechanical stress $\mathrm{T}$ and strain $\mathrm{S}$ should be considered first, and is described by Hooke's Law for elastic deformations, expressed as a tensor equation.

$$
\begin{aligned}
& \{T\}=\{c\}:\{S\}=c: \frac{\partial U}{\partial X} \\
& \{S\}=\{s\}:\{T\}
\end{aligned}
$$

Where $c$ is the elastic stiffness tensor $\left[\mathrm{N} / \mathrm{m}^{2}\right], \mathrm{s}$ is the compliance tensor, and $\mathrm{U}$ are the displacements in the coordinate system directions.

Second, taking into account the Fundamental Law of Dynamics (Newton Law) given by:

$$
F=\frac{\partial T}{\partial X}
$$

Where $\mathrm{F}$ is the force density per unit volume, $T$ is the stress tensor and $X$ are axes of Cartesian coordinates.

Finally, it is possible to derive the wave equation for an isotropic material, expressed by equation 3.11 .

$$
\rho \frac{\partial^{2} U}{\partial t^{2}}=\frac{\partial T}{\partial X}=c: \frac{\partial^{2} U}{\partial X^{2}}=>\rho \omega^{2}=c k^{2}
$$

The term on the right of equation 3.11 is the dispersion relation of the wave. Where $\mathrm{k}$ is the wave vector (related to the wavelength by $k=2 \pi / \lambda$, this value 
gives the phase lag per unit length with propagation), $\omega$ is the angular frequency, and $\rho$ is the material density.

The size of equation 3.11 depends on the number of stiffness constants $c$ included in the matrix. They are normally 81 elements, and are not all independent, thus, their number can be reduced no matter if the material is isotropic or anisotropic. For an isotropic medium, only two elastic constants are independent, and are often called the Lamé constants $\lambda$ and $\mu$ (the last one is also known as the shear moduli) [23][34][35].

$$
\lambda=c_{11} \text { and } \mu=c_{44}
$$

One possible solution to this wave equation is a plane wave describe by

$$
U_{i}=A_{i} \exp [j(\omega t-k x)]
$$

where $A_{i}$ is the displacement amplitude and $\mathrm{t}$ is the time.

In each direction, three waves can propagate with different polarizations in the medium (but always perpendicular to each other); the solution above can be use to describe each one of the waves.

Also taking in to account that pure longitudinal and shear waves only exist for certain propagation directions, and in most of cases they are coupled, these three waves become one quasi-longitudinal and two quasi-shear waves. The quasi-longitudinal has a higher velocity than the other two. The two shear waves are differentiated through their phase velocity into the slow and fast shear waves, since the shear strain is dependent upon the direction of the motion.

In addition, as the wave velocity depends on the mechanical properties of the propagating medium, even if it is isotropic, the dispersion relation of the wave (being the ratio of angular frequency $\omega$ and wave vector $k$ ) gives the magnitude of their phase velocities, as shown below.

$$
\text { Longitudinal } \mathrm{V}_{\mathrm{L}}=\frac{\omega}{k}=\sqrt{\frac{c_{11}}{\rho}}, \text { Shear } \mathrm{V}_{\mathrm{s}}=\frac{\omega}{k}=\sqrt{\frac{c_{44}}{\rho}}
$$

where $\mathrm{C}_{11}$ and $\mathrm{C}_{44}$ are Lamè constants.

Common values found in the literature are around $V_{L}=6000 \mathrm{~m} / \mathrm{s}$ and $\mathrm{VS}=$ $3000 \mathrm{~m} / \mathrm{s}$ [34] [36] and, as it could be seen, the longitudinal waves are the fastest of all. 
Also is valid to mention that acoustic waves are of five order smaller in magnitude compared to electromagnetic waves, what make them very interesting to be used in many sensing applications [23]. It is convenient to transform these shear waves into two components with their polarization directions relative to substrate surface when dealing with surface acoustic waves. Thus one component is the Shear Horizontal (SH) wave with polarization parallel to the surface, and the other is the Shear Vertical (SV) wave with polarization perpendicular to that of the $\mathrm{SH}$ wave [33]. Which is the slow or the faster component, depends upon the anisotropy of the propagating medium.

\section{1 .2}

\section{The Piezo electricity effect}

Piezoelectricity is a phenomenon characterized by the production of electrical polarization in materials subjected to application of mechanical stress. The contrary effect is also possible, as application of electric fields to the material generates a deformation as a response. This effect is a coupling between elastic stresses and strains, with electric fields and electric displacements. This means that there is a relation between Stress-Strain-Charge that can be modeled through elasticity and Maxwell equations. These relations are described by the following equations:

$$
\begin{aligned}
& T=[C][S]-\left[e^{t}\right] E \\
& D=[e][S]+[\varepsilon] E
\end{aligned}
$$

where $S$ is the strain, $e$ is the matrix of piezoelectric constants, $E$ is the electric field in reference axes directions, $D$ is the electric displacement, $\varepsilon$ is the permittivity matrix and $C$ are the stiffness matrix.

Through some assumptions like that magnetic fields are disregarded (because they are not important in acoustic wave propagations), and that the piezoelectric materials are almost perfect insulators [23], the Maxwell's equation reduces to

$$
\nabla \cdot D=0
$$


Since acoustic wave velocities are about five orders of magnitude smaller than electromagnetic waves, they can be considered as quasi-static, leading to express the electric field as the negative gradient of the scalar potential $\varphi$.

$$
E=-\nabla \varphi
$$

However, due to the complexity of the wave equations coupled with the piezoelectric effects, solutions are found only by numerical methods. So, approximations can be given in terms of displacement and the potential, as expressed below.

$$
\begin{gathered}
\boldsymbol{u}=\boldsymbol{u}_{0} \exp [j(\omega t-k \cdot x)], \\
\varphi=\varphi_{0} \exp [j(\omega t-k \cdot x)]
\end{gathered}
$$

Where $\omega$ is the frequency, and $k$ is wave vector.

\subsection{3}

\section{Piezoelectric crystals}

For generation and detection of acoustic waves, piezoelectric materials are used. Piezoelectric materials are anisotropic (their internal structure lacks a center of symmetry), and the properties of acoustic waves vary upon the directions of propagation and/or polarization with respect to the internal crystal orientation [34][37]. Therefore, detailed information about crystal characteristics are presented below.

\subsubsection{1}

\section{Crystal structure}

Piezoelectric materials exhibit absence of mirror symmetry that can be observed in the crystal form. They have several faces in their solid structure, which in turn, can be used to identify them. For instance, quartz crystals faces are shown in figure 3.10, and the difference between a left or right-handed quartz can be identified. Therefore, due to the atoms in this crystal are arranged in parallel corkscrew-like chains or helices, the internal molecular structure of the quartz crystal cannot be mirrored. As helix lacks mirror symmetry, it is always either leftor right-handed. 
In addition, figure 3.11 depicts the cross-sections of 3 quartz crystals, showing that the angles between corresponding crystal faces of the same mineral never change no matter its shape or if they are distorted.

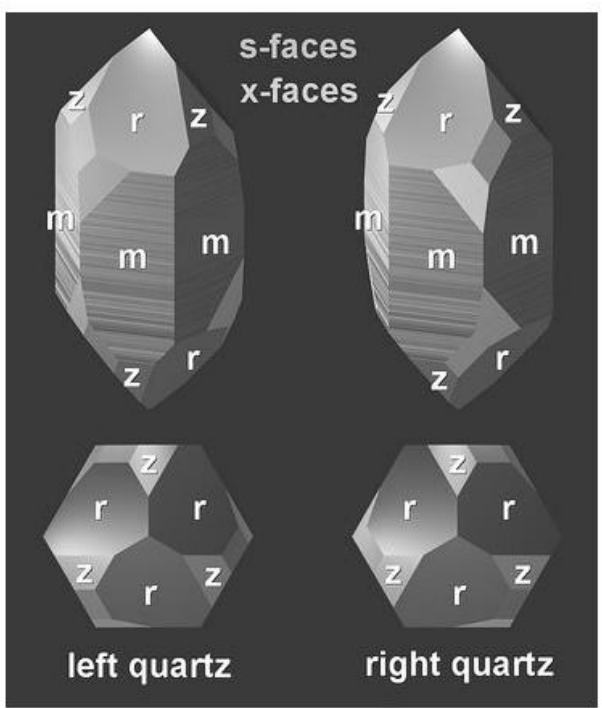

Figure 3.10 Left and right crystal quartz
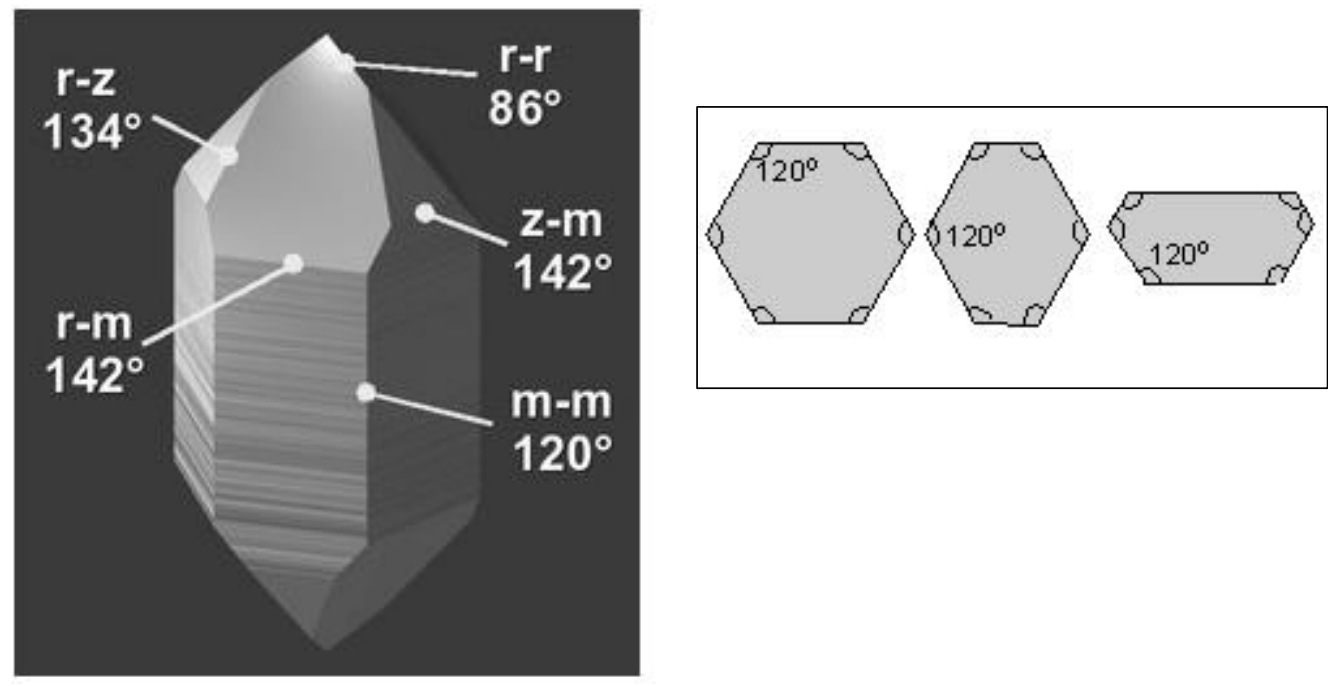

Figure 3.11 Angle between faces of a crystal never change even when distorted.

All crystals have an internal arrangement with an atomic structure organized as symmetrically distributed nodes and groups of atoms attached to each node depicted in figure 3.12 [37]. This leads to a 3D lattice repeated periodically that grows forming smooth planar boundaries up to the crystal surface, which finally reflects that internal node symmetry. 


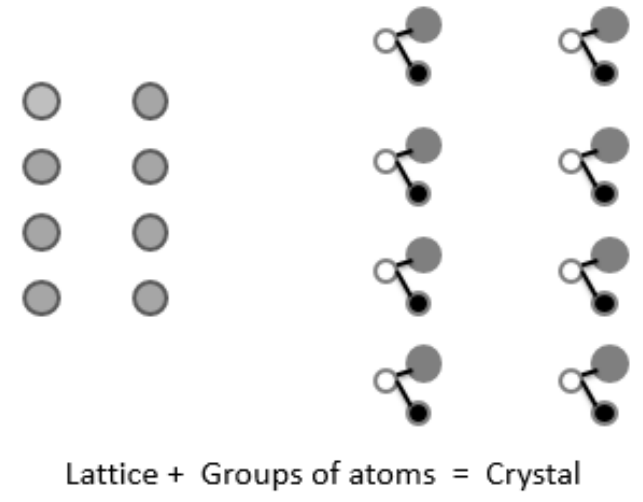

Figure 3.12 Crystal lattice structure

The derivation of the compliance and stiffness matrices depends on the microscopic properties of the material. Symmetry operations, such as the axis coordinate transformations of the inner lattice structure can reduce the number of coefficient of these matrices and even transform the crystal lattice. Taking into account that a point can be used to describe stress and strain fields [34][36], operations like rotations, reflections, inversions, and the combination between them, applied over that selected point, are enough to obtain the symmetric properties of the material.

In the following analysis, rotation operations are performed and defined as the smallest rotation under which the lattice is symmetric. For instance, an n-fold rotation symmetry type is defined as a minimum rotation angle of $2 \pi / n$ that the object once rotated, takes to be restored to its original geometry or position. These rotations can only be of the type 2-fold, 3-fold, 4-fold and 6-fold occurring in a crystal lattice, as shown in figure 3.13.a, because the other rotations, such as 5 fold or 8-fold cannot fulfill the entire space due to internal geometric restrictions. Examples of these restrictions can be seen in figure 3.13.b.

a.

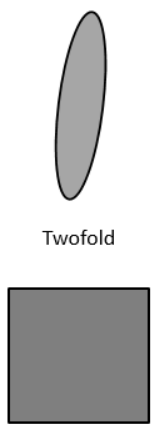

Fourfold

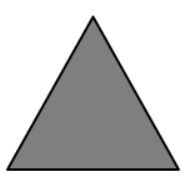

Threefold

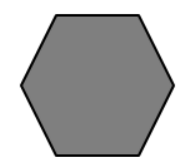

Sixfold b.

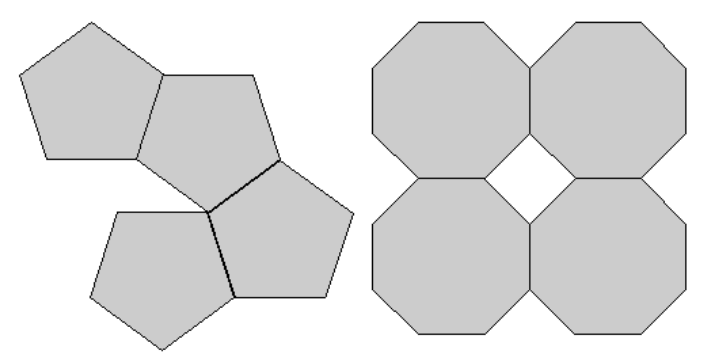


Figure 3.13. Rotation operations a. Types, b. Restrictions

Concerning a $3-$ fold rotation, it is formed by a $2 \pi / 3=120^{\circ}$ angle described by a small triangle identified with number 3 , as shown in figure 3.14. Another repetition of the rotation leads to $4 \pi / 3=240^{\circ}$, symbolized by $3^{2}$. A third repetition leads to a $6 \pi / 3=360^{\circ}$ angle or $3^{3}=1$ symbol, which means that the rotation has returned to its original position, as shown also in figure 3.14.

All this operation in crystallography is called a group, and each crystal symmetry group is called a class. Various classes together form a system that have certain physical properties in common [34], [37].

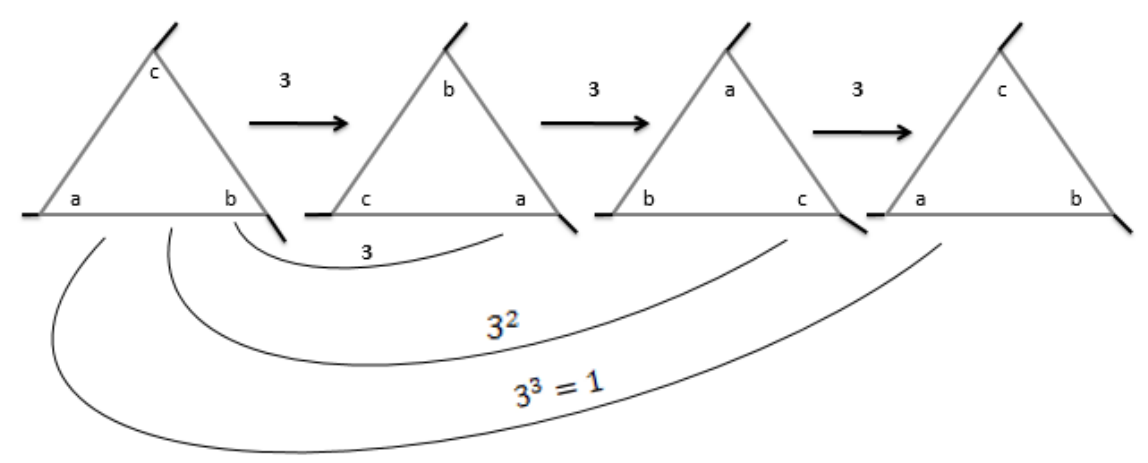

Figure 3.14 Three Fold rotation representation

Another type of operations are mirror or reflections made over planes. They are denoted by the symbols $m$ or $\bar{m}$ if the mirror plane is normal to the page or laying on it, respectively. Also, inversion or reflection operations over a point uses the symbol $\bar{I}$.

Crystal classification utilizes 7 crystal systems characterized by the geometric form of the cell, according to the point of symmetry that the lattice exhibits. These cells form vary from a cube to a parallelepiped, and are normally called the fourteen Bravais lattices, as shown in figure 3.15.

In the image, $\mathrm{P}$ refers to the primitive lattice, which is the base or minimum structure and different structures can be obtained with the addition of extra nodes. For instance, they can be located at the center of the cell in the case of body centered (I), or at the center of all faces, (F) called face centered, or even with nodes located at center of two opposite faces called opposite face centered $(\mathrm{C})$. 


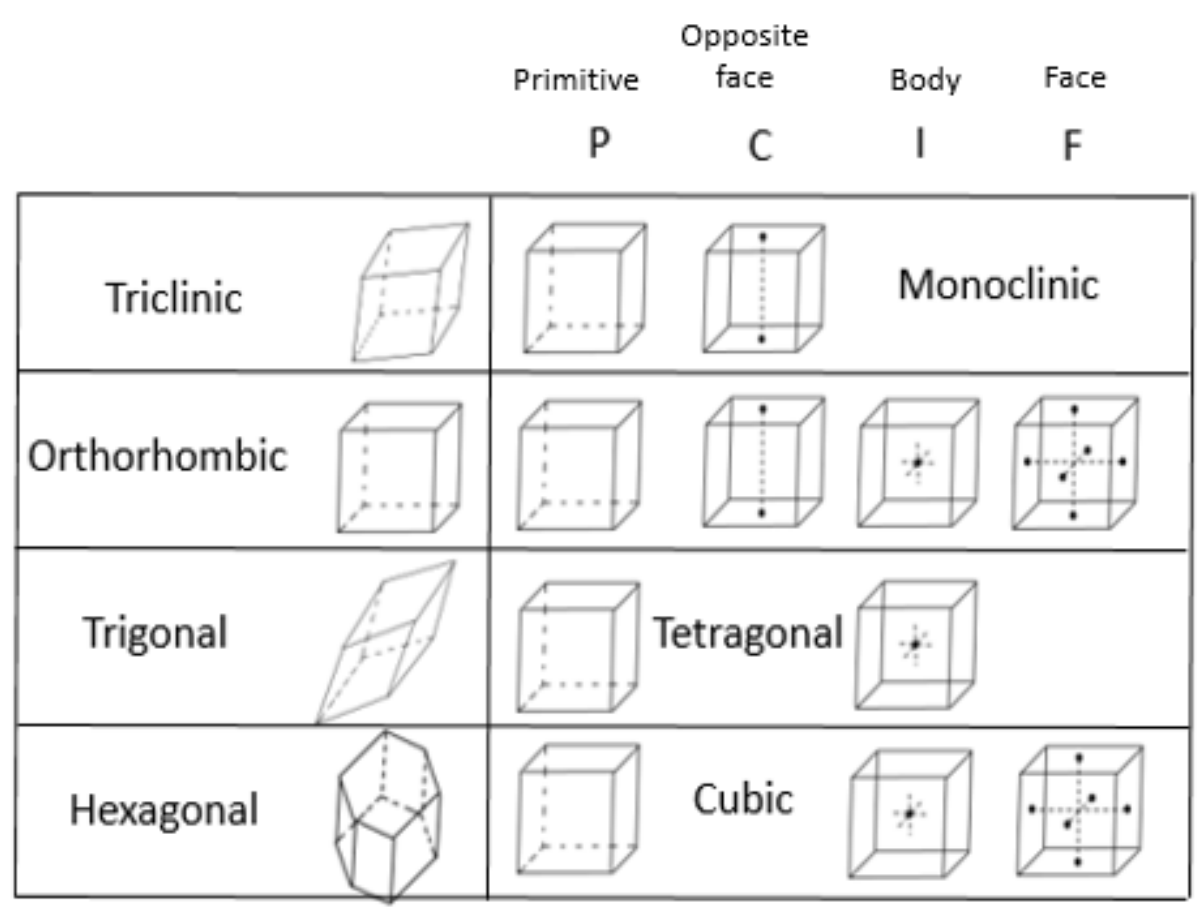

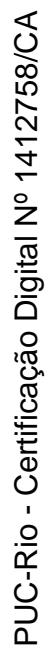

Figure 3.15 Crystal systems

Finally, the relation of structures classes with the symmetry operations defined above, are shown in the table 3.1. It is worth to point out that 20 out of the 32 crystal classes are potentially piezoelectric.

Table 3.1 Crystal system and classes

\begin{tabular}{|c|c|c|c|}
\hline System & Class & System & Class \\
\hline Monoclinic & $\frac{1}{1}, 2, \mathrm{~m}, \frac{2}{\mathrm{~m}}$ & Trigonal & $\frac{3}{3}, 32,3 \mathrm{~m}, \overline{3} \mathrm{~m}$ \\
\hline Orthorhombic & $222, \mathrm{~mm} 2, \mathrm{mmm}$ & Hexagonal & $6 \mathrm{~m}, \frac{6}{\mathrm{~m}}, \frac{6}{\mathrm{mmm}}$ \\
\hline Tetragonal & $4, \overline{4}, \frac{4}{\mathrm{~m}}, \frac{4}{\mathrm{mmm}}, 4 \mathrm{~mm}$ & Cubic & $\begin{array}{c}23,3 \mathrm{~m}, \mathrm{~m} 3,432, \\
\mathrm{~m} 3 \mathrm{~m}\end{array}$ \\
\hline Isotropic & & & \\
\hline
\end{tabular}

\subsubsection{2}

\section{Crystal cuts}

Piezoelectric substrates are commercialized in thin wafers that have been cut at a particular angle respect to the crystal axes; they are usually defined as follows: 
Internal structure of crystalline materials are described by axes with symbols $X, Y, Z$. Axis directions follow a convention related to the crystal lattice, which defines the surface orientation and the wave propagation direction. Specifically, the normal direction to the surface is defined as $x_{3}$ followed by the propagation direction $x_{1}$ and in addition, this $x_{3}$ direction is also referred as the substrate cut type. For instance to show how this definition works, the $Y-Z$ lithium niobate substrate specification means that, $x_{3}$ is parallel to the crystal $Y$ axis and $x_{1}$ is parallel to the $Z$ axis, but also means that it is a crystal with $Y$-cut.

Unfortunately, there are two standards in the literature used for reference axes, as shown in figure 3.16 and material properties take different forms within them. The IEEE 1978 Standard and the IRE 1949 Standard, they are not always specified and none of them have shown certain preference by literature. In this figure 3.16 Quartz AT cut defined as a y rotated cut $35,25^{\circ}$ around $x$ axe by the 1949 IRE and as a y rotated cut $35,25^{\circ}$ around $x$ axe repect to $z$ axe by the IEEE 1978.

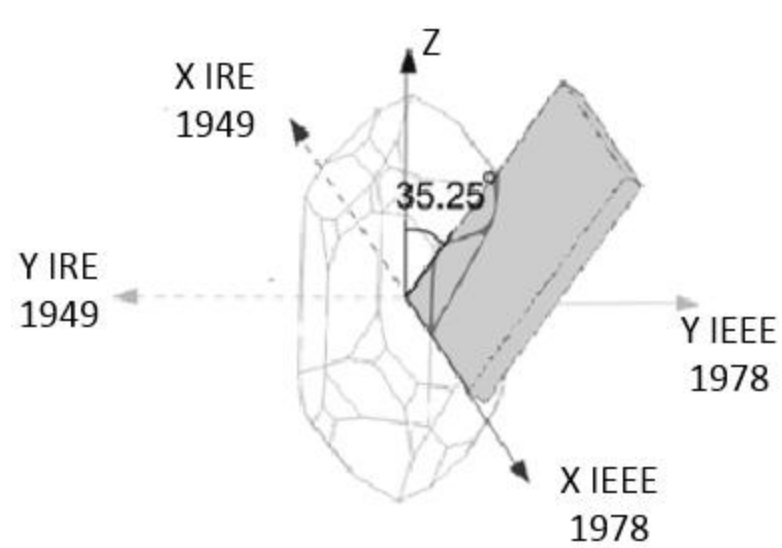

Figure 3.16 Crystal standards definitions. @Comsol.

As an example of a cut, Quartz belongs to the trigonal trapezohedral class (32) of the rhombohedral subsystem, it has one axis of three-fold symmetry and three axes of two-fold symmetry perpendicular to the former, as shown in figure 3.17. $X$ is one of the two-fold symmetry axes being the "electrical axis" and the other axis $Z$ is of three-fold symmetry [38]. 

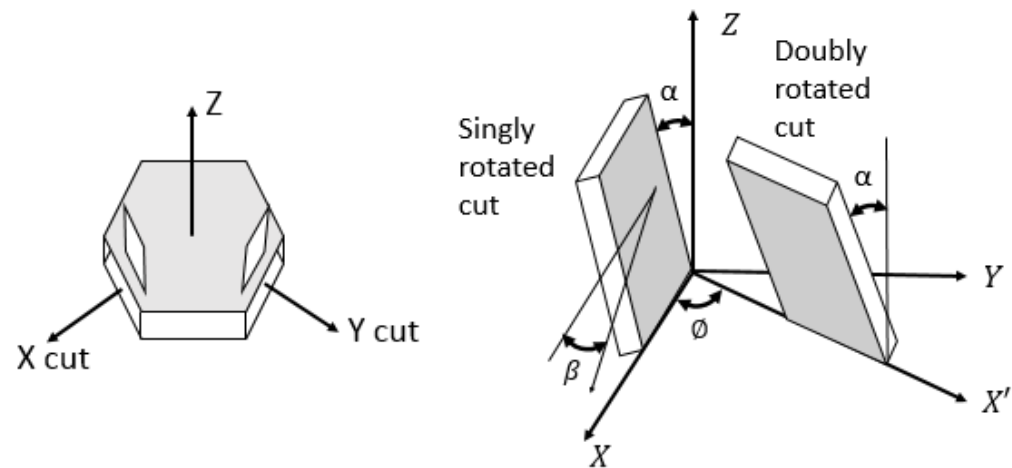

Figure 3.17. Quartz crystal characteristics (Avramescu, 2009).

With a substrate having $Y$ wafer cut, if an electrical field is applied along $X$ axis, Rayleigh waves are propagated along this axis. Instead, if the field is applied to the $\mathrm{Y}$-axis then shear waves $\mathrm{SH}$ are generated. This points out, the importance of some characteristics derive from choosing the right piezoelectric substrate cut for a specific application, for instance some SAW cuts are shown in the table 3.2:

Table 3.2 Piezoelectric substrate properties [38]

\begin{tabular}{|c|c|c|c|c|}
\hline & $\begin{array}{c}\text { Y-Z } \\
\text { Lithium } \\
\text { Niobate }\end{array}$ & $\begin{array}{c}\mathbf{1 2 8}^{\circ} \mathbf{~ Y - X} \\
\text { Lithium } \\
\text { Niobate }\end{array}$ & $\begin{array}{c}\mathbf{1 3 1 . 5}^{\circ} \mathbf{Y - X} \\
\text { Lithium } \\
\text { Niobate }\end{array}$ & $\begin{array}{c}\text { Lithium } \\
\text { Niobate }\end{array}$ \\
\hline $\begin{array}{c}\text { Elect. Mech. } \\
\left.\text { Coupling \% [K }{ }^{2}\right] \\
\text { SAW }\end{array}$ & 4.8 & 5.3 & 5.6 & 5 \\
\hline $\begin{array}{c}\text { Phase velocity } \\
\text { [m/s] } \\
\text { SAW }\end{array}$ & 3488 & 3992 & 4000 & 3992 \\
\hline
\end{tabular}

\section{2}

\section{Wave modes}

Since the acoustic wave power spreads uniformly in all directions with equal speed into the substrate in BAW (Bulk Acoustic Waves), in some points this power decays with depth because of the law of energy conservation [33], as can be seen in figure 3.18. Inside of this zone, both longitudinal and shear vertical waves are present and coupled each other to compose an eigenmode called surface acoustic 
waves SAW or Rayleigh waves, in honor to Lord Rayleigh who described them first in 1885 .
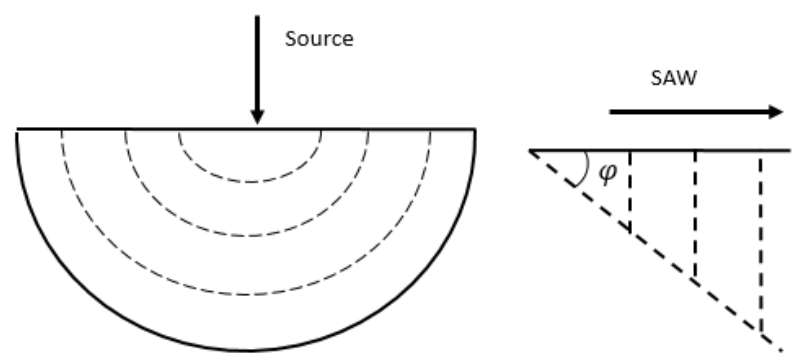

Figure 3.18 Wave power spreading. (Hashimoto, 2000)

This wave has an angle respect to the surface, called the critical angle $\Phi c$ and is the limit from where there are only bulk waves present.

$$
\Phi c=\cos -1(V s / V b)
$$

Where $V_{s}$ and $V_{b}$ are the phase velocities of surface and bulk acoustic waves.

SAW are composed by a shear vertical and longitudinal waves because the propagation solution of this surface wave needs to satisfy the no traction condition on the surface [35]. Therefore, as shown in figure 3.19, waves propagate in $x_{1}$ and their wavefront parallel to $x_{2}$ with no variation in this direction. In addition, $x_{1}$ and $x_{3}$ forms the sagittal plane where the wave is contained with particle displacements of an elliptical shape. SAW distinctly do not penetrate more than one wavelength $\lambda$, as the amplitude decays exponentially away from the surface, as shown in figure 3.20 .

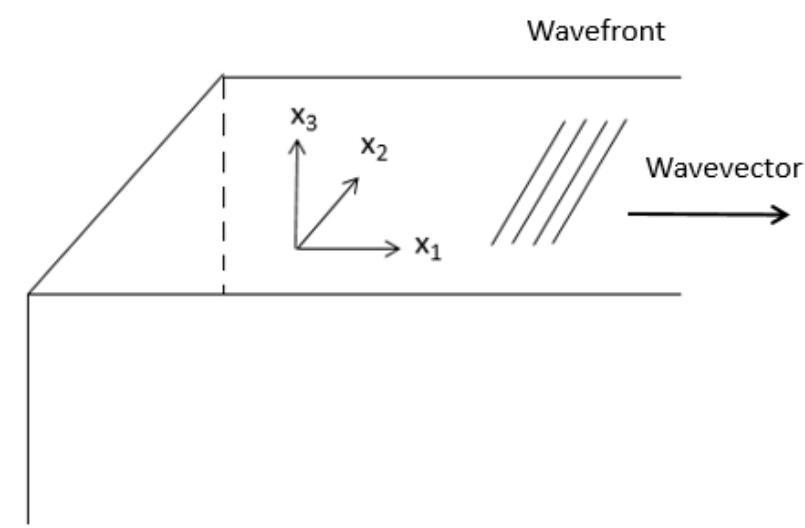

Figure 3.19. Surface acoustic wave 


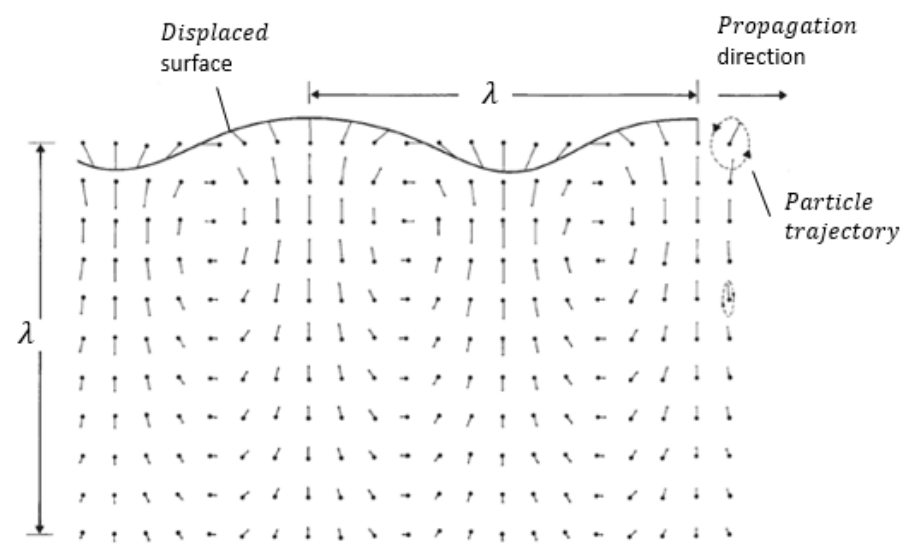

Figure 3.20. Particle displacement (Morgan, 2007)

This surface acoustic wave velocity has to be less than plane wave velocities (longitudinal, fast shear and slow shear), in fact, it has to be less than the slow shear wave velocity as it is the lowest of all plane waves. This condition is due to the wave vectors must not have a real $x_{3}$ component as a surface wave, but usually they are quite close to each other [35]. Some velocity values are shown in table 3.3.

However, in certain isolated directions the Rayleigh velocity exceeds the velocity of the slowest shear wave (but still being less than the fast shear), what leads to a special SAW called a Pseudo-SAW, which propagates without attenuation in the medium [23][35]. This wave occurs when its displacement is perpendicular to the displacement of the slow shear wave and its partial component is eliminated. In the propagation of waves with a different direction than the mentioned above, this partial wave corresponding to the slow shear cannot be neglected. This partial component carries some energy away from the surface, causing small attenuations or energy leakage [35], then taking the name of Leaky surface wave. In Figure 3.21 is shown different waves characteristic velocities.

Table 3.3 SAW properties and applications, Collin Campbell [39]

\begin{tabular}{|c|c|c|c|c|c|c|}
\hline Material & $\begin{array}{c}\text { Crystal } \\
\text { Cut }\end{array}$ & $\begin{array}{c}\text { SAW } \\
\text { Axis }\end{array}$ & $\begin{array}{c}\text { Velocity } \\
{[\mathrm{m} / \mathrm{s}]}\end{array}$ & $\begin{array}{c}\mathrm{K}^{2} \\
{[\%]}\end{array}$ & $\begin{array}{c}\text { Temperature } \\
\text { Coef. De } \\
\text { Delay }\end{array}$ & Applications \\
\hline Quartz & ST & $\mathrm{X}$ & 3158 & 0.11 & 0 & $\begin{array}{c}\text { Oscillators, } \\
\text { Filters }\end{array}$ \\
\hline
\end{tabular}




\begin{tabular}{|c|c|c|c|c|c|c|}
\hline $\mathrm{LiNbO}_{3}$ & $\mathrm{Y}$ & $\mathrm{Z}$ & 3488 & 4.5 & +94 & $\begin{array}{c}\text { Wideband } \\
\text { Filters }\end{array}$ \\
\hline $\mathrm{LiNbO}_{3}$ & $128^{\circ}$ & $\mathrm{X}$ & 3992 & 5.3 & +75 & $\begin{array}{c}\text { Wideband } \\
\text { Filters }\end{array}$ \\
\hline $\mathrm{Bi}_{12} \mathrm{GeO}_{20}$ & 110 & 001 & 1681 & 1.4 & +120 & $\begin{array}{c}\text { Long Delay } \\
\text { times }\end{array}$ \\
\hline $\mathrm{LiTaO}_{3}$ & $\mathrm{Y}$ & $\mathrm{Z}$ & 3230 & 0.72 & +35 & \begin{tabular}{c} 
Oscillators \\
\hline $\mathrm{GaAs}$
\end{tabular} \\
\hline
\end{tabular}

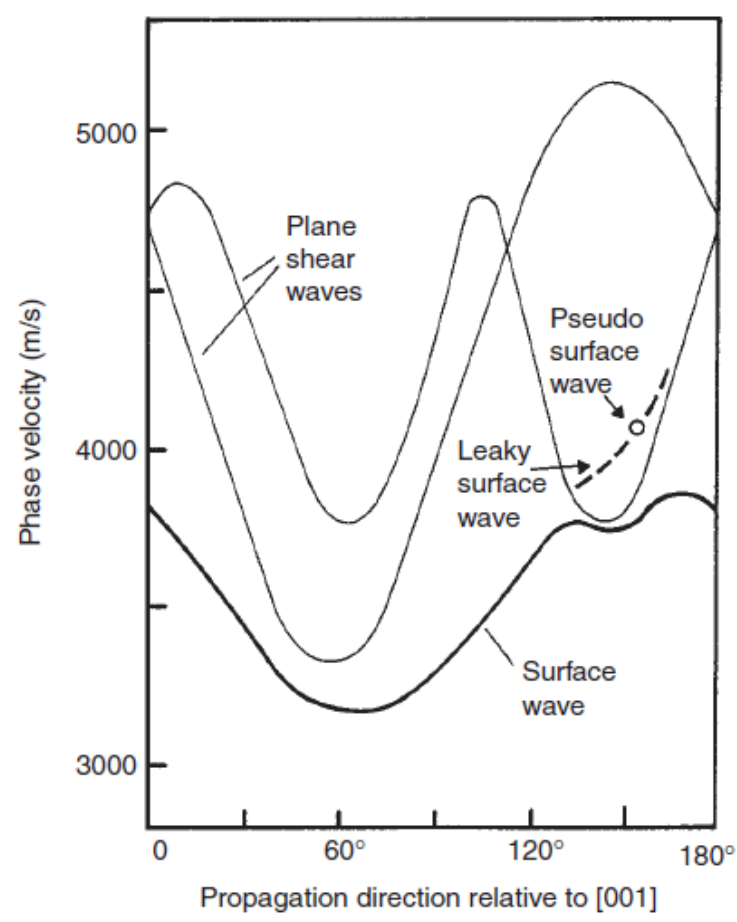

Figure 3.21 Acoustic wave modes relations.

Another different type of wave is called the Bleustein-Gulyaev wave, which exists if the sagittal plane is normal to an even-order axis (2-, 4- or 6-fold) of the crystal. Consequently its displacement is also normal to this plane, associated to an electric field that has bounded it to the surface [35].

Other Rayleigh related waves (Sagittal plane polarization) are the Lamb waves, which propagates in plates considered as being two Rayleigh waves propagating on each side of the plate. If the plate is thicker than two wavelengths, 
then two free Rayleigh waves propagate with displacements confined to this sagittal plane [40].

In addition, Love waves are guided acoustic waves that propagate on a thin layer deposited over the substrate, its energy is concentrated there and their displacements are normal to the sagittal plane. However, in this case, the partial waves are only shear waves. With this particle polarization only existing in the shear horizontal SH direction, almost no energy is coupled into liquids for the top layer, thus Love waves are also suitable for detection in liquids. Device performance depend now on the guiding layer rather than on IDT structures and substrate characteristics, as it is in SAW devices. Therefore, Love waves can be regarded as modified forms of the $\mathrm{SH}$ plane wave, where the presence of a layer with low acoustic velocity converts the plane wave into a surface wave causing dispersion.

Acoustic plate mode APM, are shear horizontal waves with particle displacement predominantly parallel to the substrate surface although not contained only on it. They are distributed throughout the substrate and normal to the direction of propagation.

Surface transverse wave STW are also shear horizontally polarized waves, trapped at the surface through a periodic surface perturbation structure. For example, a metallic grating which slows down the wave, showing a higher mass sensitivity compared to APM for gravimetric applications.

Some applications of those acoustic modes are shown in the table 3.4 for comparison with their own characteristics [41].

Table 3.4 Different Wave types performance comparison

\begin{tabular}{|c|c|c|c|}
\hline Wave type & $\begin{array}{c}\text { Gas or Liquid } \\
\text { operation }\end{array}$ & Robustness & Application \\
\hline Rayleigh & $\mathrm{G}$ & High & Gas, voltage \\
\hline APM & $\mathrm{G}+\mathrm{L}$ & Moderate & $\begin{array}{c}\text { Gas, biochemical, } \\
\text { viscosity }\end{array}$ \\
\hline Lamb & $\mathrm{G}+\mathrm{L}$ & Low & $\begin{array}{c}\text { Gas, biochemical, } \\
\text { moderate } \\
\text { density, sound, speed }\end{array}$ \\
\hline Love & $\mathrm{G}+\mathrm{L}$ & High & Gas, biochemical \\
\hline G+L & High & $\begin{array}{r}\text { Gas, biochemical, } \\
\text { viscosity }\end{array}$ \\
\hline
\end{tabular}




\section{Experiments}

As part of an environmental monitoring tool, a humidity sensor system was developed. It consists of two SAW resonators, chosen due to their favorable characteristics for gravimetric (mass) measurements when compared to others acoustic waves technologies and also their commercial availability, as shown in the table 4.1. In the system one resonator is denominated the reference SAW and the other the humidity SAW resonator. The only difference between the two resonators is that the humidity SAW resonator has a Polyvinyl Alcohol (PVA) film deposited on it. The operation principle of the humidity sensor system is to correlate the difference between the frequencies of the resonators with the surrounding humidity. In the next Section, details of the PVA film preparation and the electronic circuit development are to be described.

Table 4.1 Different acoustic wave sensors properties

\begin{tabular}{|c|c|c|c|c|c|c|}
\hline \multirow{2}{*}{ Sensor type } & FRO & \multirow{2}{*}{$\mathbf{S}_{\mathbf{m}}$} & \multicolumn{3}{|c|}{ Examples } & \multirow{2}{*}{ OL } \\
\cline { 4 - 6 } & & & FO & FN & $\mathbf{S} / \mathbf{N}$ & \\
\hline TSM quartz & $5-30$ & $12-70$ & 10 & 0.2 & 110 & Yes \\
\hline Thin-fillm BAW & $900-1000$ & $400-700$ & & & & No \\
\hline SAW & $30-500$ & $100-500$ & 160 & 2 & 100 & No \\
\hline SH-APM & $20-200$ & $20-40$ & 100 & 4 & 5 & Yes \\
\hline STW & $100-200$ & $100-200$ & & & & Yes \\
\hline LW & $100-200$ & $150-500$ & 110 & 2 & 125 & Yes \\
\hline FPW & $5-20$ & $200-1000$ & 5 & 1 & 450 & Yes \\
\hline
\end{tabular}

With FRO being the frequency range of operation $[\mathrm{MHz}], \mathrm{Sm}$ is the mass sensitivity, $\mathrm{FO}$ is the frequency of operation $[\mathrm{MHz}], \mathrm{FN}$ is the frequency noise $[\mathrm{Hz}], \mathrm{S} / \mathrm{N}$ is the sensitivity to noise ratio and $\mathrm{OL}$ is the operation in liquid [42].

\section{1}

\section{Sensing Polymer}

The fundamental part of our sensor is the polymer layer deposited on top of the SAW resonator, being the medium through which the analyte is detected and measured. The properties of the polymer layer vary with absorption or adsorption 
of the analyte that cause changes in the device operation. These polymer layers are typically used in both gas sensing and bio-sensing applications.

\subsection{1}

\section{Diffusion and Fick's Law}

Diffusion can be defined as a process by which some material molecules moves from a high concentration zone to a lower concentration zone and it is a critical mechanism of polymer/analyte-based gas sensing in acoustic sensors. This phenomenon is normally described by Fick's first law of diffusion for a onedimensional system as follows:

$$
J=-D_{F} * \frac{d^{\Phi}}{d z}
$$

Where $J$ is the diffusion flux in $\frac{\mathrm{mol} * \mathrm{~m} 2}{\mathrm{~s}}, \mathrm{D}_{\mathrm{F}}$ is the Fickian diffusion coefficient in $\frac{\mathrm{m} 2}{\mathrm{~s}}$,

$\phi$ for ideal mixtures is the concentration in $\frac{\mathrm{mol}}{\mathrm{m} 3}$ that is a function of $z$, being the depth of penetration in the polymer.

The polymer thickness determines the total mass response derived from analyte absorption. Therefore, with a rate of diffusion determined by the diffusion coefficient, it is observed that thicker polymers will require more time to reach equilibrium. This would reduce the time response rate of the sensor but would increase the sensitivity, as more analyte enters the polymer.

In conclusion, sensors with thicker polymer layers demonstrate greater overall response when exposed to a variation in analyte concentration, but they respond much slower as a large amount of analyte was required to reach equilibrium. It is worth to point out that this diffusion coefficient is higher if polymer and analyte present similar characteristics, as hydrogen bonds, polar bonds and Van der Waals forces [43].

Taking those conditions into account, one port SAW resonators coated with a polymer layer have been tested to analyze the SAW performance in gas sensing applications [44][45][46]. Specifically several water vapor / PVA experiments were conducted as PVA is known to absorb water vapor and has been used extensively in water and vapor sensing [47][32], [48], [49]. In addition, PVA swelling mechanism and corresponding changes of volume and density as functions of relative humidity are well studied [50][51][52]. 
Konidari et al. studied how the stiffness of PVA changes as a function of humidity showing the relationship between tensile strength, Young's modulus, and glass transition temperature of PVA films being exposed to different humidity values [53]. But in this study only density variations are going to be analyzed as explained in the section 4.5 .

\subsection{2}

\section{Preparation of Poly(vinyl Alcohol) Films}

As a part of the humidity sensor, an aqueous solution containing $5.6 \mathrm{wt} \%$ of poly (vinyl alcohol), also known as PVA, was prepared by dissolving the polymer in distilled water. Initially, the PVA polymer (average molecular weight, $\mathrm{Mw}=130,000$, Aldrich) was dissolved by magnetic stirring a whole night at room temperature until a homogeneous solution was obtained. In order to eliminate the air bubbles that were entrapped in the solution during mixing, a vacuum degassing process was used. To achieve this, the container with the solution was placed in a vacuum chamber connected to a vacuum pump, which was kept inside to remove the air in the solution at $-760 \mathrm{mmHg}$. After degassing, the solution was used to prepare the humidity SAW sensors, and also used to prepare film samples to characterize the mechanical properties of this PVA solution. In ¡Error! No se e ncuentra el origen de la referencia. is shown the schematic diagram of the PVA film preparation process.

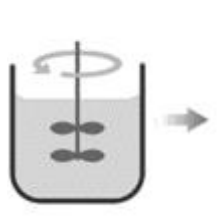

Stirrer

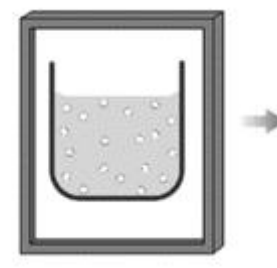

Vacuum

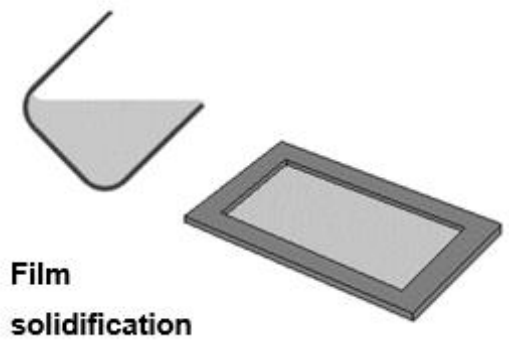

Figure 4.1 PVA film preparation in molds

\subsection{3}

\section{PVA deposition}

To obtain the humidity system, two single-port SAW resonators were used but only one have a PVA film deposited on it. The deposition of the film involves a process beginning with the carefully removal of the top of the metal packaging 
in order to access the SAW structure inside the package, see figure 4.2. Once the SAW is uncovered, a $20 \mu \mathrm{l}$ drop of the aqueous PVA solution described in the 4.1.2 section was placed over the SAW device. After that, a homogeneous film was obtained by spinning the device at $3000 \mathrm{rpm}$ during $20 \mathrm{~s}$, and then cured at $60^{\circ} \mathrm{C}$ for $30 \mathrm{~min}$.
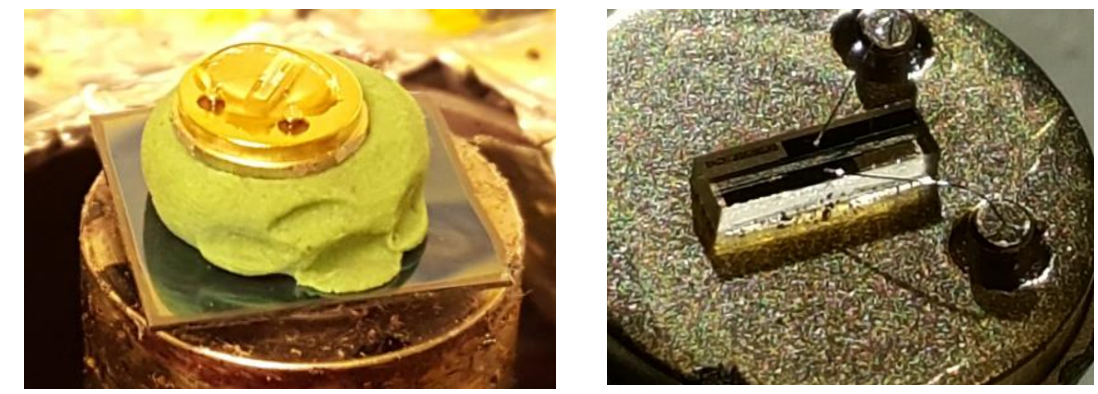

Figure 4.2 The SAW resonator unit without packaging type TO-39

The final film thickness was of approximately $500 \mathrm{~nm}$. This thickness could be controlled by changing the rotation rate. figure 4.3 ¡Error! No se encuentra el origen de la referencia.shows the SAW resonator before and after the film deposition and the resulting PVA film covered completely the SAW resonator, including the reflectors and interdigital transducer (IDT).
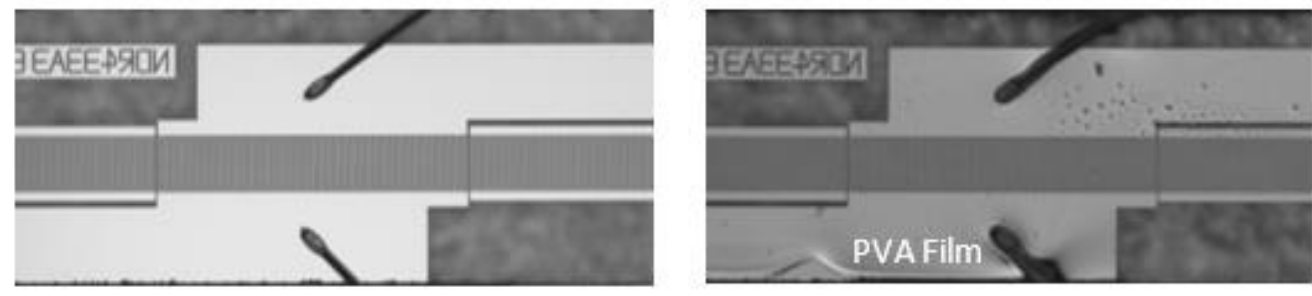

Figure 4.3 The SAW resonator before the PVA film deposition and with the PVA film.

\subsection{4}

\section{PVA Film mechanical properties characterization}

In order to prepare film samples for the PVA film mechanical properties characterization, the first step was to pour aqueous PVA solution onto a glass plate. The plate was then placed inside an oven at $40^{\circ} \mathrm{C}$ for 24 hours to slowly evaporate the solvent and promote the thermal crosslink of the polymer. The thickness of the film seen in figure 4.4 was measured with a digital micrometer after this process and was found to be $0.25 \mathrm{~mm} \pm 0.05 \mathrm{~mm}$. 
Tensile tests were carried out by an INSTRON universal testing machine at room temperature to determine the Young 's modulus of the PVA, according to the ASTM international standard D882-12 - Standard Test Method for Tensile Properties of thin Plastics sheeting [https://www.astm.org/Standards/D882.htm].

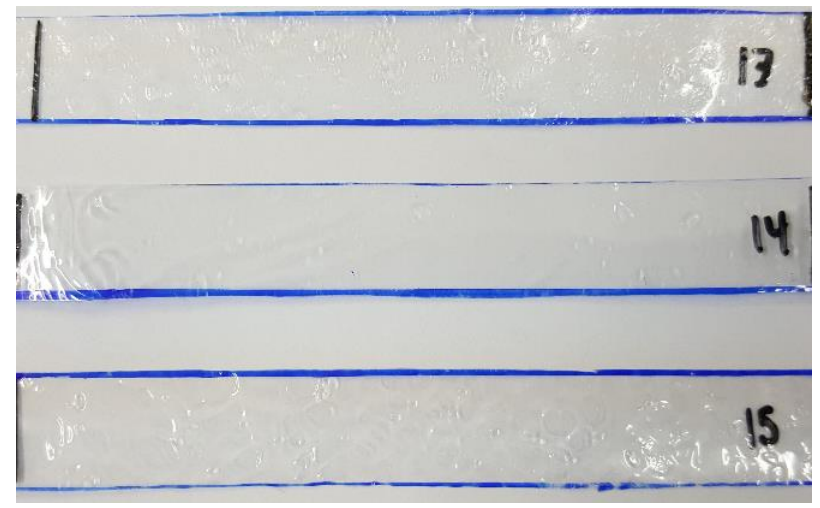

Figure 4.4. PVA film for mechanical test

Around 15 samples with following dimensions $1.5 \times 15 \mathrm{~cm}$ were tested in a stress-strain classical test as shown in figure 4.5. The loads type exerted were by grip separations, consisting of an initial grip to grip distance of $10 \mathrm{~cm}$ and a grip separation rate of $0.1 \mathrm{~cm} / \mathrm{min}$ leading to an initial strain rate of 0.1 , which is in accordance to the ASTM D882-12.

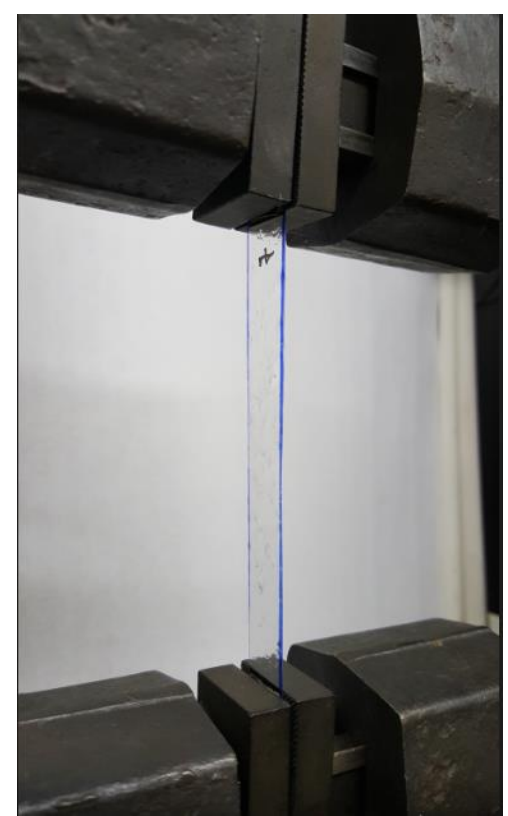

Figure 4.5 PVA films mechanical test

The data registered by the machine and delivered to us consisted of the values for samples deformations and respective stresses exerted. The corresponding relation is represented for each sample, as shown in figure 4.6. 
With the aim to obtain the Young's modulus, calculation of a linear fitting in the initial linear portion of the stress-strain curve, gave the curves slope and corresponding Young's modulus of all the 15 samples. The values of the modulus calculated are summarized in the table 4.2.

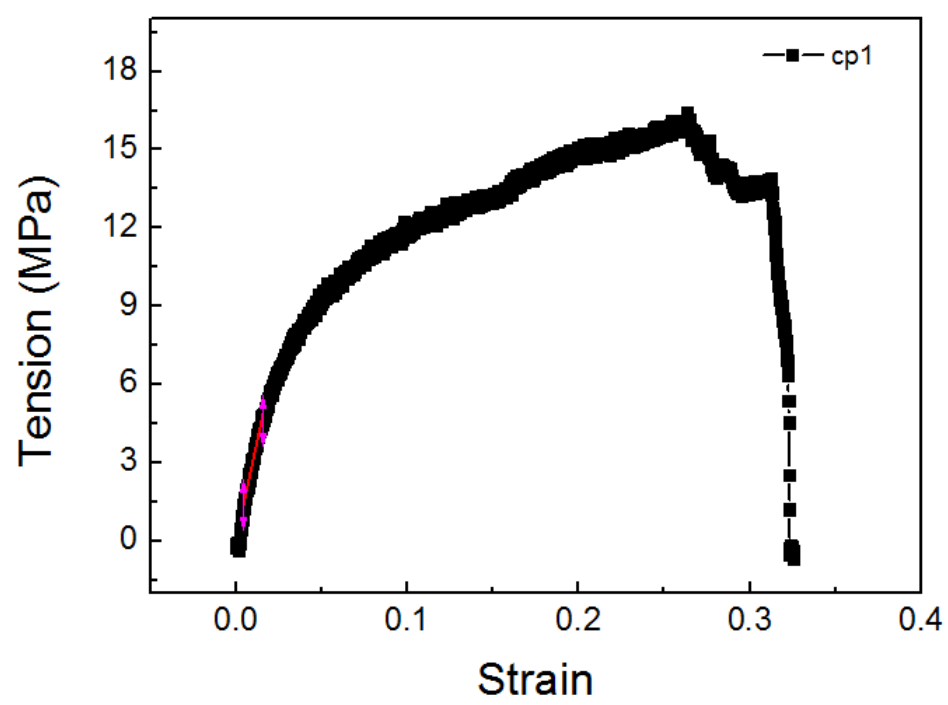

Figure 4.6 Example of a Tension stress- strain curve for sample 1

Due to early fail or break point close to the grip zones, samples like CP 13 , CP 14, CP 15 can't be taken into account for Young determination results.

Table 4.2 Young modulus of the 15 samples in GPA.

\begin{tabular}{|c|c|}
\hline CP & E(GPa) \\
\hline $\mathbf{1}$ & 0.305 \\
\hline $\mathbf{2}$ & 0.262 \\
\hline $\mathbf{3}$ & 0.109 \\
\hline $\mathbf{4}$ & 0.116 \\
\hline $\mathbf{5}$ & 0.101 \\
\hline $\mathbf{6}$ & 0.246 \\
\hline $\mathbf{7}$ & 0.258 \\
\hline $\mathbf{8}$ & 0.441 \\
\hline $\mathbf{9}$ & 0.339 \\
\hline $\mathbf{1 0}$ & 0.201 \\
\hline $\mathbf{1 1}$ & 0.128 \\
\hline $\mathbf{1 2}$ & 0.446 \\
\hline
\end{tabular}


Modulus values shown in the table 4.2 are in the range of $0.1-0.5 \mathrm{GPa}$ in accordance with other studies from several authors [54][55].

\subsection{5}

\section{Measurement system diagram of the for the humidity sensor based on SAW}

As mentioned above, the principle of operation of the humidity sensor is to correlate the difference between the frequencies of the resonators with the surrounding humidity.

SAW devices can be operated wirelessly with energy supply and interrogation studied by several authors [19], [56], [57], but it requires the design of an antenna and an emitter/receiver that was not feasible at the moment, then a wired configuration was used.

Therefore, the topology used for this wired scheme contains an oscillation circuit with a Colpitts oscillator with collector output. This oscillator uses a combination of inductors ( $\mathrm{L}$ ) and capacitors $(\mathrm{C})$ to produce an oscillation at a specific frequency. The distinguishing feature of the Colpitts oscillator is that the feedback for the active device is taken from a voltage divider made of two capacitors in series across the inductor. The oscillating circuits were designed for a commercially avaliable one-port SAW resonator D02 (from HIB) operating at 433.92 MHz with electrical specifications shown in Table

Table 4.3 Electrical specifications of the D02 from HIB.

\begin{tabular}{|c|c|c|c|c|}
\hline Characteristic & Units & Minimum & Typical & Maximum \\
\hline Center frequency & $\mathrm{MHz}$ & 433.845 & 433.92 & 433.995 \\
\hline $\begin{array}{c}\text { Insertion Loss } \\
\begin{array}{c}\text { Unloaded Quality } \\
\text { factor }\end{array}\end{array}$ & $\mathrm{dB}$ & - & 1.2 & 2.0 \\
\hline $\begin{array}{c}\text { Aging of Fc } \\
\text { Motional } \\
\text { capacitance }\end{array}$ & $\mathrm{ppm}$ & & 14500 & \\
\hline Motional inductance & $\mu \mathrm{H}$ & & 3 & $10 /$ year \\
\hline Motional resistance & $\mathrm{Ohm}$ & & 44 & \\
\hline Parallel capacitance & $\mathrm{pF}$ & & 3.2 & \\
\hline $\begin{array}{c}\text { Temperature } \\
\text { coefficient }\end{array}$ & $\mathrm{ppm} / \mathrm{C} * 2$ & & 0.032 & \\
\hline Turnover To & $\mathrm{Deg} . \mathrm{C}$ & 20 & & 50 \\
\hline Package size & & \multicolumn{2}{|l}{} \\
\hline
\end{tabular}


The oscillating circuit was simulated with Genesys Keysight Software. The Figure 4.8.a,b shows the Colpitts-SAW schematic circuit used and the oscillator circuit designed at $433.833 \mathrm{Mhz}$ and $-4 \mathrm{dBm}$. In the simulated model, the SAW resonator was represented by the BVD equivalent circuit (Butterworth-Van Dyke Equivalent Circuit), which consists of an in series resonator LCR circuit in parallel with a capacitance $\mathrm{C}$. The circuit boards were fully developed in the Laboratory using the dry film technique and characterized with a Keysight network analyzer.

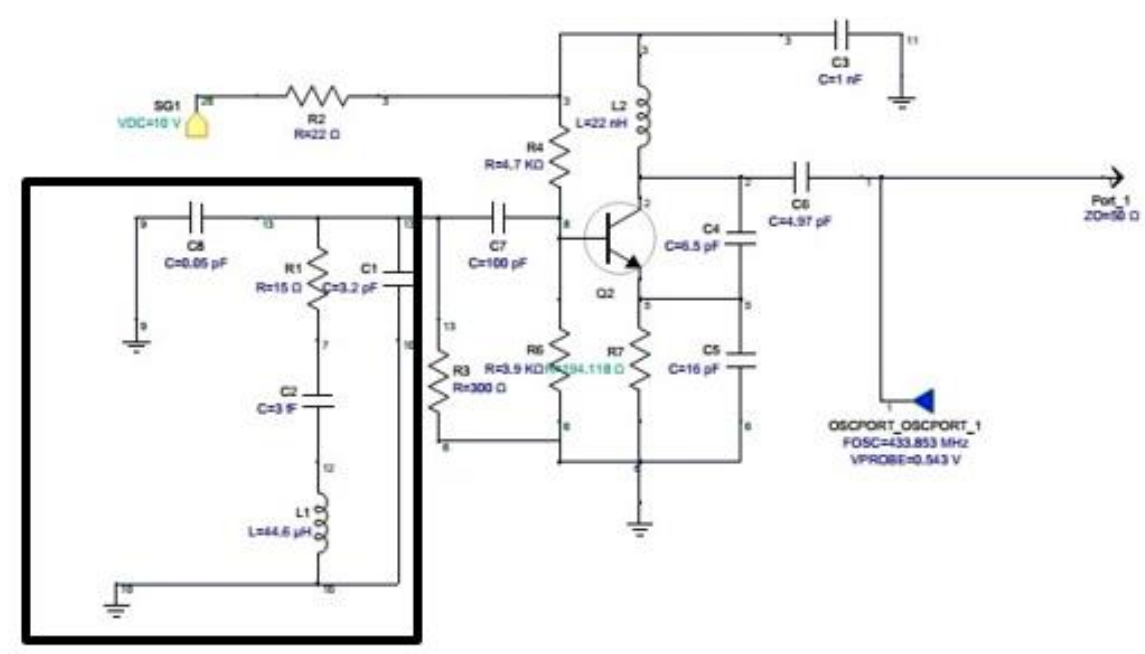

a.
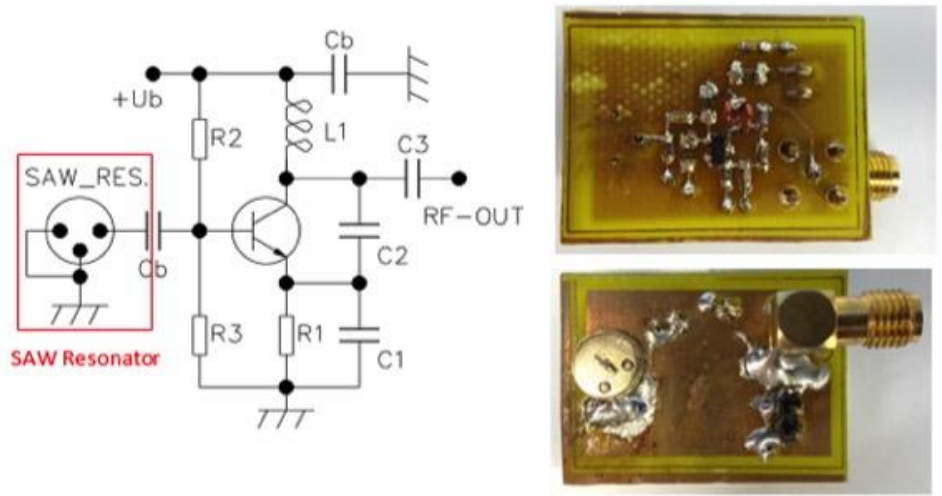

b.

Figure 4.8.a Colpitts schematic circuit. b. Schematic circuit and real oscillator circuit (top and bottom views).

\section{2}

\section{Wired Interrogation System for Saw Sensors}

The frequency-domain measurement method has been adopted in this study. The two resonators were connected to the input ports of a mixer and 
simultaneously connected to a filter, a frequencymeter and a computer, in series configuration, as shown in figure 4.9. The output for this type of setup is a signal corresponding to the difference of frequencies between the reference and the humidity SAW resonators.

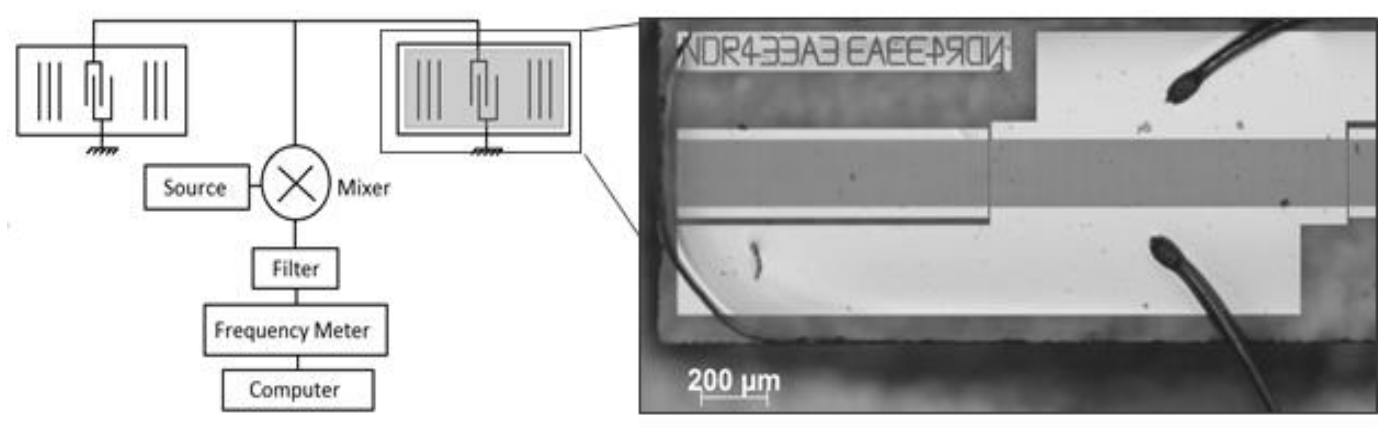

Figure 4.9 Diagram of the measurement system for the SAW humidity sensor. The blowup shows the SAW resonator with the PVA film

One advantage of acquiring the signal by this method is the low cross-talk between the measurand of interest and other variables. Since only one of the resonators is sensitive to humidity, noise originated from frequency variations due to factors other than humidity changes will be reduced. For example, if the temperature increases, both resonators will exhibit the same change in frequency, which will be eliminated after going through the mixer. Another advantage of using the mixer is that the frequency of operation is reduced because SAW sensors operate in the hundreds of megahertz frequency range, whereas the mixer enables working with frequencies in the tens of megahertz range. The mixer thus will allow the use of microcontrollers or digital signal processing circuits to treat the output signal.

\section{3}

\section{Experimental setup}

In order to evaluate the humidity sensor performance, both resonators were placed in a climatic chamber (Votsch VCL 4010) capable of varying the relative humidity from $10 \%$ to $90 \%$. Both resonators were supplied by $10 \mathrm{~V}$ and the power before the mixer was approximately $3 \mathrm{dBm}$. However, the filter output showed only $-4.7 \mathrm{dBm}$, this attenuation is caused by the insertion loss of the mixer. Additionally, a $50 \mathrm{MHz}$ low-pass filter was placed after the mixer output to remove high frequencies. 
Thus, the signal was measured with the frequency meter (model FCA-3103 from Tektronik) operating at frequencies up to $3 \mathrm{GHz}$, with $\mu \mathrm{Hz}$ resolution. This frequency meter allows direct communication with the PC via USB cable. Figure 4.10 shows the experimental setup within the climatic chamber, two resonators connected to the mixer and to the low-pass filter, an external source, the frequency meter and the PC.

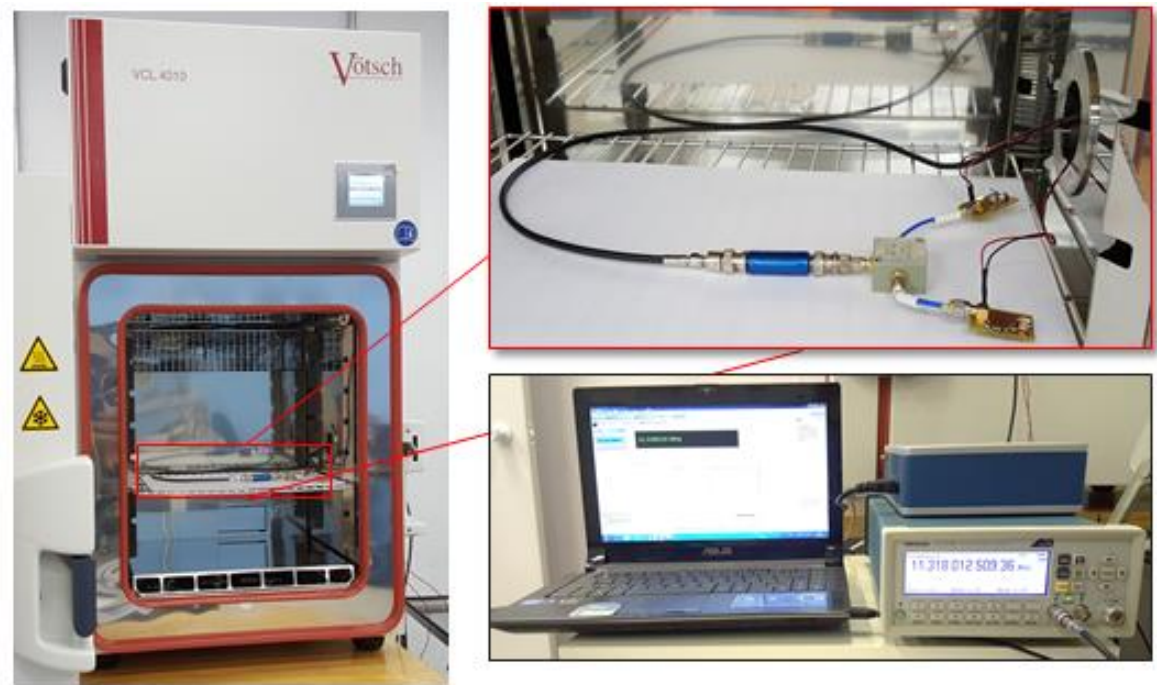

Figure 4.10 Experimental setup for humidity measurements.

\section{4}

\section{Methodology and Results}

In this Section, we discuss the results obtained in five tests performed with the humidity SAW sensor system. The purpose of these tests was to evaluate the performance of the interrogating system and characterize the sensor. The operation parameters for each of the five tests are shown in Table. The quantitative result for these tests is the frequency output of the mixer, filtered by the low-pass filter and each test carried out is described below.

In Test 1, the temperature remained constant while the humidity varied from $60 \%$ to $90 \%$ with steps of $3 \%$. In this case, the objective was to determine the frequency behavior as a function of humidity.

In Test 2, the temperature also remained constant, while the humidity varied from $60 \%$ to $90 \%$ with steps of $10 \%$ for two cycles. In that case, the aim was to determine the response time and the hysteresis of the sensor. 
In Test 3, the same parameters of Test 2 were used, but the test was performed 3 weeks after Test 2 . For Test 3 , the goal was to determine the effects of sensor aging.

Test 4 and test 5 had the purpose of analyzing the frequency behavior as a function of temperature. In Test 4 the humidity was kept constant at $70 \%$, while the temperature varied from $30^{\circ} \mathrm{C}$ to $50^{\circ} \mathrm{C}$ with steps of $5^{\circ} \mathrm{C}$. In Test 5 the same parameters of Test 4 were used, but without the humidity SAW resonator. The idea of this test was to determine the frequency behavior as a function of oscillator circuit temperature, i.e., without the effect of the PVA film.

Table 4.4 Operation parameters of characterization tests

\begin{tabular}{|c|c|c|c|}
\hline TEST & TEMPERATURE & HUMIDITY & OBSERVATION \\
\hline $\mathbf{1}$ & $30^{\circ} \mathrm{C}$ & $\begin{array}{c}60 \%-90 \% ; 3 \% \\
\text { steps }\end{array}$ & \\
\hline $\mathbf{2}$ & $30^{\circ} \mathrm{C}$ & $\begin{array}{c}60 \%-90 \% ; 10 \% \\
\text { steps }\end{array}$ & \\
\hline $\mathbf{3}$ & $30^{\circ} \mathrm{C}$ & $\begin{array}{c}60 \%-90 \% ; 10 \% \\
\text { steps }\end{array}$ & Performed 3 weeks after Test 2 \\
\hline $\mathbf{4}$ & $\begin{array}{c}30^{\circ} \mathrm{C}-50^{\circ} \mathrm{C} ; 5^{\circ} \mathrm{C} \\
\text { steps }\end{array}$ & $70 \%$ & $\begin{array}{c}\text { Humidity SAW resonator } \\
\text { removed }\end{array}$ \\
\hline $\mathbf{5}$ & $\begin{array}{c}30^{\circ} \mathrm{C}-50^{\circ} \mathrm{C} ; 5^{\circ} \mathrm{C} \\
\text { steps }\end{array}$ & $70 \%$ & \multicolumn{2}{c}{} \\
\hline
\end{tabular}

\subsection{1}

\section{Test 1:}

The frequency and humidity as functions of time, as well as the calculated frequency of the sensor as function of humidity, are shown in figure 4.11 for Test 1. Note that, as the humidity increases, the frequency decreases mostly with a non-linear pattern. However, from $80 \%$ to $90 \%$, an almost linear behavior is observed. Different authors have pointed out that the change in mass and conductivity of the sensitive film are two main variables that may induce resonant frequency shift of the SAW sensors [58][59]. 

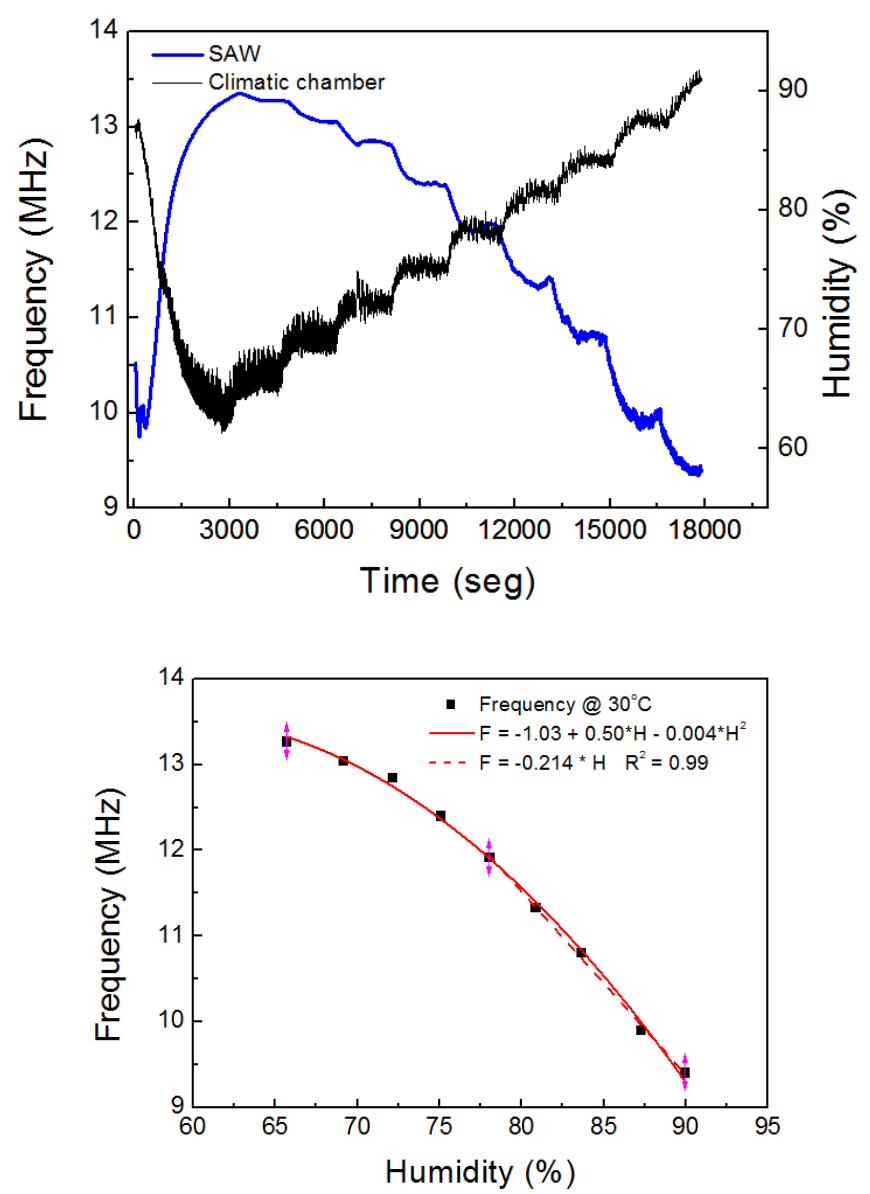

Figure 4.11 Test 1: a. Measured frequency and humidity; $b$. Frequency of the sensor versus humidity, where the points are experimental data and the red line was calculated.

\subsection{2}

\section{Test 2:}

As observed in figure 4.12, Test 2 presented reasonably good repeatability for two consecutive tests. Assuming that the response time has an exponential behavior, it was determined as the required time for the humidity to vary from $80 \%$ to $70 \%$. The exponential growth fitting equation indicated that 220 seconds is approximately the necessary time for the system to achieve stability, indicating a reasonable response compared to the climatic chamber time of 114 seconds. 

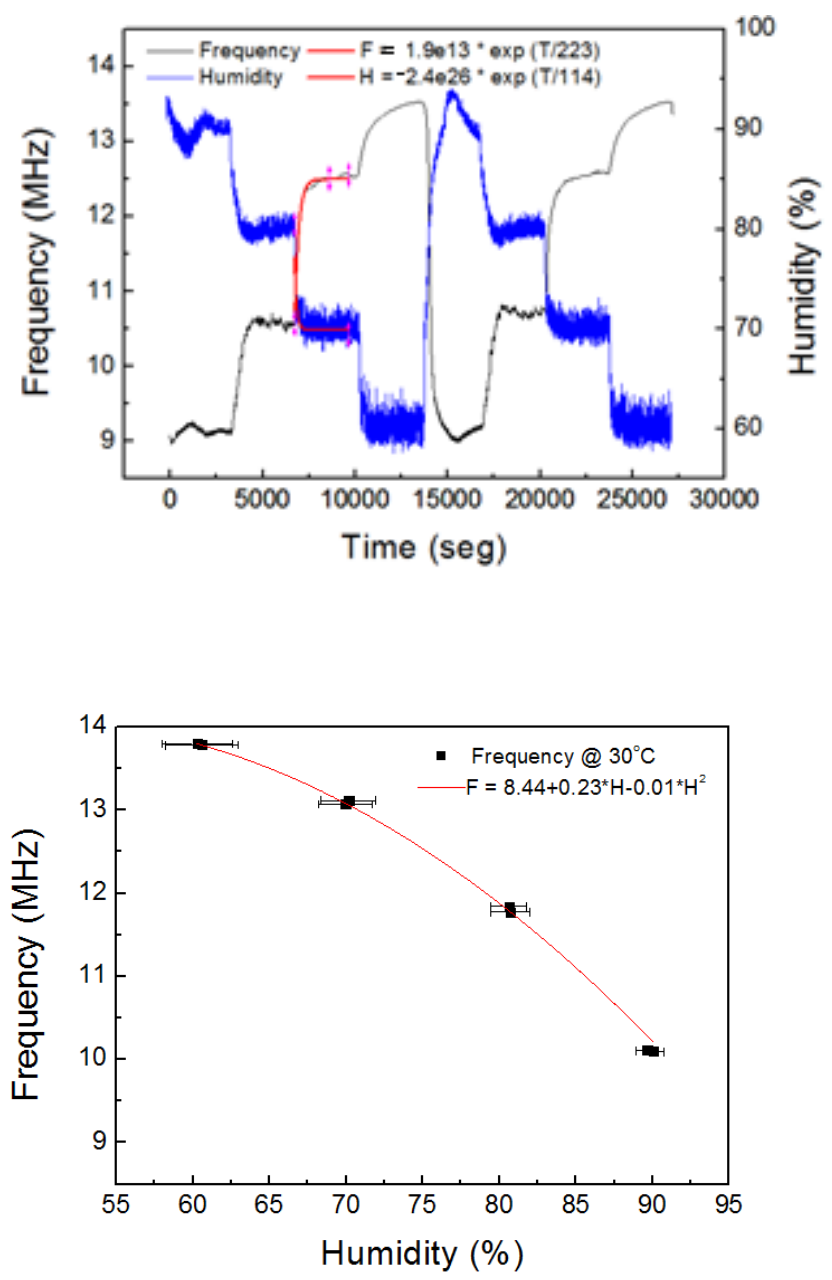

Figure 4.12 Test 2: a. Measured frequency and humidity; $b$. Frequency of the sensor versus humidity, where the points are experimental data and the red line was calculated.

\subsection{3}

Test 3:

The aging of these PVA films is known to be highly dependent on its morphology and on the environment in which it is used. Particularly, Test 3 is a simple preliminary test, in which no standard was used. It is shown that there is a slight difference between Test 2 and Test 3 , as it can be observed in figure 4.13 knowing that Test 3 was performed 3 weeks after Test 2 . In order to identify possible causes for the change in frequencies with time, the SAW surface was observed with the aid of a microscope. As displayed in Figure 4.13, there are some impurities over the surface derived from the metallic case removal process. This may has increased discontinuities on the propagation of the SAW, which could explain the difference in the frequency response observed. Further investigations should be performed in order to confirm these results. 

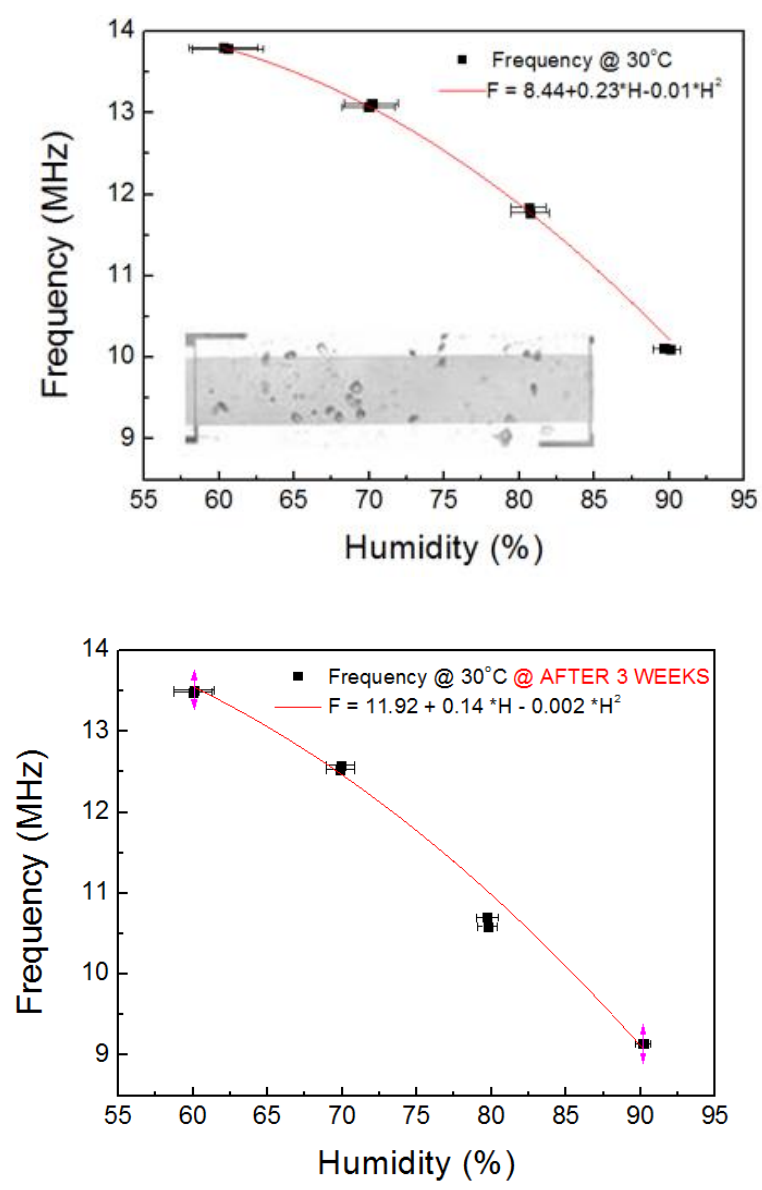

Figure 4.13 Test 3: Comparison of the frequency response of the sensor versus humidity after 3 weeks. The points are experimental data and the red line was calculated.

\subsection{4}

\section{Teste 4 - 5:}

Regarding the frequency behavior as a function of temperature, two significantly different results were observed in Test 4 (with the humidity SAW resonator) and Test 5 (without the humidity SAW resonator). While the system with the humidity SAW resonator shows a linear behavior as a function of temperature, a polynomial behavior is observed when the humidity SAW resonator was taken out of the system. Figure 4.14 shows the corresponding results for Test 4 and Test 5. 

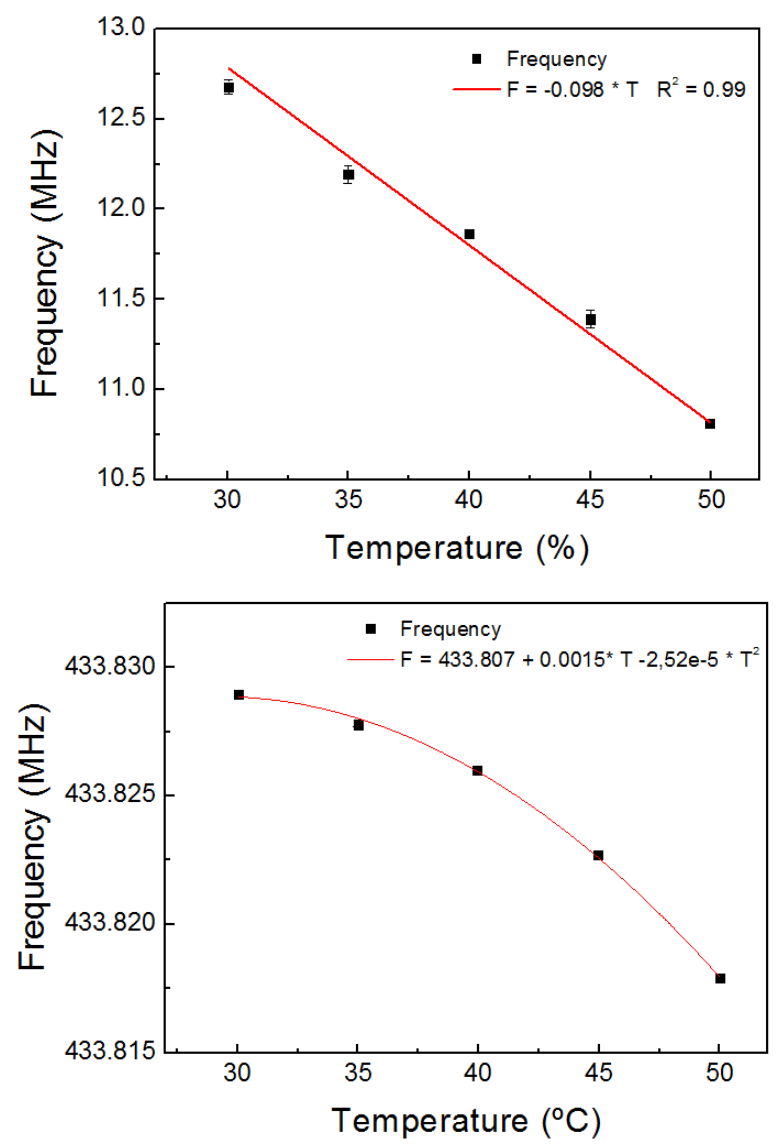

Figure 4.14. Test 4: a. Frequency versus temperature (system with the humidity SAW resonator); b. Frequency versus temperature (system without the humidity SAW resonator).

\section{5}

\section{Analytical SAW Mass-only Response}

From a literature research, SAW gas sensors behavior can be modeled with two classes of analytical expressions. The first one describes the frequency shift due to the sensing layer deposition and the other one treats the gas absorption by this sensing film [18], [45], [60].

In this Section, an analytical model was considered to study the variations in the SAW device operating frequency caused by changes in the PVA film mass.

As presented by Ballantine [15], the general working relationship between the frequency changes and mass loading effect for any acoustic wave device can be expressed based on mass sensitivity as follows:

$$
\Delta f=\operatorname{Sm} * \Delta \mathrm{m}
$$


The term $\Delta \mathrm{m}$ is the variation in mass per unit area in $\left[\mathrm{g} / \mathrm{cm}^{2}\right]$ of the PVA film expressed by its density multiplied by its thickness, as given in equation $4.2 . S_{m}$ is the mass sensitivity term, which is a device-specific constant that depends on the piezoelectric substrate material, device dimensions and the acoustic mode. In the case of our humidity sensor consisted of a ST-quartz SAW resonator, this term is given by the equation 4.3 [15].

$$
\begin{aligned}
& \Delta \mathrm{m}=h * \rho\left[\mathrm{g} / \mathrm{cm}^{2}\right] \\
& \mathrm{Sm}=1.26 * f^{2}\left[\mathrm{~Hz}^{*} \mathrm{~cm}^{2} / \mu \mathrm{g}\right]
\end{aligned}
$$

Where $f$ is the operating frequency of the device.

These relations show that having a thinner polymer sensing layer would cause a reduction of $\Delta \mathrm{m}$, but would improve device sensitivity as less analyte quantity is needed to feel a change.

Regarding the mass loading effect due to the sensing film deposition, the first author Wohltjen in 1984, implemented the Tiersten formula (1978) relating wave velocity change with sensing film properties. This formula was derived from the application of the perturbation method in the wave equation solutions, with the aim to analyze the response of polymer-coated SAW sensors [44]. Hence the frequency shift $\Delta \mathrm{f}_{\mathrm{s}}$ due to a thin non-conductive film deposition is given by:

$$
\Delta f_{s}=\left(k_{1}+k_{2}\right) * F^{2} * h * \rho-k_{2} * F^{2} * h * \frac{4 \mu}{V^{2}} * \frac{\lambda+\mu}{\lambda+2 \mu}
$$

Where $F$ is the centre frequency of a SAW device, $V$ is the wave velocity in the substrate, and $k_{1}, k_{2}$ are substrate material constants, $h$ is the coating thickness, $\rho$ is the coating density, $\mu$ and $\lambda$ are the Lamé constants of the coating material where the first one is also called the shear modulus.

The first term in the equation represents the mass sensitivity and the second term represents the effect of the film stiffness on the device frequency, which could be neglected if the film is a polymer [44] as shown below:

$$
\Delta f_{s}=\left(k_{1}+k_{2}\right) * F^{2} * h * \rho
$$

Several models describing the frequency shift due to gas adsorption have been proposed in literature and the method used in our work is explained in the next section. 


\subsection{1}

\section{Partial density Method:}

This model presented in Sielman et. al. [43] analysed frequency variations in SAW devices caused by the absorption of organic gases into a coating polymer film in a Flexural plate wave (FPW). In this study that phenomena is represented through a shift of the film density, where its expression is given as follows:

$$
\rho(p a r)=\mathrm{k} * \mathrm{c} * \mathrm{M}
$$

In the equation, $\rho$ (partial) is the density variation in the polymer film due to absorption of water vapor, $\mathrm{M}$ is the target gas molar mass, $\mathrm{k}$ is the partition coefficient and $\mathrm{C}$ is the vapor concentration in the gas phase $\frac{\text { moles }}{\mathrm{m}^{3}}$ is computed through the Gas law as follows:

$$
\begin{aligned}
& c=\frac{c_{p p m} * P}{R * T} \\
& \text { And } \\
& \mathrm{c}_{\mathrm{ppm}}=\frac{P_{w}}{P-P_{w}} * 1 e^{6}
\end{aligned}
$$

With $\mathrm{c}_{\mathrm{ppm}}$ being the concentration in parts per million, $P$ is the atmospheric pressure, $R$ is the gas constant, $T$ is the temperature and $P_{w}$ is the partial pressure of water vapor.

This $\mathrm{k}$ coefficient is obtained experimentally, where in applications to gas/polymer interfaces describes a linear ratio of the analyte concentration in the absorbent material (polymer) to the analyte concentration in the vapor phase. This partition coefficient is a measure of the sorption strength as a function of the sorbent material and sensor's operating temperature, some literature values are between 19500 and 20500 [47][61][44] and 7000-12000 by other authors.

This method then uses equations $4.6,4.7$ and 4.8 results to insert them into equation 4.5 to obtain the frequency shift of the SAW. The result is a curve of the analytical relation between humidity variations and frequency shift of the SAW humidity sensor. In the figure 4.18 this analytical result together with the experimental test are shown with the aim to be compared and analyzed their behavior. 


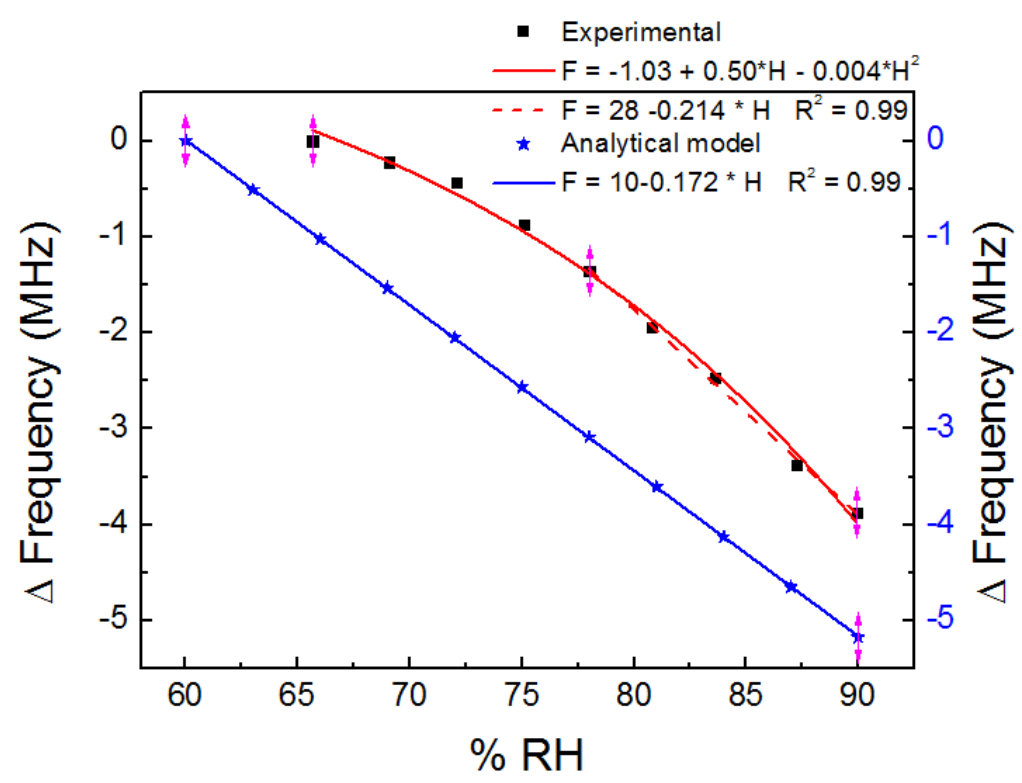

Figure 4.18 Frequency shift results using partial density method compared to Experimental SAW Humidity sensor

Regarding this method, as could be seen in the above figure, the trend of the analytical curve coincide with our experimental result. The increase in the negative value of the figure means a decrease in the operating frequency respect to the initial frequency of the SAW coated, in according with the negative increase difference of the experimental curve. In spite of the exact values does not agree at all, the slope of the curves have similar values, $-0.206 \mathrm{MHz} / \% \mathrm{RH}$ for the experimental and $-0.172 \mathrm{MHz} / \% \mathrm{RH}$ for the analytical model. 


\section{5 \\ Finite Element model for SAW devices}

This section is focused on the finite element modeling of the humidity sensor based on SAW resonator. During the course of this research, a 2D FEM model was implemented with the software package COMSOL Multiphysics $\AA^{\circledR}$ version 5.2 using the structural mechanics and piezoelectric modules. The simulations only takes into account the mass change due to humidity absorption of PVA film. Finally, these simulation results will be compared to experimental results.

\section{1}

\section{Finite Element Analysis (FEA) for SAW Devices}

The type of analysis studied in literature for SAW devices consists of two parts: a modal analysis and a harmonic analysis. Each part is described below.

Modal analysis: in this analysis, the homogeneous solution obtained corresponds to the eigenmodes of the SAW problem. This analysis gives the frequency at which a particular mode resonates for a given wavelength, and as there is no propagation into the media, two frequencies are obtained corresponding to the SAW modes. These two frequencies are the edges of a stopband representing resonance and anti-resonance.

Harmonic analysis: by application of harmonic voltage around the modal frequencies, the particular solution corresponding to the excited electrical and mechanical fields is found. The frequencies, the absolute displacements and electric field distributions determined from the previous analysis are used here to calculate the admittance of the device. This device admittance is used to analyze the frequency and electrical response of the PVA coated SAW resonator due to the humidity variation.

\subsection{1}

\section{Survey of important characteristics of the SAW and coating film}

Information on geometry and material properties are the necessary inputs of the numerical model. Thus, a commercial SAW with a PVA coating film was inspected for retrieving the desired information. 
The substrate used in the simulation is ST-X cut quartz, their material constants have been reported by Zhao [45] having the corresponding Euler angles $(0,132.75,0)$ with the following material constants:

The dielectric matrix:

$$
\varepsilon=\left[\begin{array}{ccc}
0.3921 & 0 & 0 \\
0 & 0.4005 & 0.0091 \\
0 & 0.0091 & 0.4019
\end{array}\right]^{x 10^{-10}}
$$

The piezoelectric coefficients:

$$
e=\left[\begin{array}{cccccc}
0.171 & -0.1327 & -0.0383 & 0.0821 & 0 & 0 \\
0 & 0 & 0 & 0 & 0.10716 & -0.0720 \\
0 & 0 & 0 & 0 & -0.0990 & 0.0665
\end{array}\right]^{C / m^{2}}
$$

Therefore, the elastic stiffness coefficients matrix of ST-cut quartz is shown below.

$$
c=\left[\begin{array}{cccccc}
86.74 & -8.60 & 27.50 & 1.05 & 0 & 0 \\
-8.60 & 130.74 & -4.81 & -1.84 & 0 & 0 \\
27.50 & -4,81 & 96.63 & 13.44 & 0 & 0 \\
1.05 & -1.84 & 13.44 & 1.22 & 0 & 0 \\
0 & 0 & 0 & 0 & 67.47 & 7.60 \\
0 & 0 & 0 & 0 & 7.60 & 30.35
\end{array}\right]^{x 10^{9} \mathrm{~N} / \mathrm{m}^{2}}
$$

The detailed geometry was determined from the microscopy image shown in figure 5.1. The measured finger width is $2 \mu \mathrm{m}$, and the space between fingers is $1.5 \mu \mathrm{m}$.

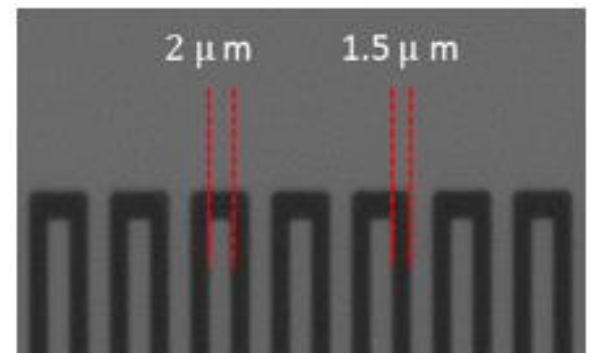

Figure 5.1 Photo of SAW dimensions

The properties of the PVA coating film deposited in the SAW were taken from a literature review [43] and are shown in table 5.2. It is important to notice that the maximum Young's modulus value measured experimentally (section 4.1.4) was just $0.5 \mathrm{GPa}$, which is one order of magnitude lower than the used in the simulation. 
Table 5.2 PVA material properties

\begin{tabular}{|c|c|}
\hline Material & PVA Film \\
\hline Density & $1200 \mathrm{~kg} / \mathrm{m} 3$ \\
\hline Poisson ratio & 0.4 \\
\hline Young modulus & $5 \mathrm{GPa}$ \\
\hline Partition coefficient & 22000 \\
\hline Thickness & $1 \mathrm{um}$ \\
\hline
\end{tabular}

\section{1 .2}

\section{FEM model}

Since there is no variation of amplitudes in the $y$-direction [23], a 2D simplified model was implemented for simulating the SAW behavior. Figure 5.2 shows the schematic drawing of this numerical model. It consists of a periodic unit cell with width $\lambda$ of $7 \mu \mathrm{m}$ and with IDT period $\mathrm{p}$ of $3.5 \mu \mathrm{m}$ with no resonator structures.

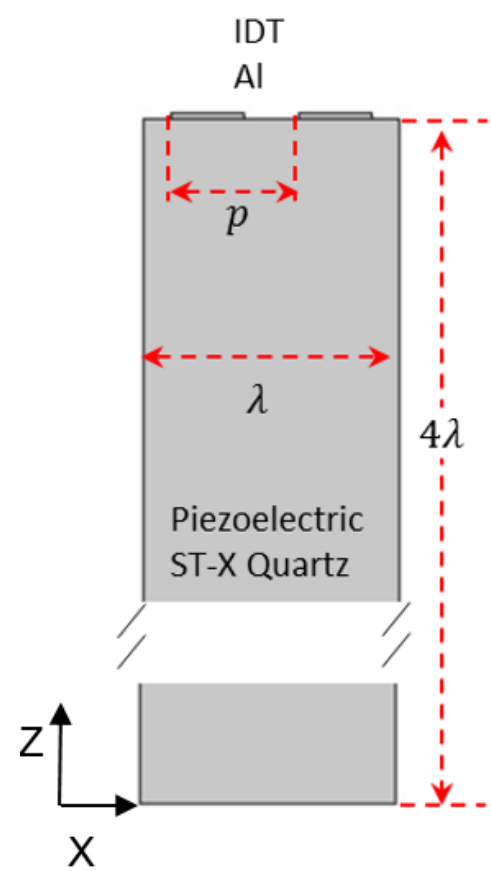

Figure 5.2 Model dimensions used in the simulations.

Considering that $90 \%$ of the acoustic wave energy is confined into one wavelength measured from the surface, only 3-10 wavelengths of the substrate depth are needed to solve the calculations with good accuracy. Thus, in this simulation only four wavelengths were attributed to the substrate depth. In addition, a domain representing the PVA layer is included over the top of the model. 
The boundary conditions (BCs) applied to the model are resumed in Table 5.3. There are two groups of BCs: the first is formed by the mechanical BCs, and the second is formed by the electrical BCs. In addition, the periodic condition is included by applying the periodic continuity boundary condition (PCBC) to the left and right boundaries of the fundamental cell, leading to an infinite transducer cell, as can be seen in Figure 5.3.

Table 5.3 Boundary conditions of the simulation model

\begin{tabular}{|c|c|c|}
\hline Boundary & Mechanical & Electrical \\
\hline$\Gamma_{T}$ & & Zero charge \\
\hline$\Gamma_{B}$ & Fixed & Ground \\
\hline$\Gamma_{L}, \Gamma_{R}$ & \multicolumn{2}{|c|}{ P.C.B.C } \\
\hline
\end{tabular}

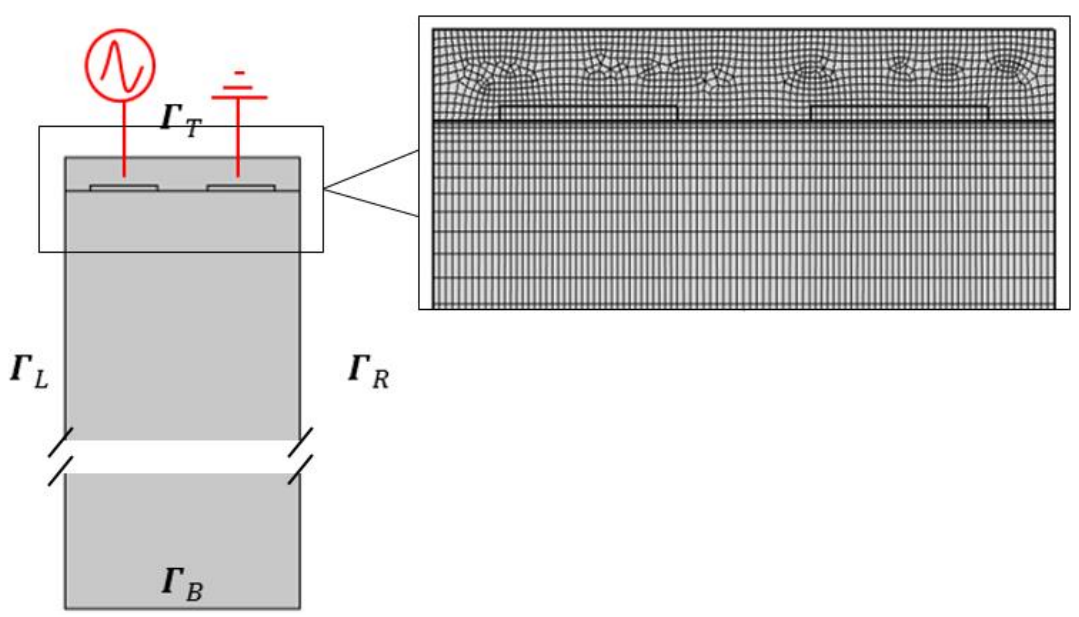

Figure 5.3 Mesh and boundary conditions applied

A mapped mesh was adopted to subdivide the piezoelectric domain, and for the polymer domain, a free quadrilateral mesh was used. A convergence analysis was conducted to make sure the model returned reliable results.

\subsection{3}

\section{Simulation Results}

From the modal analysis, the eigenmodes corresponding to surface acoustic waves were identified. In this case, loads were not applied, and a pair of SAW eigenfrequencies representing the symmetric and antisymmetric mode response, 
are represented in the Figure 5.4. The color corresponds to the displacement amplitude in the $Z$ direction, and the distorted shape is proportional to the actual displacements.

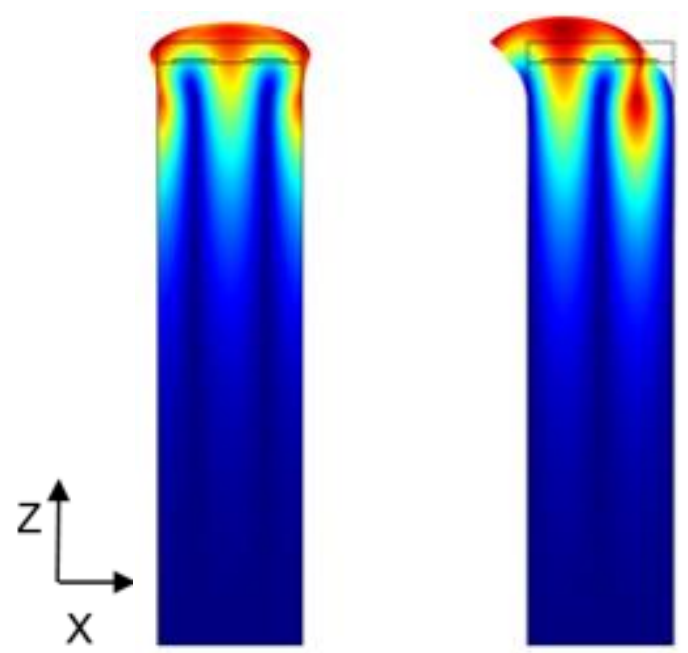

Figure 5.4 Symmetric and antisymmetric SAW modes

In the simulation, a frequency sweep passing through the stopband while applying a drive voltage was performed. The harmonic admittance can be determined from the complete charge distribution of the electrodes $Q$, representing the SAW electrical behaviour using the following relation:

$$
Y=\frac{j \omega Q}{V}
$$

where, $Y$ is the complex admittance, $j$ the imaginary number, $\omega$ the angular frequency and $V$ the drive voltage applied. The figure shows the absoluted value of admittance as a function of frequency. It is within the investigated range of $410 \mathrm{MHz}$ to $460 \mathrm{MHz}$, the highest admittance peak occurs at $429 \mathrm{MHz}$. 


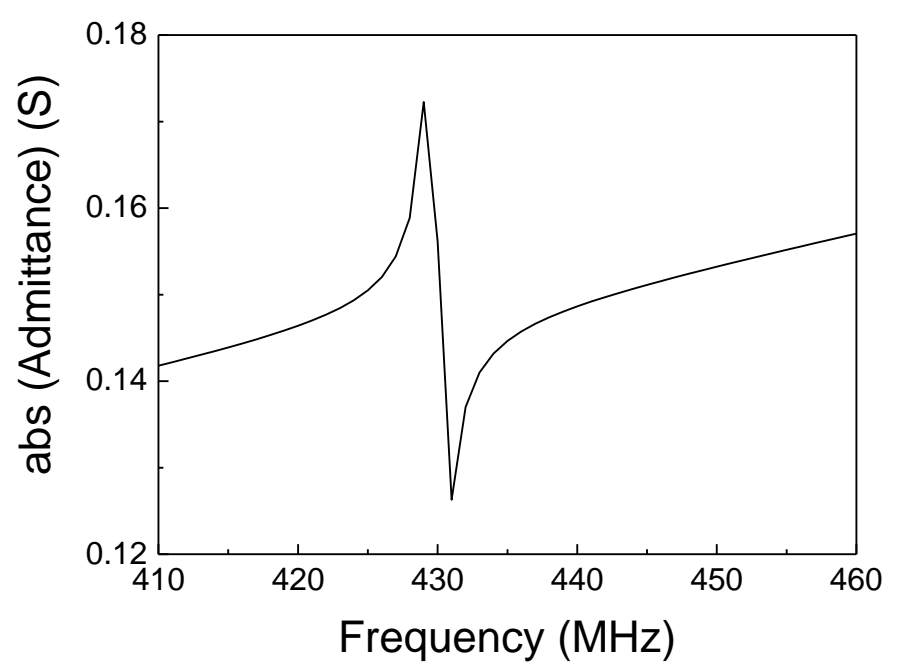

Figure 5.5 SAW admittance

The numerical model evaluted the ressonance frequency change as a function to the mass change of PVA film. The results shown in figure 5.6, revealed a linear decreasing frequency response for the relative humidity increase, in contrast to the experimental results that showed a non linear behavior. However, in this experimental results it is possible to observe an almost linear behavior in the range from $80 \%$ to $90 \%$ humidity. The figure presents a comparison of the resonance frequency between the numerical and experimental results. In both curves, can be observed that within the range from $80 \%$ to $90 \%$ there is small diffrences between their sensitivities (represented by the curves slopes with values of -0.20 for the experiemetal result and 0.183 for the simulated result).

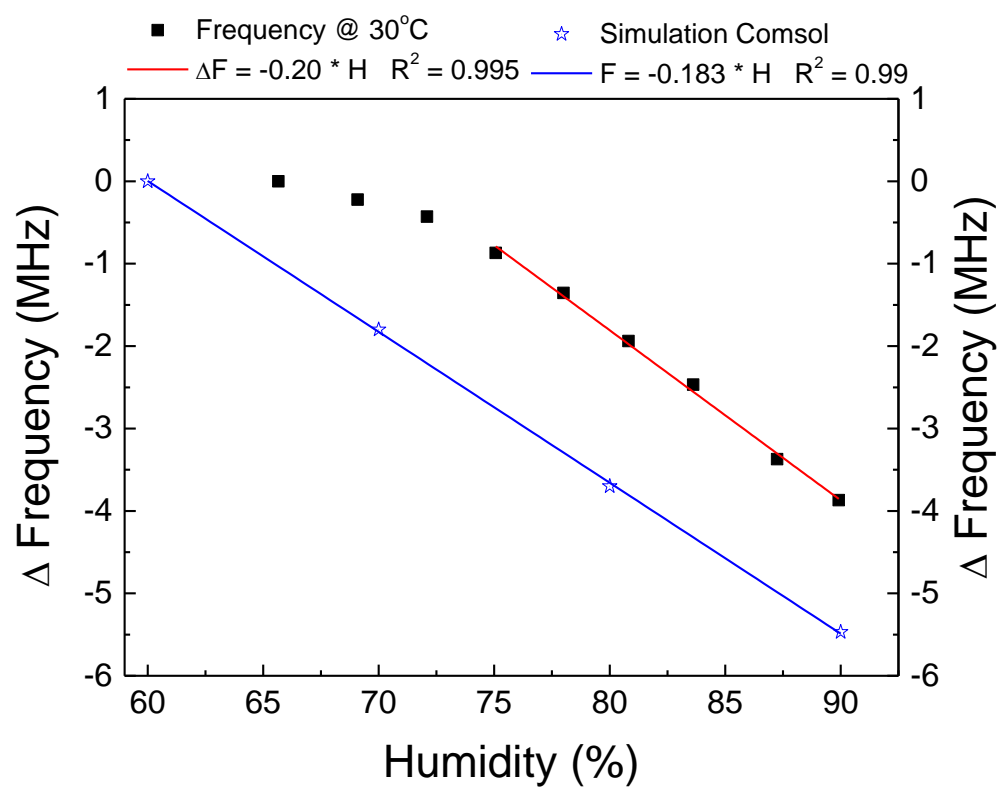


Figure 5.6 Numerical and experimental results comparisons

Nevertheless, It is important to point out that these differences are about $10 \%$ between results, what indicates the need of more extensive studies on the impacts of the PVA Young's modulus and thickness changes. 


\section{6 Conclusions and Future Work}

Regarding the performance of the SAW resonator coated with a PVA film, the experimental results demonstrate the functionality of this approach. These experimental results showed a non linear behavior of the frequency with humidity variations. However, in the curve it is possible to observe an almost linear behavior in the range from $80 \%$ to $90 \%$ humidity with a slope of of $0.20 \mathrm{MHz} /{ }^{\circ} \mathrm{C}$ (which is the sensitivity of the device).

Another series of test regarding temperature variations were carried out, with the aim to evaluate the influence in the sensor performance and circuitry. The linear behavior in the whole system gives a sensitivity of $0,098 \mathrm{MHz} /{ }^{\circ} \mathrm{C}$, while without the humidity sensor a nonlinear behavior was detected. Finally, a preliminary test evaluating the aging effect of the PVA film ( 3 weeks old) shows a reduction in the performance of the sensor as decrease in the operating frequency occurs. Although more test should be carried out to investigate better this phenomenon because there is not much information about it.

In addition, the comparison of these experimental results with the results of the analytical model, showed a slight difference in the sensitivity obtained through the fitting slope for each result curves. Such a difference is about $14 \%$ between the values, what says that an improvement to the model is necessary, noticing that effects on the Young's modulus and the thickness variations were not taken into account.

Finally, the results of the humidity SAW sensor behavior evaluated through numerical simulations, revealed good accordance when compare with the experimental results. Specifically, the sensitivity to humidity variations of the SAW frequency shows a difference of only $8.5 \%$ respected to the values of the slope for each result curve (experimental and analytical). It is worth to point out that the Young's modulus used in the simulation was one order above to that of the experimental result, but still valid following literature values.

For future works, improvements can be made to the numerical and analytical model, and also to the experimental set up. Regarding the model used for analytical and numerical analysis, more effects caused by humidity absorption should be taken into account. As only PVA density variation was selected to be the 
only variable affected with the humidity absorption. These factors could be the PVA thickness and Young's modulus, which were reported by others authors to have their behavior affected.

In addition, an experimental study on the influence of the PVA thickness over SAW response should be carried out to select the optimum size to deposit without affect sensor performance. 


\section{Bibliography}

[1] R. Azevedo and M. B. J. Wijesundara, Silicon Carbide Microsystems for Harsh Environments, vol. 1. Springer, 2011.

[2] A. A. S. Mohammed, W. A. Moussa, and E. Lou, "Mechanical Strain Measurements Using Semiconductor Piezoresistive Material," vol. 5, no. I, pp. 5-6, 2006.

[3] N. Maluf and K. R. Williams, An Introduction to Microelectromechanical Systems Engineering, vol. 13. 2004.

[4] C. Hu, "Chapter 3: Device Fabrication Technology," Mod. Semicond. Devices Integr. Circuit, pp. 59-88, 2009.

[5] P. C. T. Nguyen, "EE 245 : Introduction to MEMS Lecture $8 \mathrm{~m} 2$ : Surface Micromachining Lecture Outline EE C245 - ME C218 Introduction to MEMS Design Fall 2010 Polysilicon Surface-Micromachining Copyright @ 2010 Regents of the University of California Lecture 8m2: Surface Mi," Electr. Eng., pp. 20-22, 2010.

[6] S. Kon, K. R. Oldham, and R. Horowitz, "Piezoresistive and piezoelectric MEMS strain sensors for vibration detection," SPIE_International Soc. Opt. Eng., vol. 6529, p. 65292V-65292V-11, 2007.

[7] J. Rausch, P. Heinickel, B. Koegel, K. Zogal, and P. Meissner, "Experimental comparison of piezoresistive MEMS and fiber bragg grating strain sensors," 2009 IEEE Sensors, pp. 1329-1333, 2009.

[8] R. G. A. R. G. Azevedo, D. G. J. D. G. Jones, A. V. J. A. V. Jog, B. J. B. Jamshidi, D. R. M. D. R. Myers, L. C. L. Chen, X. F. X. Fu, M. M. M. Mehregany, M. B. J. W. M. B. J. Wijesundara, and A. P. P. A. P. Pisano, "A SiC MEMS Resonant Strain Sensor for Harsh Environment Applications," IEEE Sens. J., vol. 7, no. 4, pp. 568-576, 2007.

[9] a a S. Mohammed, W. a Moussa, and E. Lou, "High sensitivity MEMS strain sensor: Design and simulation," Sensors, vol. 8, no. 4, pp. 2642-2661, 2008.

[10] A. Ahmed, S. Mohammed, A. Ahmed, and S. Mohammed, "Utilization of Semiconductors Piezoresistive Properties in Mechanical Strain by Dedication To my parents , my grandmother , and my family ... the persons 
who sacrificed," 2013.

[11] K. E. Wojciechowski, B. E. Baser, and A. P. Pisano, "A MEMS resonant strain sensor operated in air," 17th IEEE Int. Conf. Micro Electro Mech. Syst. Maastricht MEMS 2004 Tech. Dig. January 25, 2004 - January 29, 2004, pp. 841-845, 2004.

[12] J. Fraden, Handbook of Modern Sensors: Physics, Designs, and Applications, 2nd ed. 2010.

[13] Z. Chen and C. Lu, "Humidity Sensors: A Review of Materials and Mechanisms," Sens. Lett., vol. 3, no. 4, pp. 274-295, 2005.

[14] H. Farahani, R. Wagiran, and M. N. Hamidon, Humidity sensors principle, mechanism, and fabrication technologies: A comprehensive review, vol. 14, no. 5. 2014.

[15] D. S. Ballantine, S. J. Martin, and A. J. Ricco, Acoustic Wave Sensors. .

[16] L. Rayleigh, "On Waves Propagated along the Plane Surface of an Elastic Solid," Proc. London Math. Soc., vol. s1-17, no. 1, pp. 4-11, Nov. 1885.

[17] C. Lin, C. R. Lin, S. Yu, G. Liu, C. Hung, and H. Lin, "Study on Wireless Torque Measurement Using SAW Sensors," Appl. Meas. Syst., pp. 109136, 2012.

[18] I. Avramov, "Polymer coated rayleigh SAW and STW resonators for gas sensor applications," Acoust. Waves_From Microdevices to ..., 2011.

[19] D. W. Greve, T.-L. Chin, P. Zheng, P. Ohodnicki, J. Baltrus, and I. J. Oppenheim, "Surface acoustic wave devices for harsh environment wireless sensing.," Sensors (Basel)., vol. 13, pp. 6910-35, 2013.

[20] R. M. White and F. W. Voltmer, "DIRECT PIEZOELECTRIC COUPLING TO SURfACE ELASTIC WAVES," Appl. Phys. Lett., vol. 7, no. 12, pp. 314316, Dec. 1965.

[21] B. Li, H. Al Rowais, and J. Kosel, "Surface Acoustic Wave Based Magnetic Sensors," Model. Meas. Methods Acoust. Waves Acoust. Microdevices, 2013.

[22] N. A. Ramli and A. N. Nordin, "Design and modeling of MEMS SAW resonator on Lithium Niobate," 2011 4th Int. Conf. Mechatronics Integr. Eng. Ind. Soc. Dev. ICOM'11 - Conf. Proc., no. May, pp. 17-19, 2011.

[23] T. Kannan, "Finite Element Analysis of Surface Acoustic Wave Resonators," Organization, no. June, 2006.

[24] S. Härmä, Surface Acoustic Wave RFID Tags: Ideas, Developments, and Experiments, vol. Doctoral, no. February. 2009.

[25] I. J. Oppenheim, N. S. Carey, T.-L. Chin, P. Zheng, and D. W. Greve, 
"Temperature and stiffness correction of SAW devices for wireless strain sensing," Aerospace, vol. 7981, p. 79811F-79811F-8, 2011.

[26] T. Li, H. Hu, G. Xu, K. Zhu, and L. Fang, "Pressure and Temperature Microsensor Based on Surface Acoustic Wave in TPMS," no. September, 2010.

[27] G. a. Borrero, J. P. Bravo, S. F. Mora, S. Velásquez, and F. E. SeguraQuijano, "Design and fabrication of SAW pressure, temperature and impedance sensors using novel multiphysics simulation models," Sensors Actuators A Phys., vol. 203, pp. 204-214, 2013.

[28] F. Della Lucia, P. Zambrozi, F. Frazatto, M. Piazzetta, and a. Gobbi, "Design, fabrication and characterization of SAW pressure sensors for offshore oil and gas exploration," Sensors Actuators A Phys., vol. 222, pp. 322-328, 2015.

[29] "Modelling of Hysteresis and Creep in SAW Strain Sensors," pp. 0-3.

[30] V. Kalinin, G. Bown, and A. Leigh, "P1K-3 Contactless Torque and Temperature Sensor Based on SAW Resonators," Ultrason. Symp. 2006. IEEE, pp. 1490-1493, 2006.

[31] V. Kalinin, "Wireless physical SAW sensors for automotive applications," 2011 IEEE Int. Ultrason. Symp., pp. 212-221, 2011.

[32] M. Penza and G. Cassano, "Relative humidity sensing by PVA-coated dual resonator SAW oscillator," Sensors Actuators, B Chem., vol. 68, no. 1, pp. 300-306, 2000.

[33] K. Hashimoto, Surface Acoustic Wave Devices in Telecommunications Modelling and Simulation. 2000.

[34] A. Auld, Acoustic fields and waves in solids. .

[35] D. Morgan, Surface acoustic wave filters with applications to electronics communications and signal processing. 2010.

[36] C. C. W. Ruppel and T. A. Fieldly, Advances in Surface Acoustic Wave Technology, Systems and Applications (Vol. II). 2001.

[37] E. Royer, Daniel and Dieulesaint, Elastic Waves in Solids I Free and Guided Propagation. 1996.

[38] V. Avramescu, C. Bostan, B. Serban, I. Georgescu, S. Costea, N. Varachiu, and C. Cobianu, "Surface Acoustic Wave Devices and Their Sensing Capabilities," pp. 27-36, 1885.

[39] C. Campbell, Surface acoustic wave devices and their signal processing applications. 1989.

[40] S. A. Wave, L. Acoustic, W. Hydrogen, and G. Sensors, "S ensitivity 
Comparison between Surface Acoustic Wave and Lamb Acoustic Wave Hydrogen Gas Sensors," no. August, pp. 1-10, 2013.

[41] M. J. Vellekoop, "Acoustic wave sensors and their technology," Ultrason., vol. 36, no. 1-5, pp. 7-14, 1998.

[42] A. A. Vives, Piezoelectric transducers and applications. 2008.

[43] C. Sielmann, "Design and Performance of All-Polymer Acoustic Sensors," 2012.

[44] J. W. Grate, A. Snow, D. S. Ballantine, H. Wohltjen, M. H. Abraham, R. A. McGill, and P. Sasson, "Determination of partition coefficients from surface acoustic wave vapor sensor responses and correlation with gas-liquid chromatographic partition coefficients," Anal. Chem., vol. 60, no. 9, pp. 869875, 1988.

[45] Y. G. Zhao, M. Liu, D. M. Li, J. J. Li, and J. Bin Niu, "FEM modeling of SAW organic vapor sensors," Sensors Actuators, A Phys., vol. 154, no. 1, pp. 3034, 2009.

[46] E. T. Zellers, S. a Batterman, M. Han, and S. J. Patrash, "Optimal coating selection for the analysis of organic vapor mixtures with polymer-coated surface acoustic wave sensor arrays.," Anal. Chem., vol. 67, no. 6, pp. 1092-106, 1995.

[47] J. W. Grate and M. Klusty, "Surface acoustic wave vapor sensors based on resonator devices," Anal. Chem., vol. 63, no. 17, pp. 1719-1727, 1991.

[48] M. Hribšek and D. Tošić, "Analysis and Modelling of Surface Acoustic Wave Chemical Vapour Sensors," Cdn.Intechopen.Com, no. September, 2010.

[49] L. Wang, J. Liu, and S. He, "The development of love wave-based humidity sensors incorporating multiple layers," Sensors (Switzerland), vol. 15, no. 4, pp. 8615-8623, 2015.

[50] J. Li, J. Suo, and R. Deng, "Structure, mechanical, and swelling behaviors of poly (vinyl alcohol)/SiO2 hybrid membranes," J. Reinf. Plast. Compos., vol. 29, no. 4, pp. 618-629, 2010.

[51] J. Krzeminski and H. Molisak-tolwinska, "Journal of Macromolecular Science: Part A - Chemistry The Structure of Water-Swollen Poly ( Vinyl Alcohol ) and the Swelling Mechanism," no. June 2013, pp. 37-41.

[52] N. A. Kadri, M. G. Raha, and B. Pingguan-Murphy, "Polyvinyl alcohol as a viable membrane in artificial tissue design and development.," Clinics (Sao Paulo)., vol. 66, no. 8, pp. 1489-1494, 2011.

[53] V. M. and Konidari, K. G. an. Papadokostaki, and M. Sanopoulou, "MoistureInduced Effects on the Tensile Mechanical Properties and Glass-Transition 
Temperature of Poly(vinyl alcohol) Films," J. Appl. Polym. Sci., vol. 120, no. 7, pp. 449-456, 2011.

[54] T. Peijs, "Mechanical properties of poly(vinyl alcohol) fiber and composite," Composites, vol. 26. pp. 83-90, 1995.

[55] T. Fukumori and T. Nakaoki, "Significant Improvement of Mechanical Properties for Polyvinyl Alcohol Film Prepared from Freeze/Thaw Cycled Gel.," Open J. Org. Polym. Mater., vol. 3, no. 110-116, pp. 110-116, 2013.

[56] W. C. Wilson, D. C. Malocha, N. Kozlovski, D. R. Gallagher, B. Fisher, J. Pavlina, N. Saldanha, D. Puccio, and G. M. Atkinson, "Orthogonal Frequency Coded SAW Sensors for Aerospace SHM Applications," IEEE Sens. J., vol. 9, no. 11, pp. 1546-1556, 2009.

[57] V. Kalinin, "Passive wireless strain and temperature sensors based on SAW devices," Radio Wirel. Conf. 2004 IEEE, pp. 187-190, 2004.

[58] L. Fan, S. Zhang, H. Ge, and H. Zhang, "Theoretical optimizations of acoustic wave gas sensors with high conductivity sensitivities," 2012.

[59] M. Hoummady and A. Campitelli, "Acoustic wave sensors: design, sensing mechanisms and applications," Smart Mater. Struct., vol. 6, no. 6, pp. 647657, Dec. 1997.

[60] C. Multiphysics, "SAW Gas Sensor," vol. 3, pp. 1-16, 2013.

[61] C. Sielmann, J. Berring, K. Walus, and B. Stoeber, "Application of an allpolymer flexural plate wave sensor to polymer/solvent material characterization," Proc. IEEE Sensors, no. 2, pp. 3-6, 2012. 
A

SAW data sheet

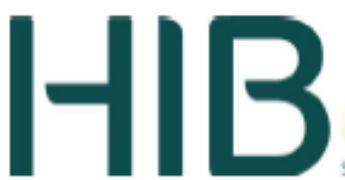

SEMICONDUTORES

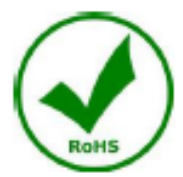

SAW Resonator D02

\section{0 to $980 \mathrm{MHz}$}

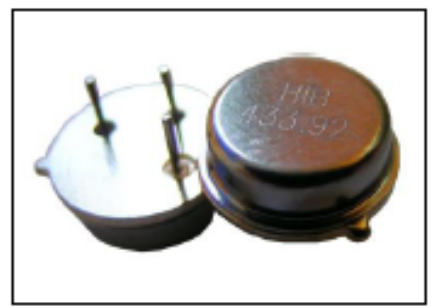

It is a true one-port SAW

FEATURES

resonator in a low profile TO39

- One-port resonator

case. This product is designed

- Fundamental Mode

for remote control and wireless

- Quartz Stabilization

security transmitters.

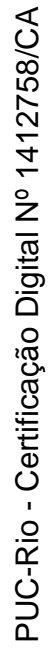

Electrical Specifications

\begin{tabular}{|c|c|c|c|c|c|c|}
\hline Parameter & Symbol & Condition & Min & Typ & Max & Units \\
\hline Frequency Range & $F_{0}$ & & 292.0 & & 980.0 & $\mathrm{MHz}$ \\
\hline Frequency Tolerance & $\Delta \mathrm{F} / \mathrm{F}_{0}$ & at $25^{\circ} \mathrm{C}$ & \pm 50 & & \pm 500 & $\mathrm{KHz}$ \\
\hline Temperature Stability & $T_{c}$ & Ref to $25^{\circ} \mathrm{C}$ & & -0.032 & & $\operatorname{PPM} /\left(\Delta^{\circ} \mathrm{C}\right)^{2}$ \\
\hline Operating Temperature Range & Tone & & -40 & -10 to +60 & +85 & ${ }^{\circ} \mathrm{C}$ \\
\hline Storage Temperature Range & $T_{\text {sto }}$ & & -40 & & +85 & ${ }^{\circ} \mathrm{C}$ \\
\hline Shunt Capacitance & $\mathrm{C}_{0}$ & & & & 4 & $\mathrm{pF}$ \\
\hline Insertion Loss & $I_{L}$ & & & & 3 & $d B$ \\
\hline Motional Resistance & $R_{M}$ & & & & 50 & $\Omega$ \\
\hline Quality Factor (unloaded) & $Q_{u}$ & & 8,000 & & 20,000 & \\
\hline Aging (First year) & F. & at $25^{\circ} \mathrm{C}$ & -10 & & +10 & PPM \\
\hline
\end{tabular}

Mechanical Dimensions

D02(TO-39/3A) (mm)

D02(TO-39/3A) (inch)
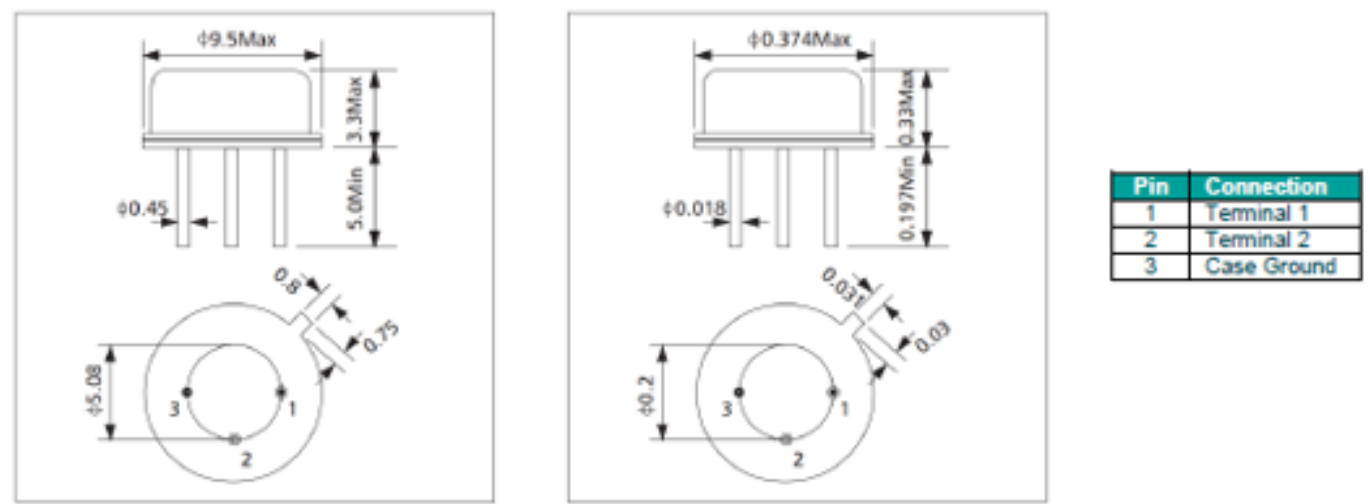